SKELETAL MUSCLE WEAKNESS AND MITOCHONDRIAL DYSFUNCTION IN THE OSTEOGENESIS IMPERFECTA MURINE (OIM) MODEL

A Dissertation
Presented to
The Faculty of the Graduate School
At the University of Missouri-Columbia
In Partial Fulfillment
of the Requirements for the Degree
Doctor of Philosophy
bictoria L Gremminger
Dr. Charlotte L. Phillips, Dissertation Advisor

May 2021 
The undersigned, appointed by the dean of the Graduate School, have examined the dissertation entitled

\section{SKELETAL MUSCLE WEAKNESS AND MITOCHONDRIAL DYSFUNCTION IN THE OSTEOGENESIS IMPERFECTA MURINE (OIM) MODEL}

presented by Victoria L Gremminger

a candidate for the degree of Doctor of Philosophy of Biochemistry, and hereby certify that, in their opinion, it is worthy of acceptance.

Dr. Charlotte Phillips

Dr. R. Scott Rector

Dr. Laura C. Sculz

Dr. Antje Heese

Dr. Michael Petris 


\section{DEDICATION}

I would like to dedicate this dissertation to my wonderful husband, Thomas Gremminger. I am beyond grateful for your continued love and support. You have been such a rock to me, providing constant encouragement throughout the progression of my dissertation. I could think of no one else I would have rather shared this journey with and cannot wait to see where our next adventure takes us. I would also like to dedicate this dissertation to my sweet baby, Gus, who has been patient with me as I have learned to navigate motherhood while finishing my degree and has brought so much joy into my life, during the sometimes challenging and difficult moments of finishing my dissertation research. 


\section{ACKNOWLEDGEMENTS}

I have been incredibly fortunate to have had so many individuals influencing and supporting me both personally and professionally as I have been working towards the completion of my dissertation. I am beyond grateful for the impact they have had in my life and could not have come this far without their support.

First, I would like to thank my family for always loving, supporting, and motivating me. My parents, Patty Brooks and Brett Grill, instilled a strong work ethic and sense of curiosity in me from a young age while facilitating my interest in learning, and for that, I am grateful. I would also like to thank my stepfather, William Brooks. I would especially like to express gratitude towards my mom, Patty Brooks, for always supporting and encouraging me to chase my dreams, teaching me to be an independent thinker, and always helping me find a way to overcome obstacles. I would like to thank my sisters, Samantha and Alexandria Grill, for always supporting me and distracting me during stressful times. I would like to acknowledge Pat, Dan, and Anna Gremminger for their continuous love, support, and interest in my research. I am also grateful to Madison Rhoades and Bilal Quadri for their friendship and our mutual love of science.

I would next like to thank all of the individuals who have contributed to my professional development, always helping me to improve as a scientist. First, I am grateful to my committee members Dr. R. Scott Rector, Dr. Laura C Schulz, Dr. Antje Heese, and Dr. Michael Petris. Their thoughtful comments and discussions significantly improved my research and taught me how to better think as a scientist especially in the way I interpret and present my data. I am especially grateful to Dr. Rector and Dr. Schulz 
for allowing me to work in their laboratories learning new technical skills to answer my research questions. Next, I would like to thank the numerous undergraduate researchers whom I have had the privilege of working with; specifically I would like to thank Salah Daghlas, Alp Kalvechi, Ashley Aguillard, and Emily Harrelson for their hard work and dedication to our research projects and for allowing me to develop my mentoring skills while working with them. I have thoroughly enjoyed watching each of them develop their own research questions and gaining confidence as independent researchers. I am also thankful to Dr. Youngjae Jeong for teaching me everything he knows about skeletal muscle in osteogenesis imperfecta. His constant patience and assistance at the beginning of my research was pertinent to my success in the laboratory. I would like to acknowledge Catherine Omosule for being a wonderful lab mate, and I am grateful for the friendship we have developed whilst completing our dissertations together.

Finally, I would like to thank my amazing advisor and mentor, Dr. Charlotte L Phillips. Dr. Phillips has always encouraged and supported me scientifically and personally, and her genuine interest in her students and concern for their well-being has significantly enhanced my graduate school experience. Her relentless patience and dedication to her students is inspiring and has been paramount to my success as a graduate student. I am thankful to her for always letting me pursue the research questions that interest me, helping me find the tools and individuals necessary to answer those questions, and for encouraging me to share my research, allowing me to present at numerous meetings over the past four years. She has been an amazing mentor and role model and I will be forever grateful that I had the privilege to be one of her graduate students. 


\section{TABLE OF CONTENTS}

ACKNOWLEDGEMENTS

LIST OF TABLES

vi

LIST OF FIGURES

vii

ABSTRACT

$\mathrm{X}$

CHAPTERS

I. INTRODUCTION AND REVIEW OF THE LITERATURE

$\begin{array}{ll}\text { Osteogenesis imperfecta } & 2\end{array}$

$\begin{array}{ll}\text { Bone biology } & 13\end{array}$

$\begin{array}{ll}\text { Skeletal muscle biology } & 16\end{array}$

$\begin{array}{ll}\text { Bone muscle crosstalk } & 25\end{array}$

Skeletal muscle pathology in disease $\quad 35$

Research Goals and Objectives $\quad 42$

II. COMPROMISED EXERCISE CAPACITY AND MITOCHONDRIAL DYSFUNCTION IN THE ONTOGENESIS IMPERFECTA MURINE (OIM) MOUSE MODEL

Abstract

Introduction

48

Methods

Results

Discussion

Supplementary material for Chapter II 
III. SKELETAL MUSCLE SPECIFIC MITOCHONDRIAL DYSFUNCTION AND ALTERED ENERGY METABOLISM IN A MURINE MODEL $($ OIM/OIM $)$ OF SEVERE OSTEOGENESIS IMPERFECTA

$\begin{array}{lc}\text { Abstract } & 93 \\ \text { Introduction } & 94 \\ \text { Methods } & 97 \\ \text { Results } & 102 \\ \text { Discussion } & 118 \\ \text { Supplementary material for Chapter III } & 124 \\ \text { IV. PERSPECTIVES AND FUTURE DIRECTIONS } & 129 \\ & \\ \text { BIBLIOGRAPHY } & 137 \\ \text { VITA } & 183\end{array}$




\section{LIST OF TABLES}

$\underline{\text { Table }}$

Page

I-1 Mutations leading to osteogenesis imperfecta and representative 4 mouse models

I-2 Studies highlighting the mechanical relationship of muscle and bone

I-3 Myokines and their roles on bone

I-4 Osteokines and their roles on muscle

II-S1 The swimming exercise regimen of wild-type and oim/oim mice was initiated at 6 weeks of age and completed at 4 months of age

II-S2 Femoral microarchitecture and biomechanical properties of male WT and oim/oim in-cage control, volunteer wheel running, and swimming exercised mice

II-S3 Femoral microarchitecture and biomechanical properties of female WT and oim/oim in-cage control, volunteer wheel running, and swimming exercised mice 


\section{LIST OF FIGURES}

Figure

$\begin{array}{lll}\text { I-1 Collagen synthesis } & 8\end{array}$

$\begin{array}{ll}\text { I-2 Sarcomere organization } & 20\end{array}$

I-3 Skeletal muscle and extracellular matrix organization 23

I-4 Osteocyte regulation of bone remodeling 30

$\begin{array}{ll}\text { I-5 Oxidative phosphorylation } & 40\end{array}$

II-1 Volunteer wheel running and swimming in male mice 60

$\begin{array}{lll}\text { II-2 } & \text { Femoral biomechanical properties } & 63\end{array}$

II-3 Electron transport chain complex protein content 66

$\begin{array}{lll}\text { II-4 Mitochondrial function } & 68\end{array}$

II-5 Protein markers of mitochondrial biogenesis and 70 mitophagy (males)

II-6 Protein markers of mitophagy in isolated mitochondria 71

II-7 Transmission electron microscopy mitochondrial analysis 73 in soleus muscle

II-8 Mitochondrial DNA to nuclear DNA ratio 74

II-9 Glycogen content in gastrocnemius muscle 75

II-S1 Volunteer wheel running and swimming in female mice 86

II-S2 Distance traveled by male and female oim/oim and WT mice 88

II-S3 Protein markers of mitochondrial biogenesis and $\quad 90$ mitophagy (female)

II-S4 Representative amido black staining used for western blots 91

III-1 Mitochondrial respiration and citrate synthase activity in 104 female gastrocnemius, liver, and heart isolated mitochondria 
III-2 Oxidation of $\left[1-{ }^{14} \mathrm{C}\right]$-palmitate by whole muscle and isolated 106 mitochondria

III-3 Myosin heavy chain immunohistochemical analysis 108

III-4 Hydrogen peroxide production in isolated mitochondria 109

$\begin{array}{lll}\text { III-5 Body composition } & 111\end{array}$

$\begin{array}{lll}\text { III-6 Energy expenditure } & 113\end{array}$

$\begin{array}{lll}\text { III-7 Glucose tolerance tests } & 115\end{array}$

III-8 Measurement of serum triglycerides and non-esterified 116 fatty acids

III-S1 Gastrocnemius and quadriceps respiration in 2-month old oim/oim 123 and WT mice

III-S2 Gastrocnemius mitochondrial respiration in heterozygous 124 oim mice

III-S3 Activity levels in oim/oim and WT mice 125

III-S4 Food and water consumption in oim/oim and WT mice 126

III-S5 Oxygen consumption and carbon dioxide production in 127 oim/oim and WT mice 


\title{
SKELETAL MUSCLE WEAKNESS AND MITOCHONDRIAL DYSFUNCTION IN THE OSTEOGENESIS IMPERFECTA MURINE (OIM) MODEL
}

\author{
Victoria L Gremminger
}

\author{
Dr. Charlotte L. Phillips, Dissertation Advisor
}

\begin{abstract}
Osteogenesis imperfecta (OI), also commonly referred to as brittle bone disease, is a heritable connective tissue disorder occurring in roughly 1:15,000 births. OI arises as a result of mutations in the type I collagen genes, COL1A1 and COL1A2, approximately $85 \%$ of the time with the remaining $15 \%$ of cases arising from mutations in genes involved in posttranslational modification of type I collagen, osteoblast maturation or mineralization. OI is a heterogeneous disorder that can be classified into four major types with severity ranging from perinatal lethality to premature osteoporosis. As the name suggests, osteogenesis imperfecta, literally translating to imperfect bone formation, results in bone fragility with patients often experiencing many fractures throughout their lifetime. While bone fragility is the most prominent manifestation of OI, skeletal muscle weakness, cardiopulmonary complications, short stature, and craniofacial abnormalities are also common. There is currently no cure for OI and therapeutic options rely on mitigating symptoms, primarily through the use of bone anti-resorptive agents referred to as bisphosphonates. Although, current treatment options focus solely on bone health, skeletal muscle weakness is a common manifestation in OI, where $80 \%$ of patients with mild OI experience muscle force deficits, and with even higher percentages in patients with more clinically severe OI. Historically, OI muscle weakness was largely attributed to inactivity with recent studies highlighting its inherent nature in both patients and
\end{abstract}


mouse models. Studies investigating the mechanisms by which skeletal muscle weakness arises in OI are limited, despite the large prevalence.

My research sought to better understand OI muscle weakness primarily through the investigation of mitochondrial health in a mouse modeling a severe human type III OI (oim/oim), as mitochondria are important regulators of energy metabolism and overall cell health. We hypothesized that oim/oim mice, exhibiting severe skeletal muscle weakness would exhibit mitochondrial dysfunction suggesting a correlation between skeletal muscle and mitochondrial function. To test this hypothesis, we assessed mitochondrial function and content in the oim/oim mouse. One of our major findings was the observation that oim/oim mice exhibit $>50 \%$ reductions in gastrocnemius mitochondrial respiration rates relative to wildtype littermates. Additionally, we found that citrate synthase activity in oim/oim isolated gastrocnemius mitochondria was reduced relative to wildtype littermates. Furthermore, to determine if skeletal muscle mitochondrial function correlated with skeletal muscle severity, we evaluated mitochondrial respiration in a mouse model of mild OI (+/oim). We did not find differences between +/oim and WT gastrocnemius mitochondrial respiration suggesting that mitochondrial function does correlate with skeletal muscle function. Moreover, we did not observe changes in mitochondrial respiration in oim/oim liver and heart suggesting the mitochondrial dysfunction is not global in the oim/oim mouse.

Additionally, we sought to investigate whole body metabolic alterations, as skeletal muscle comprises roughly $50 \%$ of body mass and is a significant contributor to the body's resting metabolic rate. We hypothesized that skeletal muscle mitochondrial dysfunction in the oim/oim mouse would lead to changes in metabolic parameters 
including altered substrate utilization, altered body composition, and changes in energy expenditure. Interestingly, we did not observe changes in substrate utilization, although we did note increased energy expenditure and subtle changes in body composition with oim/oim animals exhibiting reduced percentages of fat mass and increased percentages of lean mass relative to wildtype littermates.

Overall, my research was the first to implicate mitochondrial dysfunction in the pathophysiology of OI using a mouse model of severe OI. This work has led to numerous studies in other mouse models evaluating mitochondrial function and energy metabolism. While there is more work to be done to further understand the mechanisms and correlation between mitochondrial dysfunction and skeletal muscle weakness in OI, this novel finding has initiated a new area of research in OI and has contributed to the overall understanding of OI muscle weakness. 


\section{CHAPTER I}

INTRODUCTION AND REVIEW OF THE LITERATURE 


\section{$\underline{\text { Osteogenesis imperfecta }}$}

Osteogenesis imperfecta (OI), also commonly referred to as "brittle bone disease," is a genetically and clinically heterogeneous heritable connective tissue disorder affecting roughly $1: 15,000$ births[1,2]. While the most striking feature of the disease is bone fragility and skeletal dysplasia, more recent research has implicated inherent muscle weakness as part of the pathophysiology of the disease[3-6] and a small number of studies have highlighted the presence of a metabolic phenotype in OI $[7,8]$. Other manifestations include short stature, cardiopulmonary abnormalities, kyphoscoliosis, and the presence blue-gray sclera[1,2].

The heterogeneity of OI is exemplified by the more than 1500 recognized disease-causing mutations $[9,10]$. Roughly $85 \%$ of the time OI is the result of qualitative (dominant negative) or quantitative (haplo-insufficient) defects in type I collagen due to mutations in the type I collagen genes: COL1A1 and COL1A2[1,2,9,11]. The remaining $15 \%$ of cases are the result of mutations in genes implicated in bone mineralization, posttranslational modifications, folding, and secretion of type I collagen, as well as those involved in osteoblast maturation and function[1,2,12].

As might be expected from the vast number of disease-causing mutations affecting multiple genes exhibiting unique functions, the clinical severity of the disease ranges considerably, from asymptomatic to perinatal lethality[1,2,12,13]. Classically, there are four subtypes of OI (Sillence classification; I-IV) with type I being the most mild form of the disease, type II being the most severe (resulting in perinatal lethality), type III being the most severe viable form (often resulting in the non-ambulation of patients), and type 
IV being of moderate severity [13]. As the genetic understanding and the number of identified ,novel OI-causing mutations has expanded, there has been a shift in the classification of OI, with 20 types currently recognized in the Online Mendelian Inheritance in Man (OMIM) database[12,14]. A summary of known genetic loci responsible for human OI with corresponding mouse models can be seen in Table I-1. 
Table I-1: Summary of known osteogenesis imperfecta genetic loci and representative mouse models.

\begin{tabular}{|c|c|c|c|}
\hline Gene Symbol & \begin{tabular}{|l} 
Sillence \\
Type
\end{tabular} & Protein & Mouse Models (OI type) \\
\hline \multicolumn{4}{|c|}{$\begin{array}{l}\text { Group A: Autosomal Dominant and defects in collagen } \\
\text { synthesis, structure, or processing }\end{array}$} \\
\hline $\begin{array}{l}\text { COL1A1 or } \\
\text { COL1A2 }\end{array}$ & $\begin{array}{l}\text { I, II, III, } \\
\text { and IV }\end{array}$ & $\begin{array}{l}\text { Collagen type I, alpha1 or } \\
\text { alpha2 }\end{array}$ & $\begin{array}{l}\text { COL1A1 Mutations: } \\
\text { BrtlIV/+ (type IV), Jrt/+ } \\
\text { (type IV/III) COL1A2 } \\
\text { Mutations: oim/oim (type } \\
\text { III), +/oim (type I), +/G610C } \\
\text { (type IV) }\end{array}$ \\
\hline \multicolumn{4}{|c|}{ Group B: Defects in collagen post translational modification } \\
\hline CRTAP & VII & Cartilage-associated protein & $\operatorname{Crtap}^{-/-}$(type VII) \\
\hline LEPRE1/P3H1 & VIII & $\begin{array}{l}\text { Leucine proline-enriched } \\
\text { proteoglycan1/prolyl 3- } \\
\text { hydroxylase-1 }\end{array}$ & $P 3 H 1^{--}($type VIII) \\
\hline PPIB & IX & $\begin{array}{l}\text { Peptidylprolyl isomerase } \\
\text { B/cyclophilin B }\end{array}$ & $P p i b^{--}$(type IX) \\
\hline TMEM38B & XIV & Transmembrane protein $38 \mathrm{~B}$ & Tric- $b$ (type XIV) \\
\hline \multicolumn{4}{|c|}{ Group C: Defects in collagen folding and cross linking process } \\
\hline SERPINH1 & $\mathrm{X}$ & $\begin{array}{l}\text { Serpin Peptidase inhibitor, } \\
\text { clade } H \text {, member } 1 / \text { heat } \\
\text { shock protein } 47\end{array}$ & $H s p 47^{--}$(type X) \\
\hline FKBP10 & $\mathrm{XI}$ & FK506 binding protein 65 & $F k b p 10^{-/}($type XI) \\
\hline \multicolumn{4}{|c|}{ Group D: Defects in bone mineralization } \\
\hline IFITM5 & $\mathrm{V}$ & $\begin{array}{l}\text { Interferon-induced } \\
\text { transmembrane protein } 5\end{array}$ & Ifitm $5^{d}$ (type V) \\
\hline SERPINF1 & VI & $\begin{array}{l}\text { Pigment epithelium-derived } \\
\text { factor }\end{array}$ & Pedf $^{\prime}$ (type VI) \\
\hline \multicolumn{4}{|c|}{ Group E: Defects in osteoblast development and function } \\
\hline SP7 & XII & $\begin{array}{l}\text { Transcription factor } \\
\text { 7/osterix }\end{array}$ & $S P 7^{-1}(O s x)($ type XIII) \\
\hline WNT1 & $\mathrm{XV}$ & $\begin{array}{l}\text { Wingless-type MMTV } \\
\text { integration site family, } \\
\text { member } 1\end{array}$ & Wnt1 ${ }^{\text {SW }}$ (type XV) \\
\hline CREB3L1 & XVI & $\begin{array}{l}\text { cAMP responsive element } \\
\text { binding protein } 3 \text { like } 1\end{array}$ & OASIS $^{-/-}($type XVI) \\
\hline
\end{tabular}


The genetic and clinically heterogeneous nature of OI presents a challenge when it comes to treatment strategies. The diversity in OI mutations and severities prevents use of a "one size fits all" approach; instead physicians focus on mitigating symptoms, as there is currently no cure for OI $[2,12]$. Current treatments for OI are predominately focused on mitigating the bone fragility, with little to no consideration of muscle weakness. These include the use of bone antiresorptive and osteoanabolic pharmaceutical compounds, physical therapy, and surgical interventions such as intermedullary rodding in pediatric patients[2,12,15-18].

The current standard of treatment in OI includes the administration of intravenous or oral bone antiresorptive bisphosphonate therapies, which improve bone mineral density (BMD) although their efficacy in fracture reduction remains debated and the safety and efficacy in pediatric patients remains unclear [11,12,16,19]. Additionally, long term use of bisphosphonates may have an overall detrimental effect on bone due to decreased bone remodeling, increased accumulation of microfractures thus compromising the bone integrity, and potential association with osteonecrosis of the jaw[11,15,19-21]. The use of bone anabolic therapies, including parathyroid hormone, anti-sclerostin, and antiTGF $\beta$, are currently being evaluated for use in OI[15,22-24]. It is important to note that while all these compounds increase bone formation, they do not alter the compromised integrity of bone material due to type I collagen defects.

\section{Type I collagen structure and synthesis}

There are 28 members of the collagen superfamily, making collagen the most abundant protein in the body, comprising roughly $30 \%$ of total protein mass [25]. Type I collagen, 
found throughout the body in various connective tissues and accounting for approximately $95 \%$ of bone collagen content, is a fibrillar type of collagen organized as a heterotrimer composed of $\alpha 1(\mathrm{I})_{2} \alpha 2(\mathrm{I})$ chains [26]. Each of these alpha chains exists as a polyproline II-like helix, which supercoils to form a triple helical motif $300 \mathrm{~nm}$ in length and $1.5 \mathrm{~nm}$ in diameter [26,27]. This process is facilitated by the repeating primary amino acid sequence Gly-X-Y, where $\mathrm{X}$ and $\mathrm{Y}$ are often proline and hydroxyproline, respectively. The presence of glycine at every third amino acid position allows for the tight packing of the triple helix, as glycine lacks a side chain, and can be buried within the helix [28].

The biosynthesis of collagen (Figure I-1) is complex and requires numerous temporally and spatially coordinated processes including many post translational modifications (PTMs)[26,29-31]. The initial step of type I collagen biosynthesis is the transcription of COL1A1 and COL1A2 mRNA. Once transcribed, the mRNA enters the cytoplasm where it is translated on the ribosomes of rough endoplasmic reticulum (ER) in the $\mathrm{N}$ - to Cterminal direction. The newly translated molecule, referred to as a pre-pro-peptide, contains a signal on the $\mathrm{N}$-terminus directing it to the $\mathrm{ER}$ where it is recognized by a signal recognition particle [26,29-31]. Once inside the ER lumen, three main events take place signifying the transition to procollagen: 1) the signal on the N-terminus is cleaved, 2) select lysine and proline amino acids are hydroxylated and 3) hydroxylysine is glycosylated via the addition of monosaccharides[26,29-31]. This hydroxylation and glycosylation contribute to the overall thermal and mechanical stability of type I collagen[26,29-31]. Following the PTM of the pro- $\alpha$ chains, the alignment of three pro- $\alpha$ chains, facilitated by disulfide bonding of cysteine residues in the C-telo-peptide, occurs 
leading to triple helix formation in a zipper-like fashion from $\mathrm{C}$ - to $\mathrm{N}$-terminus, a process that on average takes an astounding 14 minutes[26,29-31]. Once the triple helix is formed, the chaperone protein hsp47 binds to and increases stability of the triple helix while preventing premature procollagen aggregates as procollagen moves to the golgi[26,29-31]. From the golgi, procollagen is transported to the extracellular space, via secretory vesicles, where the globular N-and C- pro-peptides are cleaved [the rate limiting step of fibrillogenesis] [32]. Following cleavage of the pro-peptides, lysyl oxidase catalyzes the formation of reactive aldehydes on lysine and hydroxylysine residues which will spontaneously react to form intramolecular crosslinks[29]. 


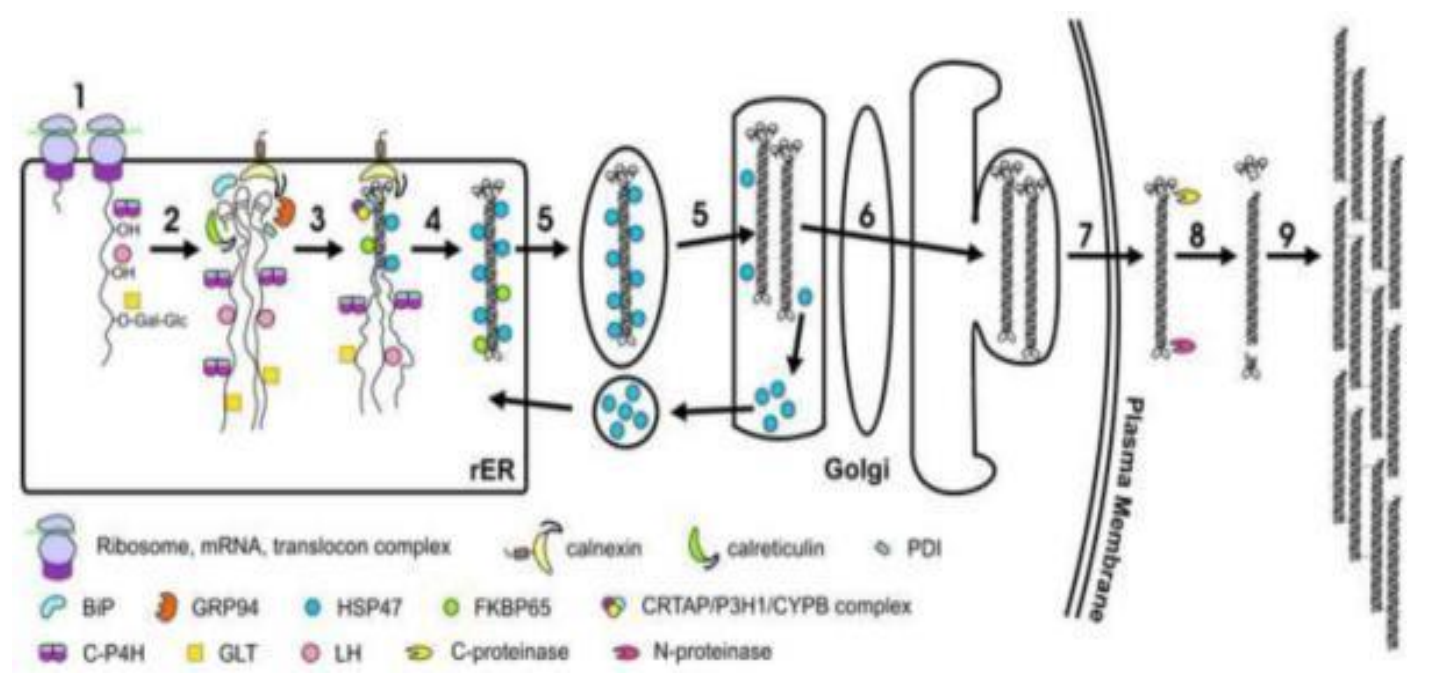

Figure I-1 Collagen Synthesis. Originally published in Trends in Cell Biology (Makareeva et al., 2011)[33] 1) Cotranslational translocation of procollagen chains into the lumen of rough ER. 2) C-propeptide domains of two pro- $\alpha 1$ (I) and one pro- $\alpha 2$ (I) chains assemble into a trimer and fold. 3) The triple helix is nucleated at the $\mathrm{C}$-terminal end. 4) The triple helix propagates towards the $\mathrm{N}$ terminal end. 5) Procollagen is cotransported with HSP47 to the cis-Golgi network following N-propeptide folding. 6) Procollagen is transported through the Golgi stack. 7) Procollagen is secreted into the extracellular space. 8) N- and Cpropeptides are cleaved via extracellular $\mathrm{N}$ - and $\mathrm{C}$ - proteinases. 9) Mature collagen assembles into fibers. 
Bone derives its mechanical properties and strength in part from its mineral and organic components, hydroxyapatite and collagen fibrils, respectively [26,29,34]. The collagen matrix has been demonstrated to contribute mainly to bone toughness and strength whereas the mineral component contributes predominately to bone stiffness [35]. Type I collagen fibrils themselves exhibit great tensile strength that is "gram for gram" stronger than steel allowing them to withstand great forces [31], as evidenced by tendon, where type I collagen is abundant, connecting bone and muscle while enduring large forces generated by muscle pull [31].

That osteogenesis imperfecta is caused most often by mutations in the type I collagen genes exemplifies the significance of type I collagen strength and organization for overall bone biomechanics, demonstrating that qualitative and/or quantitative defects in type I collagen severely compromise bone $[1,26]$. For example, the osteogenesis imperfecta murine (oim) model, containing a mutation in the Colla2 gene, has been used to analyze the role of intrinsic bone properties on bone. Bone biomechanical properties of oim mice demonstrate reduced biomechanical strength, evidenced by a $60 \%$ reduction in post-yield deformation, a parameter often associated with accumulated microdamage, viscoelastic response, and plastic deformation, ultimately indicating a weaker bone as the result of the Colla2 mutation [26,36-38].

\section{oim mouse model}

The osteogenesis imperfecta murine (oim) mouse model contains a spontaneous deletion of the $3983^{\text {rd }}$ nucleotide in the Colla2 gene[39]. This mutation results in the production of homotrimeric type I collagen $\left[\alpha 1(\mathrm{I})_{3}\right]$ rather than the normal heterotrimeric type I 
collagen $\left[\alpha 1(\mathrm{I})_{2} \alpha 2(\mathrm{I})\right][39]$. The homozygous oim/oim mouse models a patient with severe type III OI [40,41], exhibiting severe skeletal fragility, whereas the heterozygous +/oim mouse models more mild type I OI, exhibiting less severe skeletal fragility [39].

Bone fragility in the oim mouse can be at least partially explained by the reduced ability of homotrimeric collagen to resist tensile stress in the oim mouse as compared to wildtype collagen[42]. Another contribution to the skeletal fragility noted in the oim/oim mouse is likely the reduced ability to form "normal" mineral crystals relative to wildtype animals, a result of homotrimeric collagen altering the scaffold on which this mineral is laid[43]. Numerous studies have been conducted on the oim mouse, leading to a better understanding of the contribution of type I collagen to overall bone biomechanical properties including biomechanical strength[36,37,42,44,45].

In addition to the severe bone fragility, the oim/oim mice exhibit severe muscle weakness and studies in the oim mouse were the first to implicate inherent skeletal muscle weakness as part of the disease pathology of OI[3]. Gentry et al. demonstrated that oim/oim skeletal muscles had reduced specific contractile generating force, [contractile generating force normalized to myofiber cross sectional area] in the gastrocnemius, tibialis anterior, and plantaris muscles in addition to reduced body weights, muscle weights, and relative muscle weights[3]. This study launched the field of muscle research in osteogenesis imperfecta. 


\section{Skeletal muscle weakness and energy metabolism in OI}

Historically, muscle weakness in osteogenesis imperfecta had been attributed to hypoactivity, with patients reporting fatigue and reduced exercise capacity in addition to muscle weakness[6,46]. Studies in the oim/oim mouse were the first to demonstrate an inherent muscle pathology as part of the pathophysiology of OI[3]. Following this novel discovery, clinicians and basic scientists began to consider and investigate muscle function in OI patients and other mouse models of OI. Of these studies, one of the most significant findings was that $80 \%$ of patients with a type I OI phenotype caused by a mutation in either COL1A1 or COL1A2 experienced muscle force deficits when muscle force was assessed via mechanography[6,47]. Muscle function correlates with OI severity, with muscle force deficits often more severe in patients with moderate and severe types IV and III OI, respectively [48]. Another interesting finding by PouliotLaforte et al., was that children with OI who experienced muscle force deficits were just as active as their healthy peers, although neither group of children met the daily recommended amount of activity, suggesting that reduced physical activity is not the leading cause of muscle weakness in children with OI[47]. Over the past decade, efforts to better characterize and understand muscle weakness in patients with OI have drastically increased. However, the cause of muscle weakness in OI remains unknown, although numerous mechanisms continue to be investigated including the role of altered collagen in muscle connective tissue layers and tendon, reduced mobility in patients with OI, and altered signal transduction and mechanotransduction between muscle and bone[2,14,48]. 
In addition to patient studies, skeletal muscle function has also been assessed in several mouse models of OI, providing useful insights to potential mechanisms of muscle weakness, as well as for evaluation of novel therapeutic options targeting muscle weakness in OI[3,14,49]. These studies have demonstrated a positive correlation between OI severity and skeletal muscle weakness. Skeletal muscle weakness is absent in the +/G610C or "Amish" mouse model of mild OI, containing a glycine to cysteine substitution in the triple helical domain; a mutation also found in an old Amish kindred affected by OI[49,50]. In contrast, in models of severe OI such as the oim/oim and Collal $^{\mathrm{Jrt} / \mathrm{+}}$, reduced activity and skeletal muscle weakness are observed[3,8,51,52]. The idea that skeletal muscle weakness may be mutation- and severity- specific in OI warrants further investigation to better understand the mechanisms of this muscle weakness.

The Collal ${ }^{J t t /+}$ is the first combined model of both severe type IV OI and Ehlers Danlos Syndrome (EDS) due to a COL1A1 mutation leading to skipping of exon 9 and subsequent 18 amino acid deletion in the triple helical domain[51]. EDS is a genetic connective tissue disorder arising primarily due to mutations in type $\mathrm{V}$ collagen, resulting in skin hyper-elasticity, joint hypermobility, skin fragility, and blood vessel fragility, although a subset arise from mutations near the amino terminal end of type I collagen $[53,54]$. In addition to reduced physical activity and skeletal muscle weakness, recent evidence in the Collal ${ }^{J t t / 4}$ mouse suggests a metabolic phenotype exists and contributes to the pathophysiology of the disease[8]. While there are limited studies evaluating energy metabolism in OI, Cropp and Myers, in 1972, described the presence of a hypermetabolic state in adolescent male OI patients[7]. Taken together, the report of a 
metabolic phenotype in both the mentioned patient cohort and in the CollalJrt/+ model suggest energy metabolism as an important contributor to the pathophysiology of OI. The idea of altered energy metabolism in OI may not be that surprising due to the wide prevalence of skeletal muscle weakness in patients with OI and the fact that skeletal muscles account for roughly $20 \%$ of the basal metabolic rate[15,48,55,56].

While the bone phenotype in OI has been well characterized and thoroughly evaluated in both patients and countless animal models, skeletal muscle weakness in OI is a relatively recent finding, and a thorough understanding of the mechanisms leading to this phenomenon remain unknown. However, understandings of these mechanisms should be pursued to identify novel therapeutic targets that will not only improve the observed muscle phenotype, but also to ultimately improve bone strength as bones and muscles are highly synergistic tissues that communicate extensively with one another[57-61].

\section{Bone Biology}

Bone is a highly metabolic and dynamic endocrine tissue that has major roles in locomotion, calcium and phosphate storage, and energy metabolism[62-64]. Bone can be divided into a mineral phase and an organic phase consisting of primarily hydroxyapatite and type I collagen, respectively and is composed of four major cell types: osteoblasts, osteoclasts, osteocytes, and bone lining cells $[62,63]$. These four cell types facilitate the extensive remodeling necessary for the skeletal adaptation to mechanical strain, fracture repair, and calcium homeostasis[63,64].

Osteoclasts control the first step of the bone remodeling process; reabsorption of bone. Osteoclasts are multinucleated cells coming from a macrophage/hematopoietic lineage, 
For bone resorption to occur, there must be proliferation of the osteoclast precursor cells and differentiation of these cells into mature osteoclasts, a process referred to as osteoclastogenesis $[65,66]$. Osteoclasts then adhere to the cell surface where they will break down the mineral hydroxyapatite component of bone followed by the degradation of the organic collagen matrix, a process facilitated by the secretion of multiple factors including tartrate resistant alkaline phosphatase or TRAP $[65,66]$. Osteoclast differentiation and activity are regulated by two primary essential factors: macrophage colony stimulating factor (M-CSF) and receptor for activation of nuclear factor kappa B ligand (RANKL)[66]. The process of bone resorption is also highly regulated by the osteoblast and cells from the osteoblast lineage including pre-osteoblasts, osteocytes, and bone-lining cells[67].

Osteoblasts, deriving from the mesenchymal stem cell lineage, facilitate the formation of bone in the remodeling process[63,64,67]. Following bone resorption, osteoblasts are recruited to the resorption site where they will deposit new osteoid onto the bone matrix[63]. This process is largely enabled by expression of Runx2, a master regulator of osteoblast differentiation, and canonical WNT signaling[63]. One gene upregulated by Runx 2 is COL1A1, resulting in production of type I collagen, which will be secreted by the osteoblast[63,68]. Expression of COL1A1 in addition to Runx 2 signifies the transition of pre-osteoblast to mature osteoblast [63]. In addition to their significant role in bone formation, osteoblasts are also essential regulators of osteoclasts and thus, bone resorption $[63,64]$. The best characterization of this regulation is observed via osteoblasts production of osteoprotegerin (OPG), a decoy receptor for RANKL, which results in 
inhibition of osteoclast differentiation[67]. Other cells of the osteoblastic lineage also play important roles regulating the tightly coordinated bone remodeling process.

Osteocytes and bone lining cells both arise from mature osteoblasts and are key players in bone remodeling[63]. The function of bone lining cells has not been well characterized and little is known about these quiescent cells other than they tend to physically separate the osteoclast from the bone matrix[63]. Upon initiation of bone remodeling, these cells retract, allowing for bone resorption to begin[68]. Osteocytes also arise from osteoblasts, comprise up to $95 \%$ of all bone cells, and are known for their roles in orchestrating bone remodeling via control of osteoblasts and osteoclast, as well as in mechano-sensation[69]. Osteocytes are embedded in the bone matrix and have an average half-life of 25 years. Osteocytes possess dendritic processes which aid in communication with other osteocytes, osteoblasts, and osteoclasts[69,70].

Bone remodeling is a highly coordinated and tightly regulated process facilitated by the different bone cells. Due to the extensive remodeling that occurs in bone, it may not come as a surprise that alterations in the molecular pathways underlying these processes can result in bone disease such as osteogenesis imperfecta. As previously stated, $\sim 85 \%$ of OI cases arise due to mutations in type I collagen with the remaining cases arising due to mutations in collagen modification and folding, osteoblast differentiation and function, and bone mineralization; all of which are essential to bone remodeling[15] (Table I-1). These mutations lead to changes in several important signaling pathways regulating bone remodeling including the WNT and RANK/RANKL pathways, ultimately resulting in uncoordinated bone remodeling[15]. 


\section{$\underline{\text { Skeletal Muscle Biology }}$}

With the presence of over 600 muscles, skeletal muscle is the largest organ in the human body, comprising roughly $40-50 \%$ of body mass[71-74]. Skeletal muscle participates in many roles including body mechanics, energy metabolism and serves as a secretory organ[71-73]. Here, important aspects of skeletal muscle biology, including development and growth, structure and organization, and mechanical function, will be discussed.

\section{Embryonic Development}

Skeletal muscle arises from the paraxial mesoderm that gives rise to somites that eventually diverge into sclerotome and the dermomyotome, giving rise to bone/cartilage and skin/skeletal muscle, respectively[74,75]. Skeletal muscle formation progresses in five major steps including: delamination, migration, proliferation, determination, and differentiation[75]. Cells that give rise to skeletal muscle delaminate from the dermomyotome and migrate to the limb bud, processes facilitated by the factors Pax3, cmet, hepatocyte growth factor (HGF), and Lbx1[75,76]. Once myogenic precursors reach the limb bud, they begin expressing MyoD and Myf5, signifying the commitment of the cells to the myoblast lineage[77]. Evidence suggests that proliferation of myoblast precursor cells occurs before and after determination and is likely facilitated by Pax3 and c-met[77]. Differentiation occurs via the expression of MRF4 and myogenin, which together with MyoD and Myf5 make up the myogenic regulatory transcription factor family (MRFs)[76,77].

Following the final step of differentiation, myoblasts begin to express myosin heavy chains as part of terminal differentiation, at which point myoblasts will fuse to form 
multinucleated myotubes[76]. These myotubes will then assemble to form muscle fibers leading to the formation of specific muscle groups[76,77].

Skeletal muscle consists of multinucleated muscle fibers that can be broadly classified as either fast/white/glycolytic or slow/red/oxidative[78]. The expression of different myosin heavy chains is what distinguishes muscle fiber types and their cellular energy metabolism, although skeletal muscles are highly plastic allowing fibers to adapt to the muscles' activity level and needs[79]. There are four primary adult fiber types (MyHC$\beta /$ slow, MyHC-2A, MyHC-2B, and MyHC-2X) and two developmental types (MyHCemb and MyHC-neo) in skeletal muscle[78]. Type 1, or slow, fibers are red in appearance, have a high oxidative capacity, are slower to fatigue, and have relatively high mitochondrial volume density (2-3 fold higher compared to fast fibers)[80]. Type 2 or fast fibers are white in appearance, have a lower oxidative capacity, fatigue quickly, and have relatively lower mitochondrial volume density[80]. Type 2 fibers can be further broken down to the types $2 \mathrm{~A}, 2 \mathrm{~B}$, and $2 \mathrm{X}$ based on their oxidative capacities as fast oxidative, fast glycolytic, and fast intermediate; respectively[78].

In the mouse, all skeletal muscles express the embryonic (MyHC-emb) and slow (MyHC$\beta /$ slow) myosin heavy chains prior to embryonic day 16 (E16), at which point some fibers will begin to lose their MyHC- $\beta$ /slow expression, which is replaced by MyHC-neo, giving rise to two distinct populations of fibers expressing either MyHC-emb/MyHC-neo or MyHC-emb/MyHC- $\beta /$ slow[78]. The fibers expressing MyHC-emb/MyHC-neo or MyHC-emb/MyHC- $\beta$ /slow will develop into fast or slow fiber types, respectively[78]. 


\section{Muscle growth and regeneration}

While myoblasts arise from the mesenchymal stem cells during development, post-natal muscle growth and repair is facilitated by a population of stem cells referred to as satellite cells. These quiescent cells appear in limbs at embryonic day 17.5 arising from cells of the dermomyotome, similar to myoblasts[81,82]. Satellite cells lie between the basal lamina and the sarcolemma of each myofiber, and are heterogeneous in terms of cell number and Pax7 expression [83-85]. Although satellite cells are able to self-renew, the total number of satellite cells has been shown to decrease overtime with age as the number of myonuclei increase[81,82,86].

Due to the postmitotic nature of muscle fibers, they are not able to divide. They rely on satellite cells to provide the myonuclei required for maintenance of muscle by normal regeneration, hypertrophy, and repair[84]. Satellite cells are identified by their expression of Pax7, an important transcription factor in myogenesis and the canonical satellite cell biomarker [82,84]. Once satellite cells are activated, in response to an environmental signal or damage, they begin to express muscle regulatory factors (MRFs) which are also

present during development, including MyoD1, Myf5, and myogenin [81,84]. The activation, differentiation, and migration of these satellite cells ultimately lead to the cells fusing with one another to generate new myofibers or with a damaged muscle fiber for repair [81]. While there are many signals and factors regulating satellite cell function, three stand out that maybe relevant in OI, including WNT/ $\beta$-catenin signaling, NOTCH signaling, and extracellular matrix composition and stiffness[81].However, the relationship of these factors to OI satellite cell function has not yet been characterized. 


\section{Structure and organization}

The basic functional unit of skeletal muscle, a sarcomere, consists of the region from one Z-disc to another, as shown in figure I-2. The sarcomere is composed of thin actin and thick myosin filaments that facilitate muscle contraction. The thin actin filaments (Factin) are composed of actin monomers (G-actin) that exhibit polarity within the sarcomere. Additionally, thin actin filaments contain the proteins troponin and tropomyosin, important regulators of muscle contraction [87]. The thick myosin filament is composed of myosin II, a hexameric protein composed of four light chains and two heavy chains containing a rod and head region[87]. The two globular heads also serve as important facilitators of contraction[87].

Upon visualization of the sarcomere via electron micrograph (Figure I-2), several conserved and obvious structures stand out. The dark banded $\mathrm{Z}$ disc or $\mathrm{Z}$ band can be seen at either end of the sarcomere adjacent to the light isotropic or I band consisting of thin actin filaments[88]. Between the I-bands lies another dark banded region referred to as the anisotropic or A-band consisting of the thick myosin filaments[88]. In the very center of the sarcomere exists the M-band composed of proteins of the myomesin family serving as cross linkers between the thick myosin filaments and contributing to the hexagonal lattice within the A-band [88-90]. On either the side of the M-band a faint light line can be seen, a result of the absence of myosin heads on this region of the thick filament[89]. The area between these light bands within the A-band is referred to as the H-zone which shortens during contraction[34,88]. 


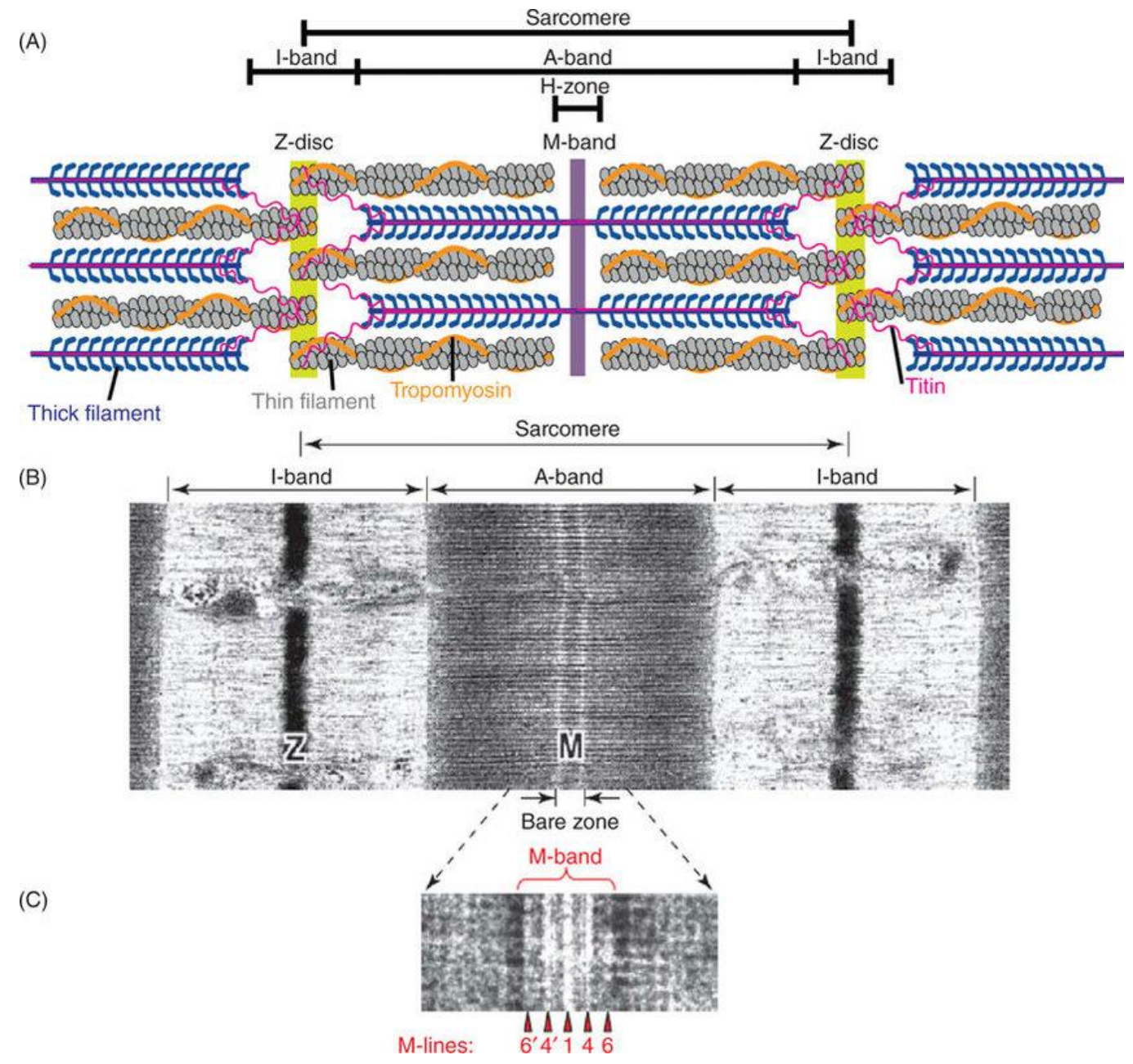

Figure I-2 Sarcomere organization. Originally published in Comprehensive Physilogy (Henderson et al., 2017)[91]. A) Schematic of sarcomere organization. B) Electron micrograph image of a sarcomere. C) Electron micrograph of the $\mathrm{M}$ zone of the sarcomere. 
As previously described, the basic structural and functional unit of a muscle is referred to as a sarcomere and is composed of thick myosin and thin actin filaments. Together these myofilaments make up the myofibril. Bundles of myofibrils are surrounded by a plasma membrane denoted as the sarcolemma and make up a muscle fiber. Skeletal muscles possess a highly organized extracellular matrix composed of a hierarchical arrangement of endo-, peri-, and epimysium (Figure I-3)[92]. The endomysium surrounds bundles of muscle fibers referred to as fascicles and bundles of fascicles surrounded by the perimysium make up skeletal muscle which is covered by the epimysium[92]. At the base of a skeletal muscle the epimysium connective tissue converges to the tendon which connects the muscle to bone[93]. The composition of collagen within these various extracellular components differ greatly. Type I collagen is most abundant in both the tendon and perimysium of the skeletal muscle while types I and III collagens are equally abundant in both the epi- and endomysium[92]. Interestingly, the organization of type I collagen differs from tendon and perimysium with the type I collagen in tendon being much more organized, aligning with the muscle axis, than in the perimysium which is likely a result from the different functions of each component [92]. This highly organized hierarchical extracellular matrix surrounding the muscle fibers is believed to help facilitate force transduction with all of the different levels believed to be interconnected mechanically[92,94]. In addition to the collagens found in these extracellular matrix components, type IV collagen networks are highly present in the basement membranes of skeletal muscles with Types VI, XV, and XVIII also present in smaller amounts[92]. Furthermore, while the endomysium and basement membrane are separate entities, they are interconnected with the basement membrane likely playing an important role in force 
transduction[92]. In addition to collagen which comprises roughly 1-10\% of dry muscle weight $[92,95,96]$, the extracellular matrix consists largely of proteoglycans (such as decorin), glycosaminoglycans, and glycoproteins (including integrins, fibronectin, and laminins) all interacting with one another to facilitate force transduction throughout the ECM of the skeletal muscle[92]. 


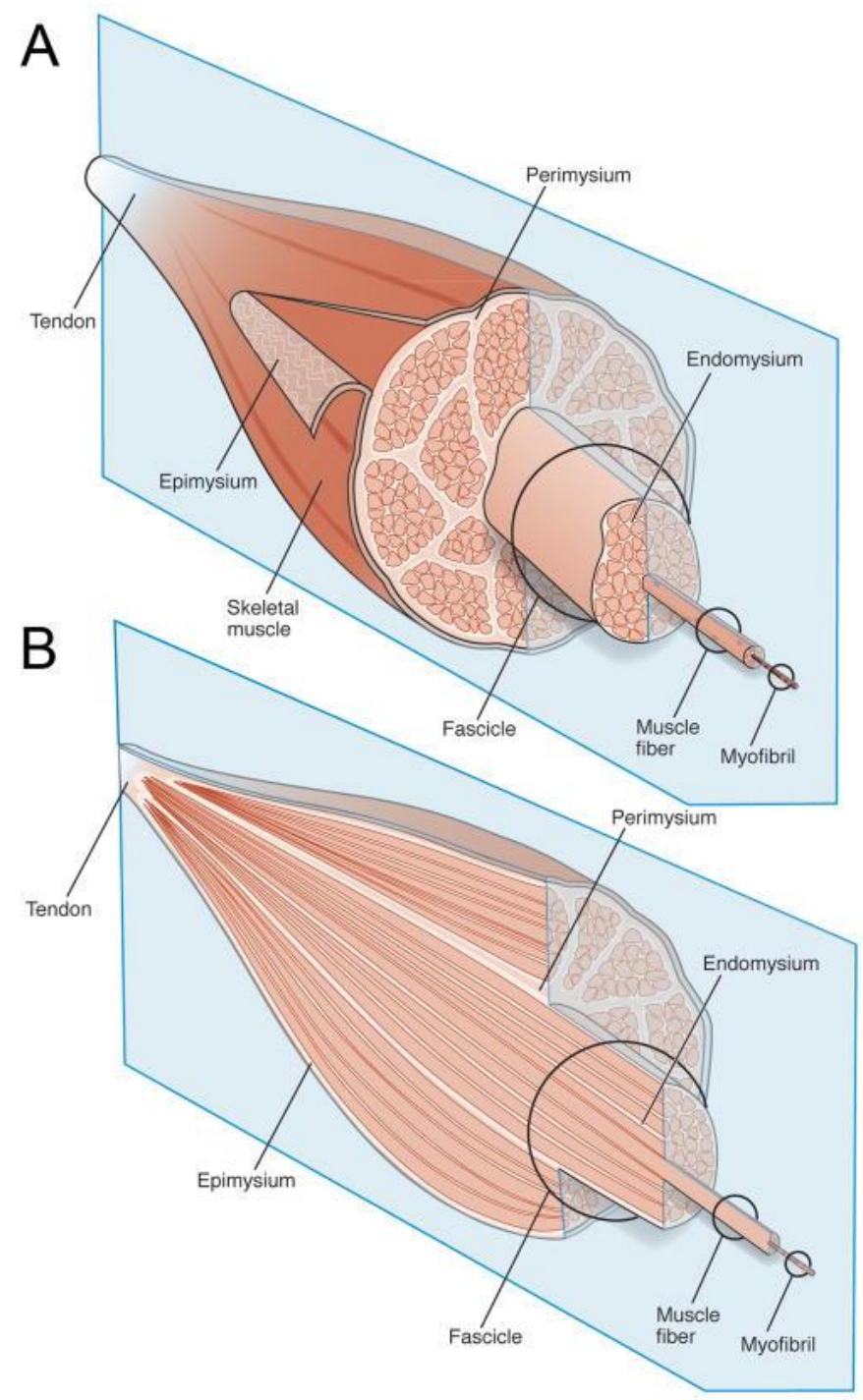

Figure I-3 Skeletal muscle and extracellular matrix organization. Originally published in Muscle Nerve (Gillies and Lieber, 2012)[92]. A) Schematic of skeletal muscle extracellular matrix organization. B) Cross section of skeletal muscle to show the continuous nature of the perimysium with the tendon. 


\section{Mechanical function}

As for its role in body mechanics, skeletal muscle contraction provides the power and force necessary for body movement, for maintenance of skeletal structure, body posture, and offers protection of internal organs [97]. Early work done by Huxley and Niedergerke and Huxley and Hanson in two, consecutive, 1954 Nature articles laid the foundation for what would become known as the sliding-filament model, observing that as contraction occurs, actin and myosin filaments do not change size despite the overall shortening of the sarcomere and deducing instead that the actin and myosin filaments were overlapping in an ATP-dependent manor[88,98]. This work laid the foundation leading to our current understanding of muscle contraction.

Muscle contraction is facilitated largely by the thin actin and thick myosin filaments overlapping one another in an ATP-dependent manner in response to a calcium signal[87,99-101]. The process by which muscle contractions occurs in response to this signal is referred to as excitation-contraction coupling[87,97,100]. Muscle contraction begins with the muscle fiber receiving a signal from a motor neuron at the neuromuscular junction which "excites" the muscle via propagation of an action potential terminating at the neuromuscular junction at which point acetylcholine is released into the synaptic cleft where it will bind to its receptor leading to a local depolarization of the muscle fiber and the opening of voltage gated sodium channels. This leads to further/ depolarization of the transverse tubules within the sarcolemma, resulting in the release of calcium from the sarcoplasmic reticulum[97,100]. Skeletal muscle can exist in an off (resting) or on (actively contracting) state. In the resting state, intracellular calcium concentration is less than $100 \mathrm{nM}$, and when calcium is released from the sarcoplasmic reticulum, the 
concentration increases to $\sim 20 \mathrm{uM}[100]$. This influx of calcium ultimately leads to contraction by exposing the myosin binding sites on the thin actin filaments. Actin contains the proteins troponin and tropomyosin, and in a resting muscle tropomyosin covers myosin binding sites. Calcium will bind the troponin causing a conformational change uncovering the myosin binding sites[87,100,102].

The uncovering of these binding sites leads to muscle contraction via a process referred to as cross-bridge cycling wherein the myosin heads actually appear to walk along the actin filaments via ATP hydrolysis[87,102]. This process exists in four main steps: 1) formation of the cross bridge is facilitated by the binding of myosin to its binding sites on actin, 2) the power stroke of the myosin head occurs releasing ADP and Pi, 3) a new molecule of ATP binds, and 4) ATP is hydrolyzed and the myosin head is cocked and ready to re-form the cross bridge with actin[97,100,102]. Muscle relaxation is facilitated by the dissociation of the myosin heads from actin filaments in addition to the reuptake of calcium by the sarcoplasmic reticulum, both relatively slow processes[100].

\section{Bone muscle crosstalk}

Osteogenesis imperfecta translates to imperfect bone formation and, as the name literally suggests, is a bone disease. However, as mentioned, $80 \%$ of patients with mild OI experience muscle force deficits, suggesting the wide prevalence of muscle weakness in addition to the bone fragility $[6,47,48]$. It is important to consider the impact that muscle weakness may have on further compromising the bone of OI patients. Until recently, the understanding of the relationship between these two organs has been limited to the context of physical forces and biomechanics[60,61,103]. In this instance, the muscle acts 
as a pully exerting force on the bone, acting as a lever to move the skeleton[103]. The understanding of this relationship has evolved over the past decade, with the current understanding incorporating the idea that both bones and muscles act as secretory organs communicating via biochemical signaling in addition to the historical understanding of mechanotransduction[60,61,103].

\section{Biomechanical}

The earliest understanding of the relationship between bone and muscle was that of their physical interactions, with early studies suggesting a correlation between muscle weight and bone mass[104]. This idea, initially beginning as Wolf's law, eventually leading to Frost's mechanostat theory, postulates that bone strength is tightly coupled to skeletal muscle action[57,105,106]. Significant evidence in the field of musculoskeletal health has corroborated this idea since its initial conceptualization in $1892[106,107]$. Countless studies evaluating the consequences of disuse or unloading on these two organs support the notion that muscle force and bone strength are tightly coupled[5,14,103,108-112]. A summary of selected studies highlighting the mechanical relationship of bone and muscle can be seen in Table I-2.

The ability of bone to remodel its geometry and size in response to the mechanical strain from muscle is primarily facilitated by the osteocyte, a mechanosensory cell[69]. For mechanotransduction to occur, the osteocyte must perceive a mechanical signal then convert it to a biochemical signal to elicit a response by osteoblasts and/or osteoclasts to facilitate bone remodeling[69,113-115]. The exact mechanisms by which the osteocytes act as a mechanosensing cells as well as the components of the osteocyte that facilitate 
mechanosensing, remain unknown, although there is evidence that the dendritic processes, cell body, and cilium contribute to the osteocytes' ability to function as a mechanosensory cell[ 69,114$]$. It is hypothesized that the osteocyte can respond to several factors in response to mechanical strain, including the physical deformation of the bone matrix, fluid flow shear stress, and streaming potentials $[69,114,116]$. 
Table I-2: Studies highlighting the mechanical relationship of muscle and bone.

\begin{tabular}{|c|c|c|c|}
\hline $\begin{array}{l}\text { Reference } \\
\text { (Author) }\end{array}$ & Title & $\begin{array}{l}\text { Area of } \\
\text { Research }\end{array}$ & Significant Findings \\
\hline $\begin{array}{l}\text { Scheld K. et } \\
\text { al., } 2001\end{array}$ & $\begin{array}{l}\text { Nitrogen } \\
\text { metabolism and } \\
\text { bone metabolism } \\
\text { markers in } \\
\text { healthy adults } \\
\text { during } 16 \text { weeks } \\
\text { of bed rest }\end{array}$ & Bed Rest & $\begin{array}{l}\text { Within } 2 \text { weeks of bed rest in healthy } \\
\text { adults, bone metabolic marker, } \\
\text { osteocalcin was decreased suggesting } \\
\text { reduced bone formation. Additionally, } \\
\text { body nitrogen was decreased within the } \\
\text { initial } 2 \text { weeks of bed rest which was } \\
\text { associated with a rapid decline in } \\
\text { muscle mass[117]. }\end{array}$ \\
\hline $\begin{array}{l}\text { Lang T. et } \\
\text { al., } 2004\end{array}$ & $\begin{array}{l}\text { Cortical and } \\
\text { trabecular bone } \\
\text { mineral loss from } \\
\text { the sine and hip in } \\
\text { long-duration } \\
\text { spaceflight }\end{array}$ & Microgravity & $\begin{array}{l}\text { Areal bone mineral density was } \\
\text { reduced in both spine and hip at rates } \\
\text { of approximately } 0.9 \% \text { and } 1.4 \% \text {, } \\
\text { respectively in } 14 \text { members aboard the } \\
\text { International Space Station that were } \\
\text { followed during their } 4 \text { to } 6 \text { month } \\
\text { flights[118]. }\end{array}$ \\
\hline $\begin{array}{l}\text { Novotny } \\
\text { SA. et al., } \\
2011\end{array}$ & $\begin{array}{l}\text { Bone is } \\
\text { functionally } \\
\text { impaired in } \\
\text { dystrophic mice } \\
\text { but less so than } \\
\text { skeletal muscle }\end{array}$ & $\begin{array}{l}\text { Musculoskeletal } \\
\text { Disease }\end{array}$ & $\begin{array}{l}\text { Mdx (dystrophin deficient) and dko } \\
\text { (utrophin deficient) mice exhibit } \\
\text { altered bone geometry and reduced } \\
\text { bone material properties including } 50 \% \\
\text { reduction in bone strength compared to } \\
\text { WT littermates[119]. }\end{array}$ \\
\hline $\begin{array}{l}\text { Tagliaferri } \\
\text { C. et al, } \\
2015\end{array}$ & $\begin{array}{l}\text { Muscle and bone, } \\
\text { two } \\
\text { interconnected } \\
\text { tissues }\end{array}$ & $\begin{array}{l}\text { Bed Rest, } \\
\text { Aging, } \\
\text { Microgravity }\end{array}$ & $\begin{array}{l}\text { A comprehensive review discussing the } \\
\text { consequences of reduced mechanical } \\
\text { loading providing a case that } \\
\text { mechanical loading links bone and } \\
\text { muscle functions[120]. }\end{array}$ \\
\hline $\begin{array}{l}\text { Yuan Y. et } \\
\text { al., } 2016\end{array}$ & $\begin{array}{l}\text { The roles of } \\
\text { exercise in bone } \\
\text { remodeling and in } \\
\text { prevention and } \\
\text { treatment of } \\
\text { osteoporosis }\end{array}$ & $\begin{array}{l}\text { Aging and } \\
\text { Exercise }\end{array}$ & $\begin{array}{l}\text { This thorough review summarizes the } \\
\text { effects that exercise can have on } \\
\text { different types of bone cells in terms of } \\
\text { their mechanical and signaling } \\
\text { responses to exercise as well as how } \\
\text { this translates prevention of age-related } \\
\text { effects of disuse such as } \\
\text { osteoporosis[121]. }\end{array}$ \\
\hline $\begin{array}{l}\text { Veilleux } \\
\text { LN. \& } \\
\text { Rauch F., } \\
2017\end{array}$ & $\begin{array}{l}\text { Muscle-Bone } \\
\text { Interactions in } \\
\text { Pediatric Bone } \\
\text { Diseases }\end{array}$ & $\begin{array}{l}\text { Musculoskeletal } \\
\text { Disease }\end{array}$ & $\begin{array}{l}\text { This review highlights the clinical } \\
\text { perspectives in regards to pediatric } \\
\text { patients on the roles of bone in muscle } \\
\text { disease and the role of muscle in bone } \\
\text { disease[5]. }\end{array}$ \\
\hline $\begin{array}{l}\text { Bettis T., } \\
\text { Kim B-J., } \\
\text { and Hamrick } \\
\text { MW., } 2018\end{array}$ & $\begin{array}{l}\text { Impact of muscle } \\
\text { atrophy on bone } \\
\text { metabolism and } \\
\text { bone strength: } \\
\text { implications for } \\
\text { muscle-bone } \\
\text { crosstalk with } \\
\text { aging and disuse }\end{array}$ & $\begin{array}{l}\text { Aging, Bed } \\
\text { Rest, and } \\
\text { Microgravity }\end{array}$ & $\begin{array}{l}\text { This review offers a comprehensive } \\
\text { overview of bone muscle crosstalk } \\
\text { including both biomechanical and } \\
\text { biochemical examples in clinical cases } \\
\text { and animal model studies focused on } \\
\text { the effects of inactivity or muscle } \\
\text { disuse specifically in instances of } \\
\text { aging, bed rest, and microgravity[111]. }\end{array}$ \\
\hline
\end{tabular}


In response to a mechanical stimulus, intracellular calcium levels increase and the osteocyte begins to regulate and integrate osteoblasts and osteoclasts to control bone remodeling[114]. To respond to mechanical stress, the osteocyte releases signals including nitrous oxide, prostaglandins, and ATP, which are believed to have direct effects on osteoblasts and osteoclasts, in response to this increased intracellular calcium[114]. One of the most important signaling pathways modulated by the osteocyte's response to mechanical stress is the Wnt/ $\beta$-catenin pathway[122,123].

A summary of osteocyte signaling on osteoblasts and osteoclasts in response to mechanical stimuli can be seen in Figure I- 4[114]. Briefly, prostaglandins, nitrous oxide, and ATP have a direct positive effect on bone formation, while nitrous oxide has a negative effect on osteoclasts, resulting in a net gain of bone[114]. Furthermore, members of the Wnt/ $\beta$-catenin signaling pathway, and thus bone formation, are inhibited via sclerostin, dickkopf-1 (DKK1), and selected frizzled-related protein 1 (sFRP1)[114,124]. Receptor activator of nuclear kappa-B ligand (RANKL), another important regulator in bone remodeling, activates the osteoclast resulting in increased bone resorption whereas osteoprotegerin (OPG) inhibits RANKL resulting in increased bone formation[114,124]. Finally, macrophage colony stimulating factor (M-CSF) regulates differentiation of osteoclasts from hematopoietic stem cells[124]. Importantly, several of these signaling molecules serve as targets of novel therapies being investigated in OI including the use of anti-sclerostin and RANKL inhibitor antibodies[15]. 


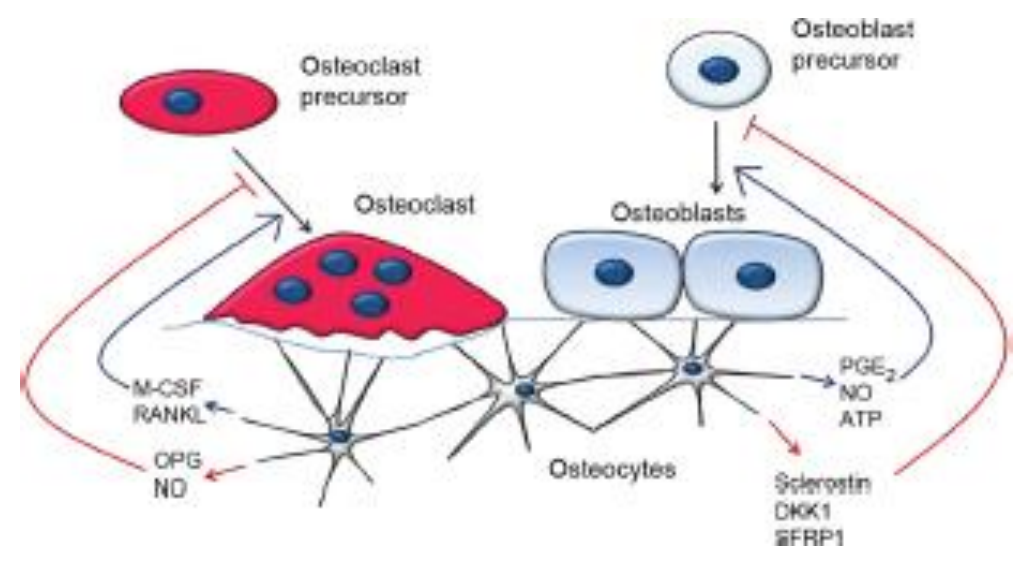

Figure I-4 Osteocyte regulation of bone remodeling. Originally published in Endocrine Reviews (Dallas et al., 2013)[114]. The osteocytes secrete factors that act on osteoblast and osteoclast precursors to modulate bone remodeling.

Fifty percent of peak adult bone mass is accrued during adolescence and there is strong evidence that $20-40 \%$ of adult peak bone mass is determined by modifiable factors including exercise, further reiterating the importance of exercise in children with OI[125,126]. Studies have demonstrated that children with OI have activity levels comparable to those of their healthy peers, and there is evidence that increased physical activity may also improve aerobic capacity and muscle strength $[47,115,127,128]$.The use of exercise and whole body vibration therapy in OI as modalities of treatment to exploit the biomechanical relationship between bone and muscle have also been investigated[14].

Whole body vibration therapy (WBV) has also been implemented as treatment in OI and is an especially attractive target for children with severe OI and lower ambulation and ability to participate in traditional physical activites [129-131]. Studies in the oim mouse evaluating the use of WBV demonstrated improvement of several bone parameters including increased cortical bone area, cortical thickness, and trabecular bone volume while no significant changes in bone mechanical properties were observed [130]. Limited 
studies on the use of WBV in OI exist and the results from these studies are conflicting with one study by Hoyer-Kuhn et al. demonstrating improved bone mineral density and motor function in patients with OI while another study by Högler et al. demonstrated increased lean mass, but no changes in bone mass or muscle function[129,132]. This discrepancy and inability of OI patients to respond to WBV may be the result of genetic mutations specificity or differing levels of OI severity or may reflect a reduced biomechanical responsiveness of OI bone[132]. Regardless, the use of exercise and WBV as therapeutic approaches in OI are attractive targets that are minimally invasive and cost-effective ways to exploit the biomechanical relationship of bone and muscle.

\section{Biochemical}

Until recently, the nature of bone-muscle crosstalk was thought to be limited to the biomechanical relationship. However, more recent research has highlighted the presence of extensive biochemical crosstalk that occurs with both bone and muscle acting as secretory and endocrine organs[60,61,133]. One of the earliest reports suggesting biochemical crosstalk between muscle and bone, specifically a positive effect of muscle tissue on osteogenesis independent of contraction, demonstrated improved fracture healing (increased speed and quality) when an open tibial fracture was wrapped with a muscle flap relative to non-wrapped tibias in a canine model[134]. This has been reproduced in rodent models by showing improved tibia open fracture healing in the presence of a muscle flap compared to a fasciocutaneous flaps[135]. Since these findings, the field of musculoskeletal interaction research has grown exponentially, with roughly 600 articles published in 2019 [136]. 
Proposed musculoskeletal biochemical interactions can be summarized into three main mechanisms[136]. The first two mechanisms suggest that both bone and muscle secrete soluble factors into the blood stream due to their highly vascularized nature[136]. The third mechanism consists of direct cell-to-cell contact; due to the close proximity of bone and muscle, it is likely that small molecules are able to be directly signaled between the two organs via extracellular vesicles or passive diffusion [136]. These factors secreted by muscle and bone are referred to as myokines and osteokines, respectively.

Myostatin, discovered in 1997, was the first and remains the best characterized myokine, although the term myokine was not coined until 2003, by Pedersen et al[133,136-138]. Since then, over 3,000 myokines have been identified[139]. Additionally, there is evidence that different myokines may be preferentially secreted by certain types of muscle fibers (i.e.glycolytic vs. oxidative)[139,140]. Some of the most prominent myokines, especially in the context of muscle-bone crosstalk include myostatin (mstn), interleukin-6 (IL-6), irisin, and $\beta$-aminoisobutyric acid (BAIBA) $[14,61,136,140]$. Just as muscle acts as a secretory organ releasing myokines that will act on bone, bone reciprocates this action, releasing osteokines that act on muscle including osteoclacin, Wnt3a, TGF $\beta$, and sclerostin[14,136]. The role of these myokines and osteokines are summarized in Tables I-3 and 1-4, respectively. Several examples of altered biochemical signaling pathways in OI muscle and bone have been described and novel therapeutics have emerged acting on these altered pathways. 
Table I-3: Myokines and their roles on bone

\begin{tabular}{|l|l|}
\hline Myokine & Role in bone \\
\hline $\begin{array}{l}\text { Myostatin } \\
\text { (mstn) }\end{array}$ & $\begin{array}{l}\text { A member of the TGF- } \beta \text { superfamily and negative regulator of } \\
\text { muscle mass. In addition to its inhibitory effect on muscle growth, } \\
\text { mstn has been repeatedly shown to have a negative impact on bone } \\
\text { formation via increased osteoclastogenesis, increased osteocyte } \\
\text { expression of negative regulators of bone including sclerostin, and } \\
\text { reduced expression of osteoblast differentiation } \\
\text { markers[133,136,138,141-143]. }\end{array}$ \\
\hline $\begin{array}{l}\text { Interleukin-6 } \\
\text { (IL-6) }\end{array}$ & $\begin{array}{l}\text { Although secreted by multiple tissues, large amounts of IL-6 are } \\
\text { secreted by muscle in response to exercise leading to its } \\
\text { characterization as a myokine[137]. Interestingly, IL-6 has been } \\
\text { shown to upregulate both osteoclast and osteoblast } \\
\text { formation[136,144,145]. }\end{array}$ \\
\hline Irisin & $\begin{array}{l}\text { Irisin has been shown to be positively correlated with BMD and } \\
\text { negatively correlated with serum sclerostin[146,147]. Additionally, } \\
\text { in mice, treatment with recombinant irisin led to improved bone } \\
\text { geometry and mechanical properties[148]. }\end{array}$ \\
\hline $\begin{array}{l}\text { B- } \\
\text { aminoisobutyric } \\
\text { acid (BAIBA) }\end{array}$ & $\begin{array}{l}\text { BAIBA, a myokine secreted in response to exercise was originally } \\
\text { identified for its function in the browning of white adipose tissue } \\
\text { though more recent studies have demonstrated a role in osteocytes as } \\
\text { a protective agent against reactive oxygen species[149,150]. }\end{array}$ \\
\hline
\end{tabular}

Table I-4: Osteokines and their roles on muscle

\begin{tabular}{|l|l|}
\hline Osteokine & Role in muscle \\
\hline $\begin{array}{l}\text { Osteocalcin } \\
(\text { OCN })\end{array}$ & $\begin{array}{l}\text { OCN, secreted by the osteoblast plays an important role in glucose and } \\
\text { energy homeostasis and is believed to promote nutrient catabolism and } \\
\text { uptake in skeletal muscle as well as improve exercise capacity[151- } \\
153] .\end{array}$ \\
\hline Wnt3a & $\begin{array}{l}\text { Wnt3a, secreted by osteocytes, was shown to promote myogenesis via } \\
\text { increased myogenin and myoD expression in a cultured osteocyte cell } \\
\text { line }(\mathrm{C} 2 \mathrm{C} 12)[154,155] .\end{array}$ \\
\hline $\begin{array}{l}\text { Transforming } \\
\text { growth } \\
\text { factor- } \beta \\
\text { (TGF- } \beta)\end{array}$ & $\begin{array}{l}\text { TGF- } \beta \text {, an osteokine secreted by osteoblasts has been shown to } \\
\text { negatively impact skeletal muscle function via calcium leakage and } \\
\text { increased oxidative stress[156,157]. }\end{array}$ \\
\hline Sclerostin & $\begin{array}{l}\text { Although there is not evidence of sclerostin, secreted by the osteocyte, } \\
\text { directly effecting muscle; it has been shown to inhibit Wnt-3a action in } \\
\text { skeletal muscle, thus indirectly negatively impacting } \\
\text { muscle[154,158,159]. }\end{array}$ \\
\hline
\end{tabular}


The role of these osteokines in osteogenesis imperfecta have been well characterized, while less information exists regarding the role of different myokines in the pathology of OI. Of the myokines, myostatin has been the most studied in the context of OI. Myostatin is a negative regulator of muscle mass that signals through the activin receptor type IIB to inhibit muscle growth[160]. Several studies in the oim and $+/ G 610 C$ mouse models have highlighted the potential for myostatin inhibition to have a beneficial outcome on bone properties. The first report highlighting the benefit of myostatin (mstn) inhibition demonstrated that +/oim mice, modeling a mild to moderate patient OI, when also deficient in mstn $(+/$ oim $+/$ mstn $)$, exhibited improved femoral bone biomechanical properties compared to their +/oim littermates[161]. Other data suggest that the responsiveness to myostatin inhibition is OI mutation-specific [162,163]. Studies using the activin receptor IIB decoy molecule resulted in improved skeletal properties in the +/G610C mouse, modeling a mild to moderate human OI, and improved skeletal muscle contractile properties in the oim/oim mouse[162,163]. These studies suggest that myostatin may serve as a novel therapeutic target in the treatment of OI, although responses may be based on specific mutations.

As indicated above, relative to the myokines, more data exists on osteokines in OI. Osteocalcin levels are elevated in both patients and the Collal $^{+/ J r t}$ OI mouse model $[8,164,165]$. Additionally, dysregulated TGF- $\beta$ signaling has been observed in OI mouse models with increased signaling observed in both recessive $\left(\mathrm{Crtatp}^{-/}\right)$and autosomal dominant $\left(+/ G 610 C\right.$ and Collal $\left.{ }^{+/ J t}\right)$ variants of the disease[15,22,166]. However, treatment with a TGF- $\beta$ neutralizing antibody only improved bone properties in two of these models (Crtap-/- and Collal $\left.{ }^{+/ J r}\right)$ suggesting the importance of mutation specific 
treatment approaches $[15,22,166]$. Clinical trials in OI patients are also ongoing using TGF- $\beta$ antibody[2]. Finally, there is not a clear consensus on whether or not the levels of sclerostin are altered in OI patients, although there is evidence of improved bone properties in several mouse models of OI (oim/oim[24], +/G610C, Brtl-/-[167], Colla1 $^{+/ J r t}[168]$, and Crtap-/-[169]) and patient clinical trials utilizing sclerostin antibody are ongoing, with initial results showing increased markers of bone formation and reduced markers of bone resorption $[2,14,15,170,171]$.

The relationship between bone and muscle is highly synergistic, exhibiting both biomechanical and biochemical crosstalk. Biomechanical and biochemical crosstalk are not mutually exclusive. The role of myostatin in bone muscle crosstalk highlights this point. Inhibition of the myokine, myostatin, leads to altered mechanotransduction via increased muscle size; in addition this inhibition has a direct effect on biochemical signaling leading to a downregulation of osteoclasts. Understanding the complexity of bone muscle crosstalk in OI is crucial to determining novel therapies that can exploit this relationship by improving muscle function to ultimately improve bone strength.

\section{$\underline{\text { Skeletal muscle pathology in disease }}$}

Skeletal muscle weakness arises as part of the pathology of cancer cachexia, numerous congenital myopathies and neuromuscular diseases, and with aging[172-175]. Much research has been compiled on each of these individual instances, but there is not a single mechanism that leads to muscle weakness. Furthermore, the need for research in OI skeletal muscle weakness is apparent due to the lack of studies aiming to understand skeletal muscle weakness mechanisms in OI. Thus, it is necessary to review the 
mechanisms of muscle weakness in other diseases to get an understanding of how muscle weakness might arise in OI.

One field with extensive research is that of the muscular dystrophies including Duchenne and Becker Muscular dystrophies, both arising from mutations in the dystrophin gene, as well as other congenital muscular dystrophies which include Ullrich syndrome and Bethlem myopathy, arising from mutations in the type VI collagen genes[175]. In Duchenne and Becker muscular dystrophies, the absent or highly reduced amount of dystrophin disrupts skeletal muscle organization, sarcolemma fragility, and abnormal calcium homeostasis [176-178]. Excessive calcium and damaged mitochondria have been observed in the dystrophic muscle with damaged mitochondria further perpetuating calcium dysregulation, ultimately resulting in increased damage of mitochondria in a feed-forward fashion[176]. Similarly, the mutations in type VI collagen genes can lead to disruptions in the extracellular matrix (specifically the perimysium and endomysium [179]) and increased apoptosis via mitochondrial dysfunction[180,181]. Mitochondrial dysfunction has also been implicated in aging and neurodegenerative disorders[182,183], so while there is no single mechanism that leads to skeletal muscle weakness, mitochondrial dysfunction appears a common theme amongst these different instances of skeletal muscle weakness.

\section{Mitochondria: the powerhouse of the cell?}

Mitochondria are commonly and simply referred to as the powerhouse of the cell, and although they were originally known for their role in oxidative phosphorylation and ATP production, mitochondria are also crucial in other cellular functions including calcium 
homeostasis, cell signaling, ER stress, autophagy, apoptosis, and others[184].

Mitochondria are double membrane- bound organelles that possess their own circular, double stranded DNA encoding members of the electron transport chain necessary for oxidative phosphorylation[185,186]. Several parameters dictate mitochondrial function and health including mitochondrial dynamics and morphology as well as mitochondrial biogenesis and mitophagy[184,186,187].

A textbook image of a mitochondrion often highlights its spherical shape with a welldefined outer and inner membrane, as well as the presence of membrane invaginations known as cristae. However, a mitochondrion's morphology is more complex and is regulated by the dynamic nature facilitated by mitochondrial fusion and fission in response to a cell's unique needs[188]. Because mitochondrial morphology and function are tightly coordinated, mitochondria can exhibit unique morphology from cell to cell with mitochondria in skeletal muscle consisting of a network of interconnected mitochondria, although recent studies suggest mitochondrial morphology in skeletal muscle may be more complex than this, with the network and interconnectedness varying across the muscle[184,186,189]. Mitochondrial fusion is the union of two mitochondria, often allowing for the combination of two smaller organelles to ultimately increase the size of the mitochondrial network, and is facilitated by the proteins mitofusin 1 and 2, as well as optical atrophy 1 and 2[184,186]. Mitochondrial fission is the fragmentation of mitochondria often in response to damage and is facilitated by the proteins dynaminrelated protein 1 , mitochondrial fission factor, and fission protein $1[184,186]$.

Mitochondrial biogenesis and mitophagy are coordinated with the mitochondrial dynamics and also play critical roles in overall mitochondrial health by controlling 
production of new mitochondria and mitochondrial clearance, respectively[186,187]. The process of mitochondrial biogenesis and mitophagy involve complex regulation via different signaling pathways. Increased intracellular calcium is one of the most common activators of mitochondrial biogenesis, leading to activation of AMP-activated protein kinase and ultimately PGC1- $\alpha$ whom is considered the "master regulator" of mitochondrial biogenesis[187]. Briefly, activation of PGC1- $\alpha$ along with its co-activators NRF-1 and NRF-2 lead to activation of TFAM, a mitochondrial transcription factor leading to mitochondrial DNA transcription and replication[190]. Mitophagy is a form of selective autophagy often initiated in response to damaged or dysfunctional mitochondria[187,191]. Two major pathways of mitophagy are the PINK-1/Parkin ubiquitin pathway and the BNIP3/NIX pathway[187,191]. In the PINK-1/Parkin dependent pathway, PINK-1 is recruited to a depolarized mitochondrial membrane at which point Parkin, an E3 ubiquitin ligase, is recruited ultimately leading to the mitophagy[191]. BNIP3 and NIX are both atypical members of the BCL2 family, a group of proteins regulating cell death[191]. These proteins through there interaction with LC3, an important component of autophagosome formation, lead to degradation of the mitochondria[191].

As previously discussed, the roles of mitochondria in cell health and maintenance are diverse. Briefly, their well-known roles in ATP production and calcium homeostasis will be discussed. The mitochondrial membrane is the site where the respiratory chain carries out oxidative phosphorylation, the process of electron transport ultimately leading to ATP production[192]. The process of oxidative phosphorylation includes the transfer of electrons through complexes I-IV of the electron transport chain with $\mathrm{O}_{2}$ as the terminal 
electron acceptor. During this process protons are pumped across the membrane generating an electro-chemical gradient which is used to drive ATP synthesis via complex V (ATP synthetase) (Figure I-5) [192]. Calcium signaling is an important regulator of many different cellular processes and the mitochondria aid in regulating calcium homeostasis by serving as a reservoir for taking up extracellular calcium, although excessive calcium influx can lead to mitochondrial damage and dysfunction[193].

In skeletal muscle, three distinct populations of mitochondria exist: the intermyofibrillar mitochondria, subsarcolemmal mitochondria, and a third less characterized yet distinct population extending from the subsarcolemmal mitochondria[194]. These distinct populations differ in terms of location, morphology, and function and have been shown to be differentially affected in disease states[186,194]. The subsarcolemmal mitochondria lie beneath the mitochondrial membrane and are believed to play roles in ATP generation necessary for membrane transport whereas the intermyofibrillar mitochondria are located near Z-lines in skeletal muscle and are believed to generate ATP necessary for contraction and regulate calcium signaling[186]. Due to their diverse cellular roles, regulation of their distinct mitochondrial content and function is essential for skeletal muscle health. 


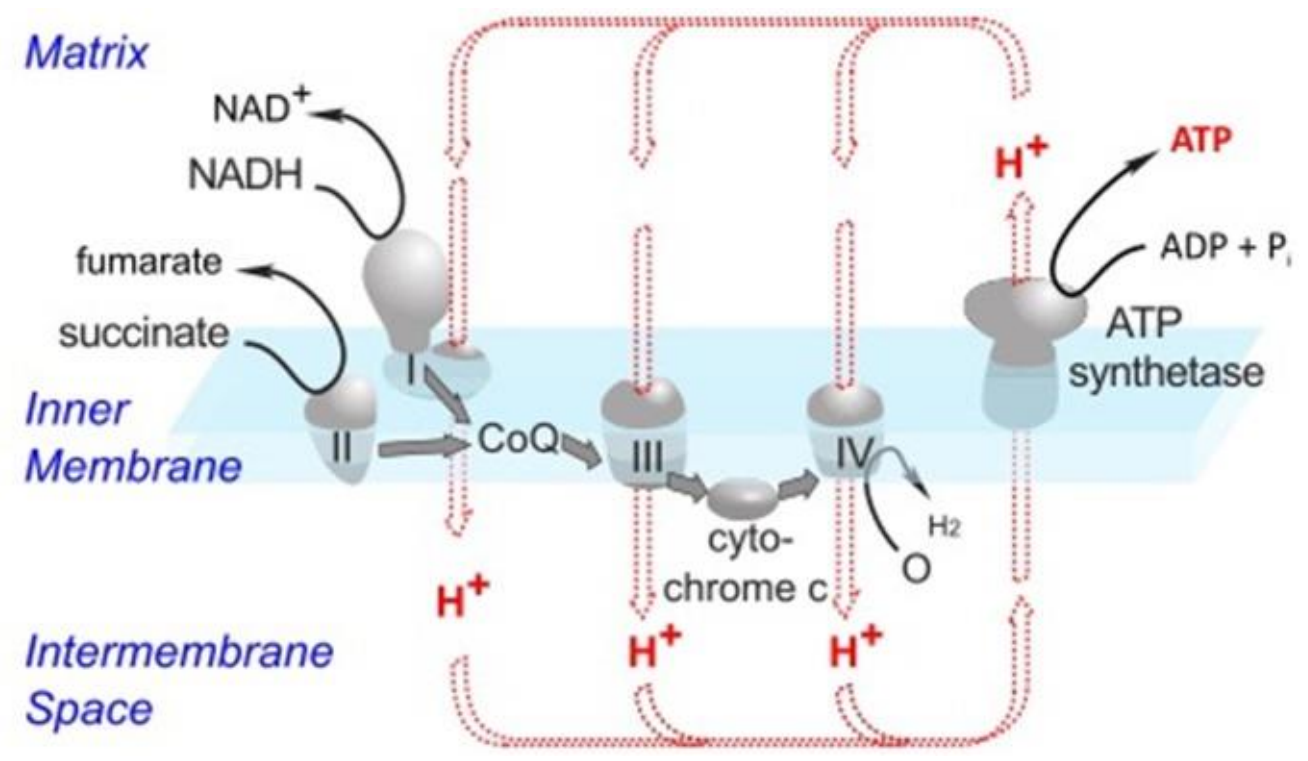

Figure I-5 Oxidative Phosphorylation. Originally published in Genetics (van der Bliek et al., 2017)[192]. Electrons are shuttled through electron transport chain complexes I-IV to oxygen, the final electron acceptor. This process generates a proton gradient that drives ATP synthesis via protonmotive force. 


\section{Links between collagen and mitochondrial function}

While there is no clear link between type I collagen and mitochondrial function, a link between type VI collagen and muscle mitochondrial function can be seen in cases of Bethlem myopathy and Ullrich Congenital Muscular dystrophy[180,181,195]. In these cases, type VI collagen defects result in a mitochondrial myopathy believed to be the result of induction of mitochondrial permeability transition (the rapid increase of inner membrane permeability to solutes[196]) due to the opening of the mitochondrial permeability transition pore (mPTP), a high conductance channel whose components and structure remain to be elucidated[180,197]. Under normal circumstances, the mPTP functions in the "direction" of ATP synthesis with occasional transient opening facilitating regulated apoptosis[198]. However, prolonged opening, the result of excessive calcium stress, reactive oxygen species or a combination of the two, can cause dissipation of the proton gradient and uncoupling of oxidative phosphorylation leading to mitochondrial dysfunction[198]. In animal studies and clinical trials, cyclosporine A, an inhibitor cyclophilin $\mathrm{D}$ (an activator of the $\mathrm{mPTP}$ ) has shown promising results improving the mitochondrial dysfunction associated with type VI collagen defects by inhibiting mitochondrial permeability transition [181,199-202].

While there is no clear link between type I collagen and mitochondrial function, there is limited evidence of interactions between type I and type VI collagen, which may imply an indirect relationship between type I collagen and mitochondrial function. An early report demonstrates the binding of type I collagen to the $\alpha 3$ chain of type VI collagen in chicken connective tissue[203]. Furthermore, an in silico analysis of the mouse $\alpha 3$ chain of type VI collagen (Col6a3) on the STRING: functional protein association network 
database reveals that the type I collagen genes, Collal and Colla2, are amongst the top five predicted interaction partners (https://string-db.org). Type VI collagen is an important extracellular matrix protein found in many diverse tissues including skeletal muscle[195]. In skeletal muscle, the extracellular matrix plays important role in maintaining structure and lateral force transmission throughout the muscle[204]. The collagen network of the perimysium is composed of mainly type I collagen with types III, VI, and XII present in lesser amounts[205]. Defects in type I collagen may lead to destabilization of the perimysial collagen bundles and ultimately the perimysial junction plate, a region important for lateral force transmission where the permysial collagen bundles interact with the endomysium[205]. Moreover a recent study of teriparatide (a bone anabolic) treatment in OI patients and post-menopausal women revealed increased presence of collagen biomarkers including types II, III, IV, V, and VI in OI patients, but not in post-menopausal women[206]. This further suggests the possibility of dysregulated extracellular matrix interactions as part of the muscle pathology in OI.

\section{$\underline{\text { Research Goals and Objectives }}$}

Skeletal muscle weakness is a prominent manifestation in OI with over $80 \%$ of mild patients exhibiting muscle force deficits and the prevalence of muscle weakness correlating with severity of disease. The wide occurrence of skeletal muscle weakness in OI, limited studies aimed at understanding this weakness, and lack of therapeutic options targeting skeletal muscle weakness in OI demonstrate the need for further research evaluating muscle function and mechanisms of skeletal muscle weakness in OI. The goal of this work was to begin to develop an understanding of the skeletal muscle weakness observed in OI using the oim/oim mouse, modeling severe human type III OI, to evaluate 
mechanisms underlying skeletal muscle weakness. We focused our studies on the role of mitochondrial health, hypothesizing that abnormal mitochondria in the oim/oim skeletal muscle would adversely impact skeletal muscle function.

The first aim of my study, described in chapter II, was to characterize mitochondrial content and function in oim/oim mouse skeletal muscle. Prior to this work, citrate synthase activity was determined to be increased in oim/oim whole gastrocnemius muscle relative to WT littermates suggesting an increase in mitochondrial content. Here, we demonstrate that mitochondrial function is significantly reduced in isolated oim/oim gastrocnemius mitochondria evidenced by significantly reduced mitochondrial respiration rates and significantly reduced mitochondrial citrate synthase activity. Furthermore, we found glycogen content to be equivalent between oim/oim and WT gastrocnemius muscles, further indicating that reduced mitochondrial function rather than another mechanism, such as changes in substrate preferences, was likely contributing to skeletal muscle weakness. Additionally, we observed reduced mitochondrial DNA to nuclear DNA ratios, increased protein content of markers of mitochondrial biogenesis (PGC1- $\alpha$ and TFAM), and reduced protein content of LC3, an important marker of mitophagy in the skeletal muscle of oim/oim mice relative to WT littermates. This study was the first to characterize mitochondrial function in a mouse model of OI and will hopefully stimulate new research in the field regarding the role of mitochondria in OI skeletal muscle weakness.

In chapter III, I describe the second aim, the further characterization of mitochondrial function in liver and cardiac muscle in addition to the evaluation of several parameters of whole body and skeletal muscle metabolism in the oim/oim mouse. One of the most 
significant findings of this study was that mitochondrial dysfunction in the oim/oim mouse is not global, as the mitochondrial dysfunction was present in oim/oim gastrocnemius mitochondria, but not in liver or heart mitochondria. We also observed reduced complete and incomplete fatty acid oxidation in oim/oim gastrocnemius mitochondria, but not in oim/oim whole quadriceps muscle, further confirming the presence of a mitochondrial dysfunction and suggesting a compensatory effect in the whole muscle. Moreover, we noted increased whole-body energy expenditures consistent with previous reports in the Collal ${ }^{J R T /+}$ mouse and OI patients. We also saw reduced production of $\mathrm{H}_{2} \mathrm{O}_{2}$ in oim/oim gastrocnemius mitochondria and a change in soleus muscle fiber type distributions with a decrease in the percentage of slow twitch type I fibers and an increase in the percentage of fast twitch type IIa fibers in oim/oim mice relative to WT. Completion of this study further characterized skeletal muscle specific mitochondrial dysfunction in the oim/om mouse and demonstrated the potential of a metabolic phenotype in the oim/oim mouse.

Finally, chapter IV provides a brief summary of the work presented in this dissertation followed by three primary objectives that are necessary to move the field forward.

The aims of this research were to 1) characterize mitochondrial function in the hindlimb muscles of a mouse modeling a severe human type III OI (oim/oim), 2) determine if mitochondrial dysfunction is a global phenomenon in the oim/oim mouse, and 3) determine if whole body and skeletal muscle metabolic parameters are altered in the oim/oim mouse. To this end, we discovered that 1) oim/oim mice exhibit severe skeletal muscle mitochondrial dysfunction evidenced by drastic reductions in mitochondrial respiration and mitochondrial citrate synthase activity, 2) this mitochondrial dysfunction 
is not global as reduced mitochondrial respiration is not observed in heart or liver tissue, and finally, 3) a subtle metabolic phenotype is present in the oim/oim mice including the presence of altered body composition and increased energy expenditure.

This work contributes to the understanding of muscle weakness in OI by beginning to investigate the role of mitochondrial function in the oim/oim mouse. These results should stimulate further research into mechanisms of muscle weakness in patients and mouse models of OI highlighting the important need for future research into mitochondrial health and metabolism in OI. Elucidation of these mechanisms could potentiate novel therapeutic targets aimed at improving skeletal muscle function which, due to the highly synergistic nature of muscle and bone, would ultimately lead to improved bone strength. 
CHAPTER II

COMPROMISED EXERCISE CAPACITY AND MITOCHONDRIAL

DYSFUNCTION IN THE OSTEOGENESIS IMPERFECTA MURINE (OIM) MOUSE

MODEL

This chapter has been published in the Journal of Bone and Mineral Research

Gremminger VL, Jeong Y, Cunningham RP, Meers GM, Rector RS, Phillips CL. Compromised Exercise Capacity and Mitochondrial Dysfunction in the Osteogenesis Imperfecta Murine (oim) Mouse Model. J Bone Miner Res. 2019 Sep;34(9):1646-1659. doi: 10.1002/jbmr.3732. Epub 2019 Jun 13. PMID: 30908713; PMCID: PMC6744299.

Victoria L Gremminger ${ }^{1}$, Youngjae Jeong ${ }^{1}$, Rory P. Cunningham ${ }^{3}$, Grace M. Meers ${ }^{3}$, R. Scott Rector ${ }^{3}$, Charlotte L. Phillips ${ }^{1,2^{*}}$

${ }^{1}$ Department of Biochemistry, University of Missouri, Columbia, Missouri, 65211

${ }^{2}$ Department of Child Health, University of Missouri, Columbia, Missouri, 65211

${ }^{3}$ Departments of Nutrition and Exercise Physiology and Medicine-GI, University of Missouri; Research Service-Harry S Truman Memorial VA Hospital, Columbia, Missouri, 65201 


\begin{abstract}
Osteogenesis imperfecta (OI) is a heritable connective tissue disorder that most often arises from type I collagen, COL1A1 and COL1A2, gene defects leading to skeletal fragility, short stature, blue-gray sclera, and muscle weakness. Relative to the skeletal fragility, muscle weakness is much less understood. Recent investigations into OI muscle weakness in both patients and mouse models have revealed the presence of an inherent muscle pathology. Understanding the mechanisms responsible for OI muscle weakness is critical, particularly in light of the extensive cross-talk between muscle and bone via mechanostransduction and biochemical signaling. In the following study we initially subjected wild-type (WT) and oim/oim mice, modeling severe human OI type III, to either weight -bearing (voluntary wheel running) or non-weight bearing (swimming) exercise regimens as a modality to improve muscle strength and ultimately bone strength. The oim/oim mice ran only $35-42 \%$ of the distance ran by age and sex-matched WT mice and exhibited little improvement with either exercise regimen. Upon further investigation, we determined that oim/oim gastrocnemius muscle exhibited severe mitochondrial dysfunction as characterized by $52-65 \%$ decrease in mitochondrial respiration rates, alterations in markers of mitochondrial biogenesis, mitophagy, and the electron transport chain components, as well as decreased mitochondrial citrate synthase activity, relative to age and sex-matched WT gastrocnemius muscle. Thus, mitochondrial dysfunction in the oim/oim mouse likely contributes to the compromised muscle function and reduced physical activity levels.
\end{abstract}




\section{Introduction}

Osteogenesis imperfecta (OI) is a heritable connective tissue disorder characterized most often by bone fragility, short stature, blue-gray sclera, and muscle weakness[1,17]. OI is a heterogeneous disease, both in phenotype and genotype that ranges in severity and can be classified into four main types: I-IV, and at least nine subtypes[1,13]. Type II is perinatal lethal; type III is the most severe viable form with patients often non-ambulatory, while patients with Types I and IV experience more mild and moderate manifestations, respectively. Roughly $85 \%$ of OI cases are the result of autosomal dominant mutations in the genes that encode the alpha chains of type I collagen (COL1A1 and COL1A2), while the remaining cases are due to rare mutations of genes primarily involved in type I collagen post-translational modifications and folding, bone mineralization, and osteoblast differentiation[1,17].

In addition to the skeletal fragility, patients with OI often experience muscle weakness and exhibit reduced cardiopulmonary fitness[46,48,207]. Both muscle weakness and reduced cardiopulmonary fitness in OI are less characterized compared to other manifestations, and whether they reflect an inherent pathology or are the result of inactivity is only beginning to be elucidated[115,208]. Recent investigations have begun to unveil the nature of the OI muscle weakness in both patients and mouse models, including recognition that approximately $80 \%$ of patients with type I OI due to mutations in the type I collagen genes exhibit muscle force deficits[4,6].

In humans, peak bone mass, the amount of bone accrued at the end of skeletal maturity, is achieved at or around the age of 30 with dramatic accumulation of bone during the pre- 
pubertal/pubertal growth period and then gradual decline initiating around 50 years of age[209]. Bone mass and size are largely attributed to heritable factors, as well as environmental factors, such as activity levels and hormonal and nutritional status. Currently, there is no cure for OI, and treatment options for OI patients are limited primarily to anti-resorptive bisphosphonate therapy and surgical rodding $[17,210]$. Although the contribution of environmental factors to bone strength are not as great as genetic factors, they are postnatally modifiable. Specifically, physical exercise has been suggested as one of the safest therapeutic options to enhance bone strength due to the mechanosensitive characteristics of bone. Several studies have demonstrated increases in bone mineral density (BMD) and bone mineral content (BMC) in more physically active children or children who underwent an exercise regimen[211,212]. Increased BMD has been attributed partly to increases in muscle mass and strength where muscle and bone mass are positively associated. Van Brussel et al. demonstrated improved aerobic capacity and muscle force in OI patients following a physical training regimen which suggests that exercise may be a viable treatment option for patients with mild and moderate OI[115].

In young and mature rodents, treadmill/voluntary wheel running exercise improved BMD, bone microarchitecture and biomechanical strength[213-218], and was protective against estrogen deficiency induced bone loss[216,217]. Non-weight bearing swimming exercise also prevented bone loss associated with ovariectomy or hindlimb suspension[219-221]. In the following study we investigated the effect of weight bearing (voluntary wheel running) and non-weight bearing (swimming) physical activity on musculoskeletal mass and strength in wildtype (WT) and homozygous osteogenesis 
imperfecta murine (oim/oim) mice. Gentry et al. was the first to identify an intrinsic muscle pathology in OI by demonstrating that oim/oim mice have reduced specific muscle contractile function (described as the peak tetanic force normalized to myofiber cross sectional area) as compared to WT littermates[3]. The oim/oim mice model moderately severe human OI type III due to a nucleotide deletion in the Colla2 gene leading to a frameshift resulting in non-functional $\alpha 2$ (I) chains and the formation of homotrimeric type I collagen, $\alpha 1(\mathrm{I})_{3}$, rather than the normal heterotrimeric type I collagen, $\left[\alpha 1(\mathrm{I})_{2} \alpha 2(\mathrm{I})\right][39]$. We hypothesized that wildtype and oim/oim mice that underwent 1) voluntary wheel running would exhibit increased skeletal mass and strength (ultimate force) as previously seen in C57BL mice[214], and 2) a swimming regimen would exhibit increased skeletal stiffness and sheer modulus of elasticity, with less of an impact on ultimate force[222,223]. We demonstrate that the present exercise regimens were unable to improve either muscle or bone strength in the oim/oim mice compared to their in-cage control oim/oim mice, even though WT mice demonstrated a positive correlation between distances ran and bone strength.

In addition to the severe bone phenotype, oim/oim mice exhibit decreased activity levels, muscle weights, reduced contractile force and elevated skeletal muscle citrate synthase activity compared to age and sex matched WT littermates[3,163]. The presence of altered citrate synthase activity and the recent report of altered energy metabolism in another moderately-severe OI mouse model, Col1a $1^{J t / /}$, which in younger mice exhibited reduced physical activity levels with increased oxygen consumption and $\mathrm{CO}_{2}$ production concomitant with increased whole body energy expenditure, and altered energy homeostasis[8], led us to investigate skeletal muscle mitochondrial bioenergetics in the 
oim/oim mouse. In the second half of this study, we demonstrate that oim/oim mouse skeletal muscle has poor mitochondrial respiration, decreased mitochondrial citrate synthase activity, and decreased mitochondrial DNA content in addition to alterations in mitochondrial electron transport chain (ETC) complex IV, markers of mitochondrial biogenesis, and markers of autophagy and mitophagy, suggesting a potential and critical role of mitochondria in the muscle weakness associated with OI.

\section{Methods}

\section{Animals}

Col1a $2^{\text {oim }}$ mice (stock \#001815) are publicly available from Jackson Laboratory (Bar Harbor, ME, USA). All mice were maintained on the C57BL/6J background and genotyped as previously described[39,224]. Mice were housed in an AAALACaccredited facility at the University of Missouri, and all experimental manipulations were performed under an approved University of Missouri Animal Care and Use protocol.

\section{Exercise regimen}

Four week old male and female WT and oim/oim mice were randomly divided into swimming exercise, volunteer wheel running, or control (non-exercise in-cage activity) groups. At six weeks of age, mice in the swimming exercise group were acclimated to the swimming exercise protocol in the first 3 weeks as described in Table II-S1, and then full 60 minutes ( 3 sessions of 20 minutes of swimming exercise with 3 minutes of rest in between each session) of swimming exercise was maintained. Four to five week old mice in the volunteer wheel running group were housed individually in cages equipped with a running wheel [Kaytee Silent Spinner, 4.5inch diameter,136g (wheel weight alone; 75g), 
Kaytee Products, Inc, Walnut Creek, CA] assembled with cyclocomputer (SpeedZone ${ }^{\circledR}$ Comp, Specialized Bicycle Components Inc., Morgan Hill, CA) for recording max speed $(\mathrm{km} / \mathrm{h})$, average speed $(\mathrm{km} / \mathrm{h})$, distance $(\mathrm{km})$, running time $(\mathrm{hr})$, and odometer reading $(\mathrm{km})$ daily. Mice were monitored daily for evidence of adverse effects of exercise [injury, lameness, weight loss, poor grooming, and change in behavior (major change in time on the wheel)]. At 4 months of age, mice were euthanized and hindlimb muscles and femurs evaluated.

\section{Contractile Properties}

Contractile properties of the soleus (sol), plantaris (plant), gastrocnemius (gast), and tibialis anterior (TA) muscles in male and female WT and oim/oim mice were evaluated as previously described. Briefly, mice were anesthetized and the left sol, plant, gast, and TA muscle surgically exposed at their distal insertions. The distal tendon of each muscle was attached to the Grass force transducer and sequentially tested, sol $\rightarrow$ plant $\rightarrow$ gast $\rightarrow$ TA. The distal tendon was adjusted in length so that the passive tension was zero grams. The sciatic nerve was isolated and placed on a stimulating electrode and a twitch was obtained as previously described[3]. At optimal length, a peak tetanic contraction $\left(\mathrm{P}_{\mathrm{o}}\right)$ was elicited by pulses delivered at $150 \mathrm{~Hz}, 300-\mathrm{ms}$ duration, and an intensity of 6V for each type muscle[225]. Previous studies of force curves generated at $15,50,75,100$ and $125 \mathrm{~Hz}(6 \mathrm{~V}, 300 \mathrm{~ms})$ demonstrated that all the muscles were

maximally recruited by the time $100 \mathrm{~Hz}$ was reached[3]. All data were collected using PowerLab® (ADinstruments, Colorado Springs, CO). 


\section{Femoral Geometry and Torsional Loading to Failure}

Right femora were evaluated by $\mu \mathrm{CT}$ scan analysis [Siemens Inveon $\mu \mathrm{CT}$ equipped with Siemens Inveon Acquisition Workplace Software Version 1.5 (Siemens Preclinical Solutions, Knoxville) with an X-ray peak of $80 \mathrm{kVp}$ and an exposure time of 140ms] prior to ex vivo torsional loading to failure analyses as previously described[224]. $\mu \mathrm{CT}$ image slices were analyzed using the Amira 5.3.3 software package (Mercury Computer Systems/TGS, Chelmsford, MA) to give a cubic voxel dimension of $0.083 \mathrm{~mm}^{3}$. The mid-shaft slice located and modeled as a hollow elliptical cross-section. The marrow cavity diameter (MCD) reported as the average of the two endosteal diameters, the cortical bone width (CBW) reported as the average of the four thicknesses on the major and minor axes of the cortical mid-slice, and the polar moment of area $\left(\mathrm{K}: \mathrm{mm}^{4}\right)$ determined as previously described. Following $\mu \mathrm{CT}$ analyses, the femora were potted into individualized cylindrical holders and the torsional loading to failure evaluated using the TA-HDi testing machine (Stable Micro Systems, Surrey, UK)[224]. Applied torque T $(\mathrm{Nmm})$ was calculated and plotted as a function of relative angular displacement $\theta$ (degrees). The whole bone parameters of strength [torsional ultimate strength $\left(\mathrm{T}_{\max }\right.$, $\mathrm{Nmm}$ ) and strain energy to failure (U, Nmm)] and stiffness [torsional stiffness (Ks, $\mathrm{Nmm} / \mathrm{rad}$ ), which take into account bone geometry and material properties, were determined as previously described[224]. The bone material properties, tensile strength $\left(\mathrm{Su}, \mathrm{N} / \mathrm{mm}^{2}\right)$ and stiffness [shear modulus of elasticity, $\left(\mathrm{N} / \mathrm{mm}^{2}\right)$ ] were also determined according to Carleton et al[224]. 


\section{Western Blot Analysis}

Western blots were performed using a modified version of a previously described protocol[226]. Briefly, right gastrocnemius muscle, previously stored at $-80^{\circ} \mathrm{C}$, was homogenized in lysis buffer. Protein was mixed with 6x Laemmli buffer, boiled, and loaded on a Criterion TGX Pre-cast gel (Bio-Rad, Hercules, CA). After size separation via gel electrophoresis, the proteins were transferred to PDVF membrane. $5 \%$ milk in tris-buffered saline with Tween 20 (TBST) was used for blocking. The blots were labeled with primary antibodies against the total OXPHOS Antibody Cocktail (Abcam, Cambridge, MA, ab10413) containing 5 mouse monoclonal antibodies [CI subunit NDUFB8 (ab110242), CII-30kDa (ab14714), CIII-Core protein 2 (ab14745) CIV subunit I (ab14705) and CV alpha subunit (ab14748)], TFAM (Abcam, Cambridge, MA, ab89818), PGC-1 $\alpha$ (MilliporeSigma, Burlington, MA, 516557), BNIP3 (Cell Signaling, Danvers, MA, 3769), PINK1 (Cell Signaling, Danvers, MA, 6946), Parkin (Cell Signaling, Danvers, MA, 4211S), P62 (Cell Signaling, Danvers, MA, 5114), and LC3 A/B (Cell Signaling, Danvers, MA, 12741). Primary antibodies were diluted to 1:1,000 in

5\% BSA in TBST. Secondary horseradish peroxidase-conjugated antibodies were diluted to $1: 5,000$ in 5\% milk in TBST. Blots were imaged and analyzed using Image Lab ${ }^{\mathrm{TM}}$ Software (Bio-Rad, Hercules, CA); protein content was normalized using amido black total protein stain (Figure II-S4).

\section{Mitochondria Isolation and Respiration}

Mitochondrial isolation and high resolution respirometry were carried out using modified versions of previously described methods[227-229]. For mitochondrial isolation, both 
right and left gastrocnemius were removed, cleaned of extraneous fat and tissue, and placed in mitochondrial isolation buffer $(100 \mathrm{mM} \mathrm{KCl}, 50 \mathrm{mM} \mathrm{MOPS}, 5 \mathrm{mM} \mathrm{MgSO} 4,1$ mM EGTA, and $1 \mathrm{mM}$ ATP), on ice. Gastrocnemius muscles were homogenized using a rotor type homogenizer, the homogenate was centrifuged $\left(800 \mathrm{xg}, 4^{\circ} \mathrm{C}, 10 \mathrm{~min}\right)$, and the supernatant filtered through gauze. The pellet was re-suspended in $5 \mathrm{~mL}$ mitochondrial isolation buffer, homogenized using a Potter-Elvehjenm homogenizer (Teflon on glass), and centrifuged $\left(800 \mathrm{xg}, 4^{\circ} \mathrm{C}, 10 \mathrm{~min}\right)$. The supernatant was filtered, added to the previously filtered supernatant, and centrifuged $\left(12,000 \mathrm{xg}, 4^{\circ} \mathrm{C}, 10 \mathrm{~min}\right)$. The pellet was re-suspended in $3 \mathrm{~mL}$ isolation buffer with glass on glass homogenization, centrifuged $\left(8,000 \mathrm{xg}, 4^{\circ} \mathrm{C}, 10 \mathrm{~min}\right)$, and the process repeated with $2 \mathrm{~mL}$ isolation buffer. $0.2 \%$ Bovine Serum Albumin (BSA) was added to $2 \mathrm{~mL}$ of the isolation buffer and used to resuspend the pellet a third time via glass on glass homogenization followed by centrifugation $\left(8,000 \mathrm{xg}, 4^{\circ} \mathrm{C}, 10 \mathrm{~min}\right)$. Finally, the pellet was re-suspended in $200 \mu \mathrm{L}$ $\mathrm{MiPO}_{3}$ buffer (0.5 mM EGTA, $3 \mathrm{mM} \mathrm{MgCl2} \cdot 6 \mathrm{H} 20$, $60 \mathrm{mM}$ K-lactobionate, $20 \mathrm{mM}$ Taurine, 10 mM KH2P04, 20 mM HEPES, 110 mM Sucrose, 1g/l BSA, 20 mM Histidine, $20 \mu \mathrm{M}$ vitamin E succinate, $3 \mathrm{mM}$ glutathione, $1 \mu \mathrm{M}$ leupeptine, $2 \mathrm{mM}$ glutamate, $2 \mathrm{mM}$ malate, $2 \mathrm{mM} \mathrm{Mg}$-ATP)[227] and allowed to equilibrate on ice for 30 $\min$.

High resolution respirometry was used to measure mitochondrial respiration by the isolated mitochondria using the Orosboros Oxygraph-2k (Orosboros Instruments; Innsbruck, Austria). $30-45 \mu 1$ of isolated mitochondria were added to the respiration chambers to measure basal respiration. Steady-state oxygen flux was measured by the addition of $2 \mathrm{mM}$ malate and $5 \mathrm{mM}$ glutamate. Oxygen flux through complex I was 
measured by the titration of $125-375 \mu \mathrm{M}$ of ADP; through complex I+II by the titration of 1-5 mM succinate, and maximal uncoupled respiration was measured by the addition of $0.125 \mu \mathrm{M}$ FCCP. Finally, $10 \mu \mathrm{M}$ cytochrome $\mathrm{C}$ was added to assess the quality of mitochondrial isolation. All measurements were normalized to the mitochondrial protein content.

\section{Transmission Electron Microscopy and Analysis}

Soleus muscle was excised, cleaned of any extraneous tissue or fat and placed in glutaraldehyde fixative at room temperature for 1 hour before being transferred to $4{ }^{\circ} \mathrm{C}$. Tissue was processed and prepared by the University of Missouri Electron Microscopy Core. Samples were imaged using a JEOL JEM-1400 120kV TEM. Images were captured at a magnification of 3,000X. Analysis was done using classical stereological methods as previously described to calculate the following, intermyofibrillar mitochondria: mitochondrial volume density, cross sectional area, and numerical density using the low magnification images[230,231].

\section{Mitochondrial DNA Content}

Mitochondrial DNA content was measured as a ratio of mitochondrial DNA to nuclear DNA using quantitative PCR (qPCR) methods and primers described by Malik et al[232]. Briefly, DNA was isolated from gastrocnemius muscle using a DNeasy Blood and Tissue Kit (Qiagen, Germantown, MD). qPCR was carried out in $10 \mu 1$ reaction volume containing $5 \mu 1$ PowerUp SYBR green master mix, $0.5 \mu 1$ forward primer, $0.5 \mu 1$ reverse primer, and $4 \mu \mathrm{l}$ of diluted DNA. The mitochondrial DNA forward and reverse primers used were mMitoF1 (5'-CTAGAAACCCCGAAACCAAA-3') and mMitoR1 (5'- 
CCAGCTATCACCAAGCTCGT-3'), respectively, and the nuclear DNA forward and reverse primers used were mB2MF1 (5'-ATGGGAAGCCGAACATACTG-3') and mB2MR1 (5'-CAGTCTCAGTGGGGGTGAATA-3'), respectively. A standard curve with copy numbers ranging from $10^{2}-10^{9}$ was amplified simultaneously with the isolated DNA used to quantify the ratio of mtDNA to nuclear DNA.

\section{Citrate synthase activity}

Citrate synthase activity was measured in mitochondria isolated from mixed gastrocnemius muscle using methods by Srere et al.[233], as previously described[226].

\section{Glycogen Content}

Snap frozen gastrocnemius muscle was powdered using a mortar and pestle on liquid nitrogen and approximately $15-30 \mathrm{mg}$ of powdered muscle was added to $0.5 \mathrm{~mL}$ of $1 \mathrm{~N}$ HCL. The muscle was placed in a boiling water bath for 2.5 hours. After boiling, tubes were vortexed, and $1.5 \mathrm{~mL}$ of $0.67 \mathrm{M} \mathrm{NaOH}$ was added. The acid hydrolysis converts glycogen to free glucose which was then measured using Infinity Glucose Hexokinase Liquid Stable Reagent (Thermo Fischer Scientific, Waltham, MA) according to the manufacturer protocol using a sample to reagent ratio of 22.5:1[234]. 


\section{Statistical Analysis}

Statistical analyses were performed using SAS (SAS Institute Inc., Cary, NC). Swimming and voluntary wheel running exercise regimens were analyzed separately as a $2 \mathrm{X} 2 \mathrm{X} 2$ factorial [2 genotypes (WT and oim/oim) X 2 sexes X 2 treatments (control and exercise) and mitochondrial analyses were analyzed as $2 \mathrm{X} 2$ factorials [2 genotypes (WT and oim/oim) X 2 sexes] using Fisher's Protected Least Significant Difference[235,236]. If heterogeneous variations were present, a log transformation was used to stabilize the variation. If the log transformation failed to stabilize the variation, a nonparametric ranked analysis was performed according to Conover et al[237]. Differences were considered significant at $p \leq 0.05$.

\section{Results}

\section{Effect of volunteer wheel running and swimming exercise on muscle weight and} function

As was demonstrated in our previous study[3], oim/oim mice exhibited reduced hindlimb muscle weights and absolute contractile forces as compared to WT littermates (Figure II1), with minimal impact on relative contractile forces. Previously the decreased absolute contractile forces in male oim/oim hindlimb muscles was directly associated with decreased specific contractile force in male oim/oim plantaris (plant), gastrocnemius (gast), and tibialis anterior (TA) muscles, even though relative contractile forces did not always reach significance[3,163]. Volunteer wheel running and swimming exercised male oim/oim mice exhibited decreases in plant, gast, and TA muscle wet weight when compared to in-cage control counterparts (Figure II-1 B-D). Peak tetanic force of plant, 
gast, and TA muscle in volunteer wheel running exercised and plant and TA muscle in swimming exercised male oim/oim mice were reduced as compared to their in-cage control counterparts (Figure II-1F-H). These decreases were still evident in the relative contractile force [peak tetanic force $\left(\mathrm{P}_{\mathrm{o}} ; \mathrm{g}\right) /$ muscle weight $\left.(\mathrm{mg})\right]$ of volunteer wheel running exercised male oim/oim plant and gast muscles, and swimming exercised male oim/oim plant muscle (Figure II-1J\&K). However, interestingly, female mice did not exhibit any changes in muscle weight or function with both type of exercises regardless of genotypes (Figure II-S1). Both male and female oim/oim mice ran shorter total distances, $42 \%$ and $35 \%$ of the total distances ran by WT littermates, respectively (Figure II-S2). The average speed $(\mathrm{km} / \mathrm{hr})$ ran per week was also reduced in male and female oim/oim mice being $57 \%$ and $63 \%$ of WT speeds, respectively. In addition to reduced distance and average speed, the average time on the wheel (minutes) per week spent by oim/oim was $37 \%$ and $51 \%$ less that WT mice for both males $(\mathrm{p}=0.122)$ and females, respectively (Figure II-S2). 

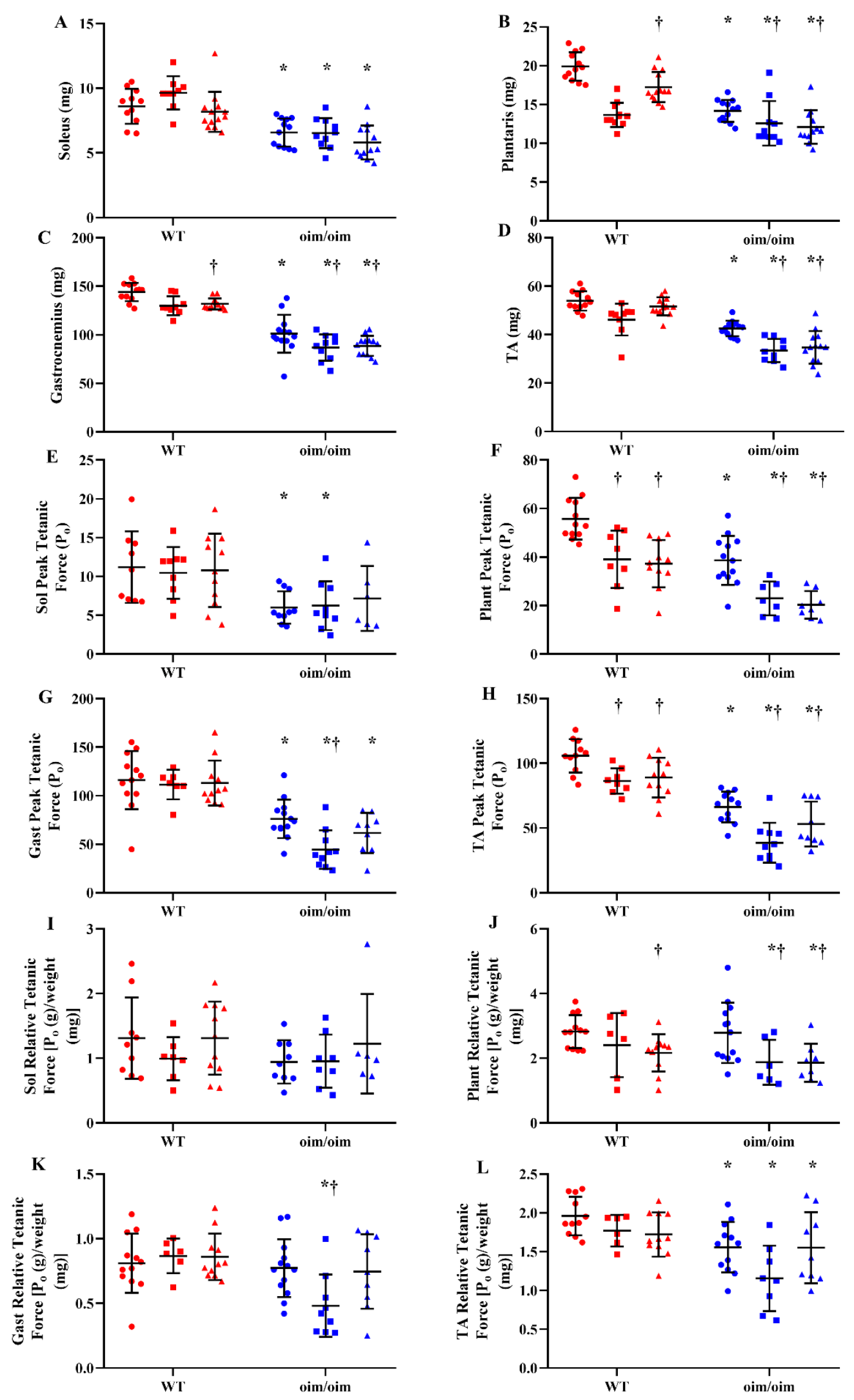
Figure II-1: Volunteer wheel running and swimming exercised male oim/oim mice had reduced hindlimb skeletal muscle weight and peak tetanic force as compared to in-cage control counterparts. (A) Soleus, (B), Plantaris, (C) Gastrocnemius, and (D) Tibialis Anterior muscle wet weights. (E) Soleus peak tetanic force $\left(\mathrm{P}_{\mathrm{o}}\right)$, (F) Plantaris Peak $\mathrm{P}_{\mathrm{o}},(\mathrm{G})$ Gastrocnemius Peak $\mathrm{P}_{\mathrm{o}},(\mathrm{H})$ Tibialis Anterior Peak $\mathrm{P}_{0}$, (I) Soleus relative $\mathrm{P}_{\mathrm{o}}$, (J) Plantaris relative $\mathrm{P}_{\mathrm{o}},(\mathrm{K})$ Gastrocnemius relative $\mathrm{P}_{\mathrm{o}}$, and (L) Tibialis Anterior relative $\mathrm{P}_{\mathrm{o}}$ of 4-month-old male WT and oim/oim in-cage control, volunteer wheel running, and swimming exercised mice. Graphs are presented as MEAN \pm STDEV. ${ }^{*} \mathrm{p} \leq 0.05$ vs. WT; $\dagger \mathrm{p} \leq 0.05$ vs. in-cage control. ( $\mathrm{n}=6-13$ per group). Red and blue symbols represent WT and oim/oim, respectively. Circles, squares, and triangles represent control, wheel running, and swimming, respectively. 


\section{Effect of volunteer wheel running and swimming exercise on skeletal properties}

Similar to previous investigations, oim/oim mice exhibit compromised bone microarchitecture and biomechanical properties as compared to their WT littermates regardless of sex and exercise regimen (Tables II-S2\&S3, Figure II-2 A-D). Male WT mice that underwent volunteer wheel running exercise demonstrated decreases in several geometric and biomechanical parameters, including cortical bone width (CBW), polar moment of area $(\mathrm{K})$, torsional ultimate strength $\left(\mathrm{T}_{\max }\right)$, torsional stiffness $(\mathrm{Ks})$, and energy to failure (U), while shear modulus of elasticity increased as compared to in-cage control counterparts (Figure II-2 A \& C and Table II-S2). Swimming exercised male WT mice also showed decreases in $\mathrm{CBW}$ and $\mathrm{K}$ and increase in marrow cavity diameter (Table II-S2). Male oim/oim and female WT and oim/oim mice did not exhibit any changes in bone geometric or biomechanical properties with either of exercise regimen; except that female WT mice had decreases in $\mathrm{T}_{\max }$ and tensile strength $(\mathrm{Su})$ and female oim/oim mice had decreased in $\mathrm{T}_{\max }$ with swimming exercise (Figure II-2 B and D and Table II-S3). Pearson's correlation coefficients were evaluated between total distances run and bone biomechanical parameters in WT and oim/oim mice. The total distances run by male and female WT mice exhibited positive correlations with $\mathrm{T}_{\max }(+0.508 ; p=0.022)$ and $\mathrm{U}(+0.500 ; p=0.025)$, while oim/oim mice did not (Figure II-2E-H). The lack of positive correlations in oim/oim mice suggest that $\mathrm{oim} / \mathrm{oim}$ mice are not able to respond as WT mice to weight bearing exercise, leading us to further question the musculoskeletal function of oim/oim. 

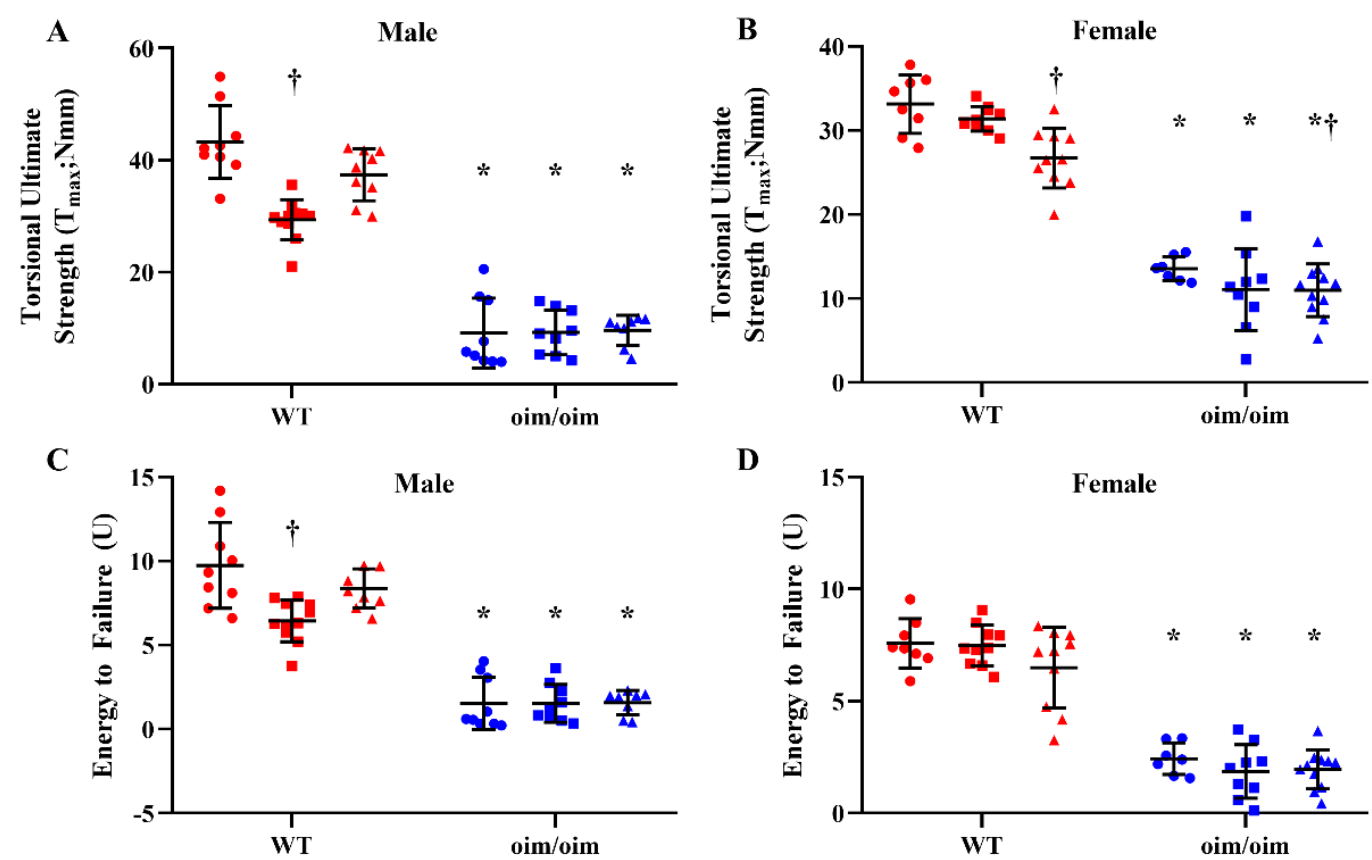

D
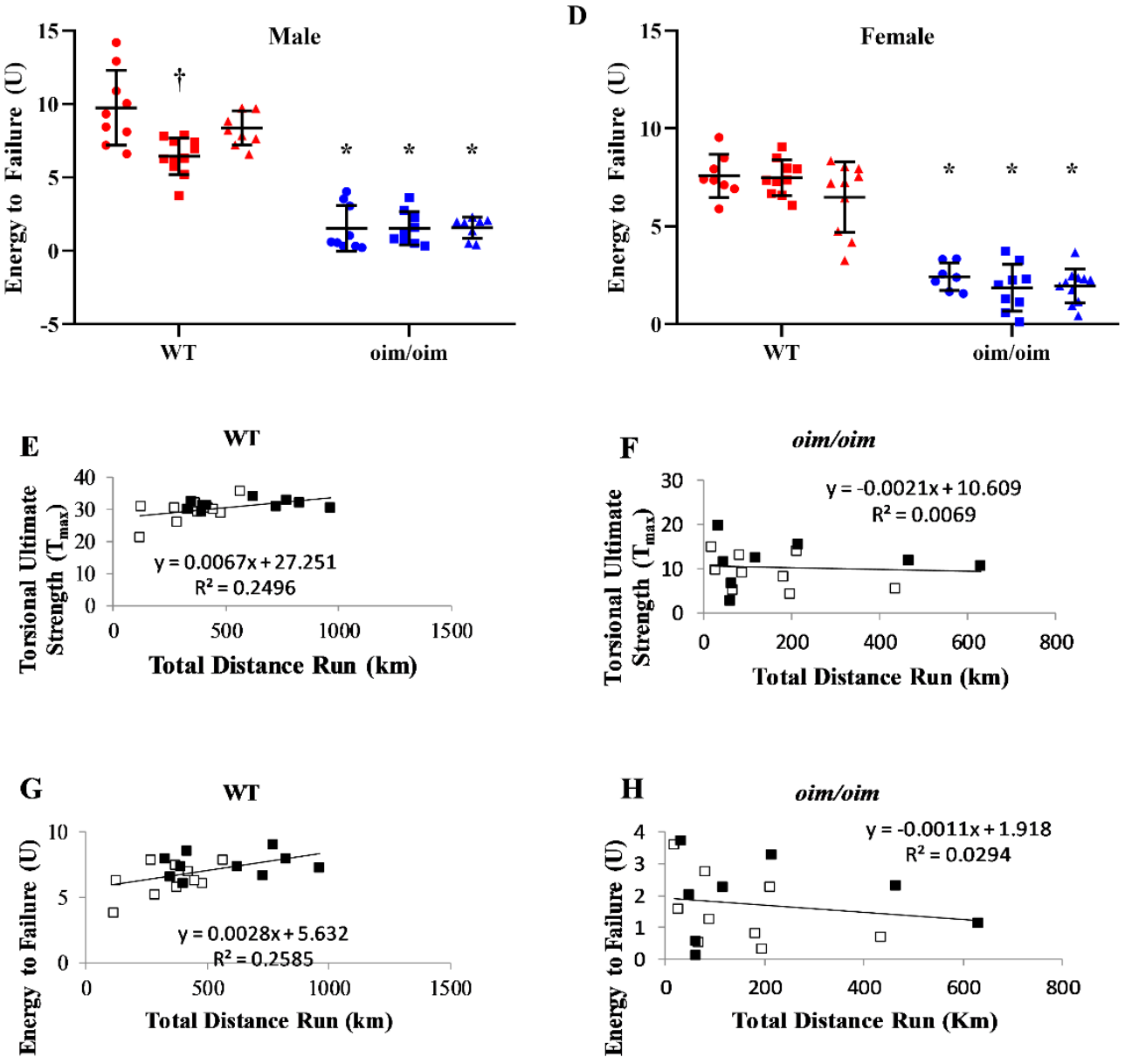
Figure II-2: Oim/oim mice exhibited compromised femoral biomechanical properties as compared to WT littermates. Volunteer wheel running exercised WT mice showed positive correlation between biomechanical properties and the total distance ran $(\mathrm{km})$, but oim/oim mice did not. Torsional ultimate strength $\left(\mathrm{T}_{\max }\right)$ of (A) male and (B) female mice; energy to failure (U) of (C) male and (D) female mice. Red and blue symbols represent WT and oim/oim, respectively. Circles, squares, and triangles represent control, wheel running, and swimming, respectively.

Linear regression analysis of the correlation between $\mathrm{T}_{\max }$ and total distance run by (E) WT and (F) oim/oim mice, linear regression analysis of the correlation between $\mathrm{U}$ and total distance run of $(\mathrm{G}) \mathrm{WT}$ and $(\mathrm{H})$ oim/oim mice. Graphs are presented as MEAN \pm STDEV. ${ }^{*} p \leq 0.05$ vs. WT; $\uparrow p \leq 0.05$ vs. in-cage control. $(\mathrm{n}=7-13$ per group). White and black squares represent male and female animals, respectively. 


\section{Protein levels of electron transport chain (ETC) complexes}

Previously, our group determined that citrate synthase activity was increased in oim/oim mixed gastrocnemius muscle homogenates relative to WT mixed gastrocnemius muscle homogenates[163], which in light of the response of oim/oim mice to the exercise regimens, prompted the evaluation of the ETC complexes in the current study. Oim/oim mice exhibited reduced levels of ETC complex IV, mitochondria encoded cytochrome oxidase I [ $51 \%$ decrease in females and $20 \%$ decrease (which did not reach significance) in males] as compared to WT counterparts (Figure II-3), while the protein levels of other complexes did not differ between genotypes. 
A
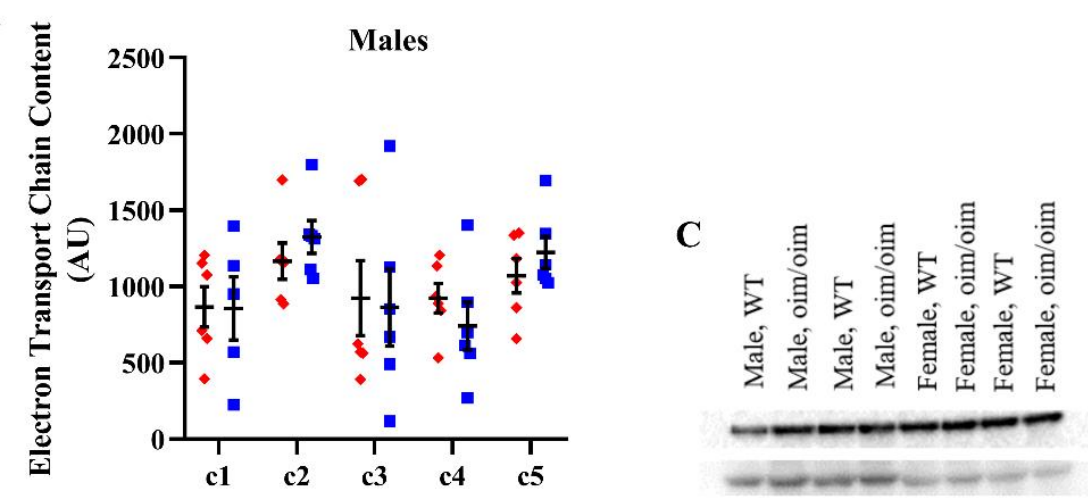

Complex V

Complex III

Complex IV

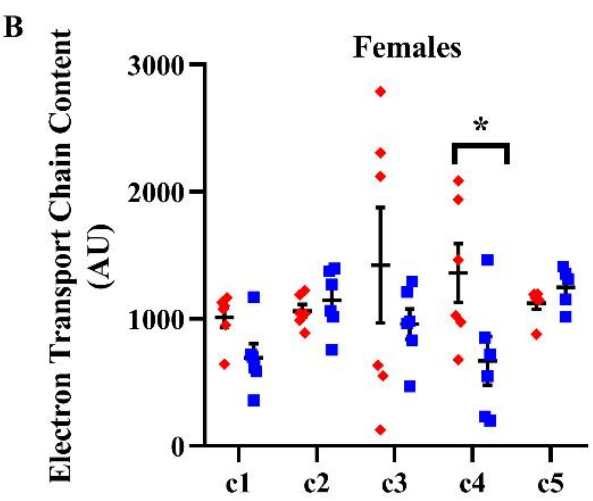

Complex II

Complex I

Figure II-3: Female oim/oim mixed gastrocnemius muscle homogenate had reduced ETC complex IV mitochondrial encoded cytochrome c oxidase I (c4). Western blot analysis of electron transport chain (ETC) complexes measured in 4month-old mixed gastrocnemius muscle of (A) male and (B) female WT (red diamonds) and oim/oim (blue squares) mice. (C) Representative blot images. Graphs are presented as MEANS \pm SE. ${ }^{*} \mathrm{p} \leq 0.05$ vs. WT. ( $\mathrm{n}=5-6$ per group). 


\section{Skeletal Muscle Mitochondrial Function}

To investigate skeletal muscle mitochondrial function more directly, mitochondria were isolated from 4-month-old male WT and oim/oim mice mixed gastrocnemius muscles, and oxygen consumption was measured using high resolution respirometry. Oim/oim skeletal muscle mitochondria demonstrated reductions in respiration during basal (-65\%), state $2(-52 \%)$, state 3-Complex I (-61\%), state 3-Complex I+II (-53\%), and maximal uncoupled respiration (-52\%) as compared to WT skeletal muscle mitochondria (Figure II-4A). These decreases clearly demonstrate inefficient mitochondrial function with compromised energy production in oim/oim gastrocnemius muscle.

In addition, the isolated oim/oim gastrocnemius muscle mitochondria exhibited a $33 \%$ decrease in citrate synthase activity as compared to WT gastrocnemius muscle

mitochondria (Figure II-4B). While citrate synthase activity in whole muscle homogenate is often an indirect marker of mitochondrial content/mass, in isolated mitochondria, citrate synthase activity can provide insight into mitochondrial function[238-240]. 

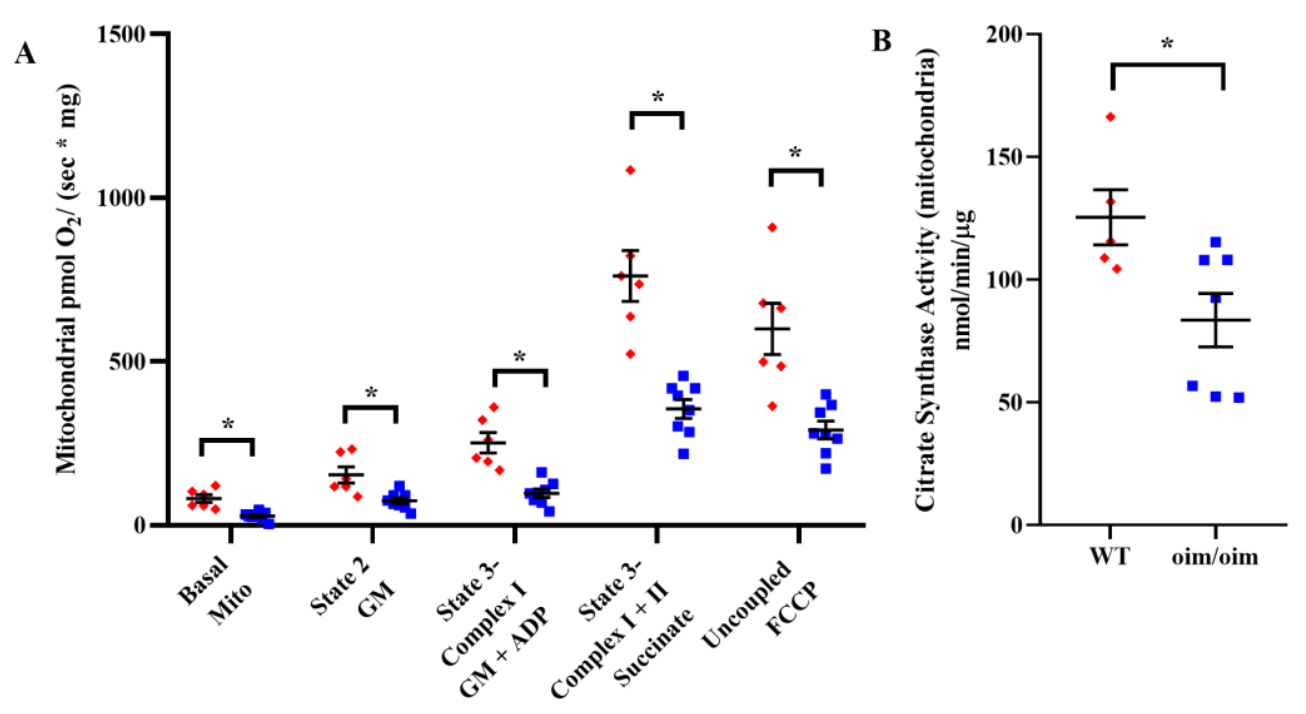

Figure II-4: (A) Male oim/oim mice exhibit reduced mitochondrial respiration and (B) mitochondrial citrate synthase activity compared to WT littermates. (A) Basal, state 2, state 3, and maximal uncoupled mitochondrial respiration was significantly decreased in 4-month-old oim/oim (blue squares) male mice compared to WT (red diamonds) littermates. Oxygen consumption was measured with the addition of substrates to stimulate different respiration states: basal (mitochondria in $\mathrm{MiPO}_{3}$ buffer), state 2 (Glutamate and Malate (GM)), state 3 respiration with electron flux through complex I (GM and ADP), state 3 respiration with electron flux through complex I and II (succinate), and maximal uncoupled respiration (FCCP). (B) Citrate synthase activity was decreased in isolated mitochondria from 4-month-old male oim/oim mice compared to WT littermates. Graphs are presented as Means $\pm \mathrm{SE}$ of the genotype values. ${ }^{*} \mathrm{p} \leq 0.05$ vs. WT. ( $\mathrm{n}=5-8$ per group). 


\section{Mitochondrial Biogenesis and Mitophagy}

To determine if oim/oim mice have altered mitochondrial turnover, markers of mitochondrial biogenesis and autophagy/mitophagy were evaluated. Mitochondrial biogenesis markers, PGC1- $\alpha$ (a nuclear co-activator) and TFAM (a mitochondrial transcription factor)[187] were increased in male oim/oim mixed gastrocnemius muscle while TFAM was increased in female oim/oim mixed gastrocnemius muscle compared to their WT counterparts (Figure II-5A, Supplemental Figure II-3A). Several markers of autophagy and mitophagy were also evaluated including LC3, a known component of autophagosome formation, as well as the LC3-II to LC3-I ratio, a method to assess autophagy; with increases in the LC3-II/I ratio being suggestive of elevated autophagy[186,187,241,242] and warranting direct studies of autophagy flux. In the skeletal muscle of male oim/oim mixed gastrocnemius, the LC3-II/I ratio was reduced by greater than 50\% compared to WT counterparts; a decrease largely driven by increases in LC3-I (117\%)(Figure II-5B \& C). The levels of other autophagy/mitophagy markers including PINK1, Parkin, BNIP3, and p62 did not differ between genotypes. 


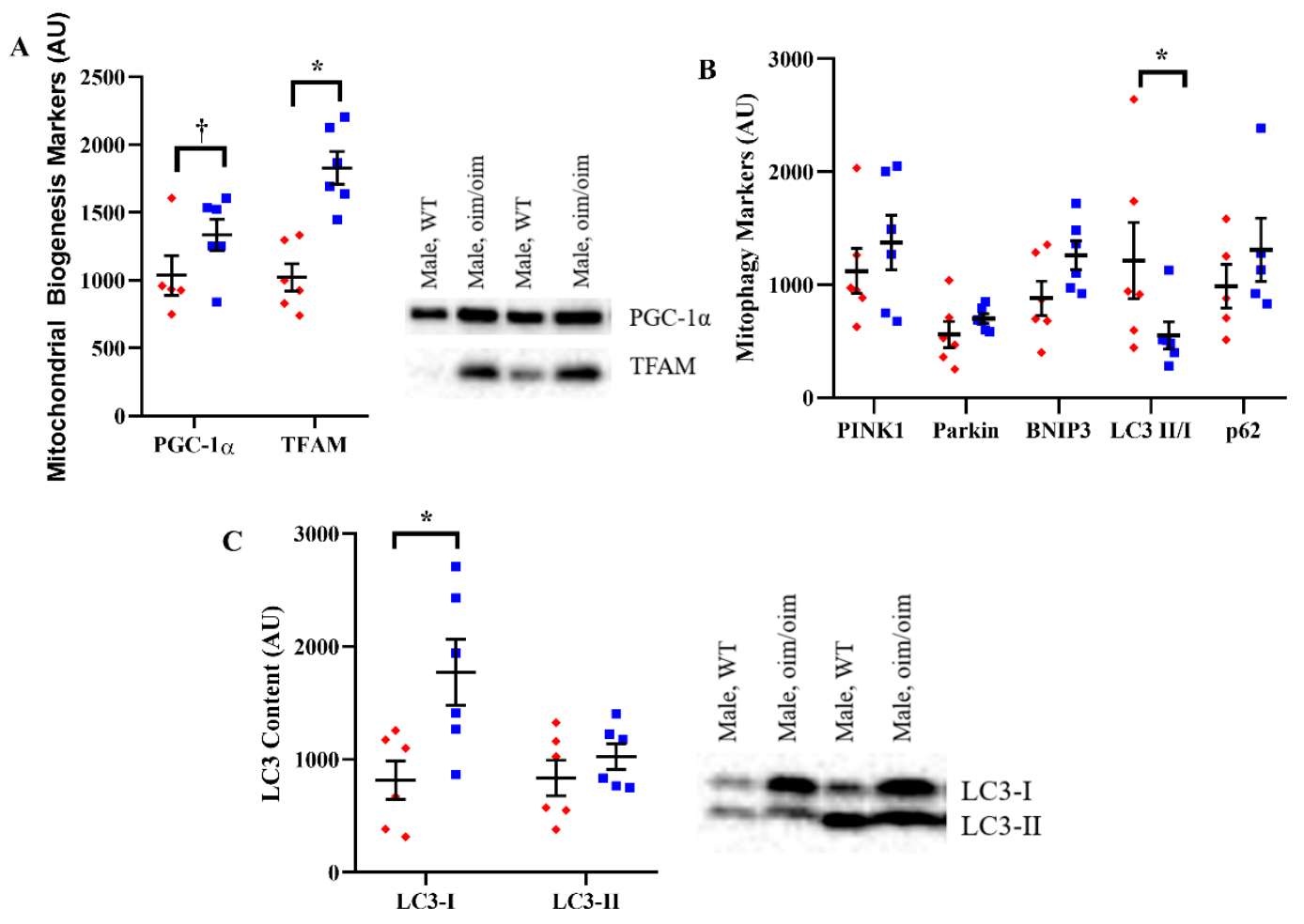

Figure II-5: Male oim/oim mice exhibit increased markers of mitochondrial biogenesis and decreased markers of autophagy and mitophagy. Western blot analysis of markers of (A) mitochondrial biogenesis (PGC- $1 \alpha$ and TFAM) and (B) autophagy and mitophagy (PINK1, Parkin, BNIP3, LC3 II/I, and p62) in protein homogenate isolated from 4-month-old male oim/oim mixed gastrocnemius muscle. (A) TFAM and PGC- $1 \alpha(\mathrm{p}=0.07)$, a marker of mitochondrial biogenesis was increased in oim/oim (blue squares) mice compared to WT (red diamonds) mice and (B) LC3 II/I, a marker of mitophagy, was decreased in oim/oim mice compared to WT mice driven by an increase in (C) LC3-I. Graphs are presented as MEANS \pm SE. ${ }^{*} p \leq 0.05$ vs. WT; $\uparrow p=0.07$ vs WT. $(n=5-6$ per group). 
To determine if the decrease in the LC3-II/I ratio seen in the whole gastrocnemius muscle homogenate was indicative of decreased mitochondrial degradation rather than decreases in general autophagy, we measured LC3-II and BNIP3 levels in isolated mitochondria of mixed gastrocnemius muscle and found that LC3-II levels in the 4-month-old male oim/oim mice were dramatically decreased compared to their WT counterparts while BNIP3 levels appeared reduced but did not reach significance, further suggesting that mitophagy is decreased in the skeletal muscle of oim/oim mice (Figure II-6).

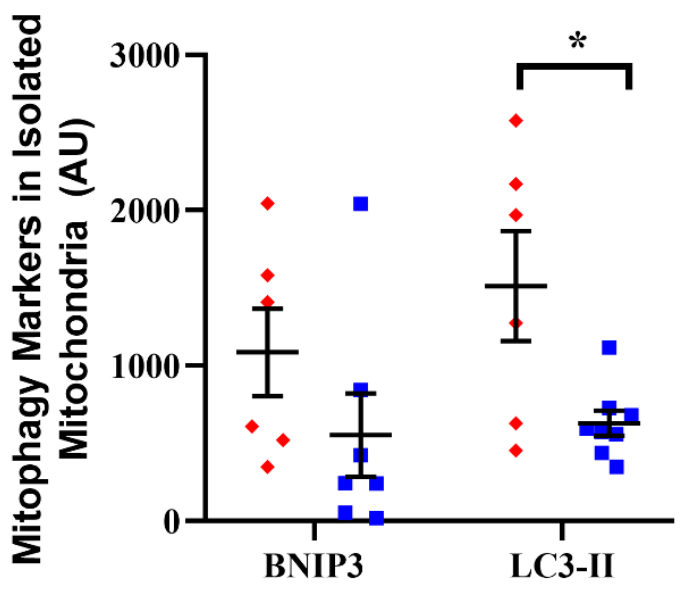

Figure II-6: BNIP3 and LC3-II levels were measured in isolated mitochondria of 4-month-old male WT (red diamonds) and oim/oim (blue squares) mixed gastrocnemius muscle. LC3-II levels were significantly reduced in oim/oim mitochondrial compared to WT mitochondria. Graphs are presented as MEANS \pm SE * $\mathrm{p} \leq 0.05$ vs WT. ( $\mathrm{n}=6-8$ per group). 


\section{Mitochondrial Content}

Electron microscopic analyses of 4-month-old male oim/oim and WT soleus muscle found no evidence of gross abnormal morphological changes in oim/oim soleus muscle. In addition no differences were found in intermyofibrillar mitochondrial volume density, cross-sectional area, and numerical density (Figure II-7). To investigate further, the relative mitochondrial DNA content was evaluated by the ratio of mitochondrial DNA to nuclear DNA in 4-month-old male and female oim/oim and WT gastrocnemius muscles. The male and female oim/oim gastrocnemius mitochondrial/nuclear DNA ratios were decreased by $40 \%$ and $34 \%$ relative to the mitochondrial/nuclear DNA ratios of sex matched WT gastrocnemius muscles (although not reaching significance), respectively (Figure II-8). 
A

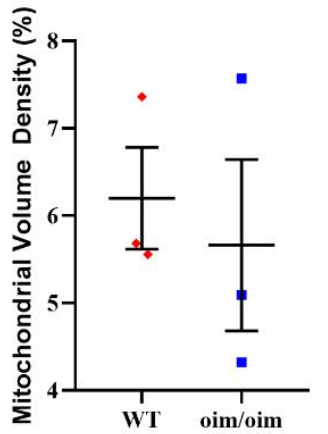

B

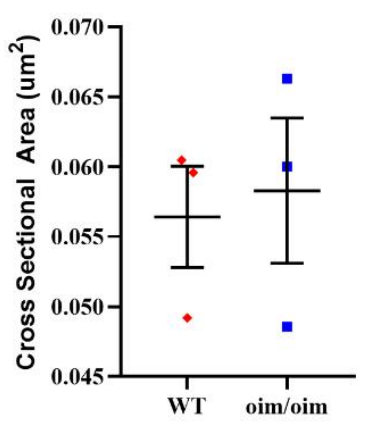

C

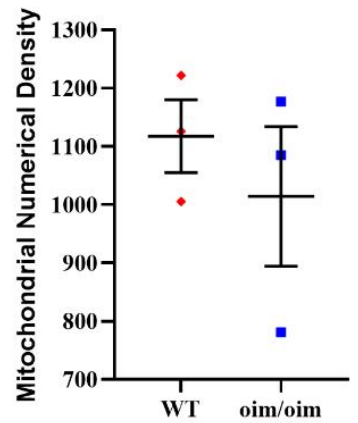

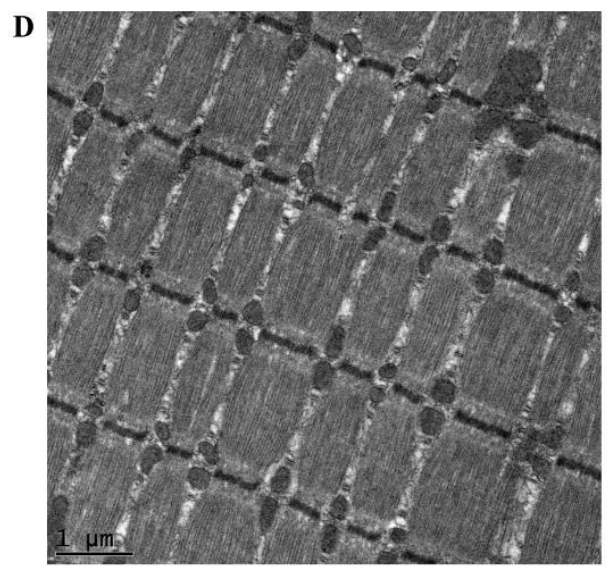

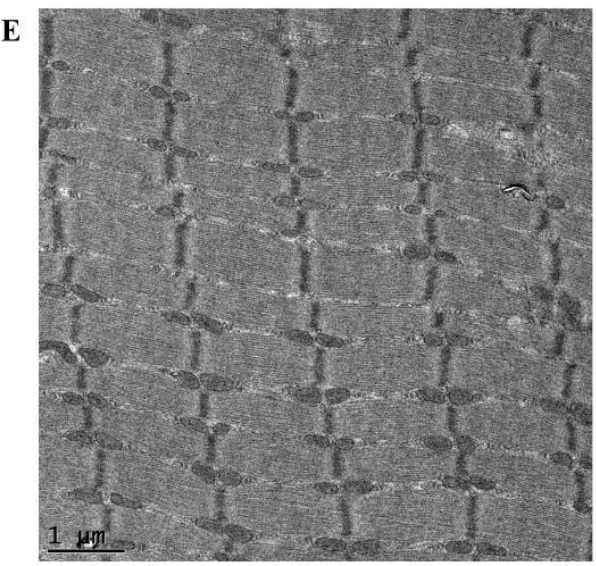

Figure II-7: Ten representative images of 4-month-old WT (red diamonds) and oim/oim (blue squares) intermyofibrillar mitochondria from soleus muscle were assessed using Transmission Electron Microscopy and classical stereological methods. The intermyofibrillar (A) mitochondria volume density, (B) cross sectional area, and (C) mitochondrial numerical density as well as gross morphology were not different between (D) WT and (E) oim/oim soleus muscle. Graphs are presented as MEANS \pm SE. ( $n=3$ per group). 


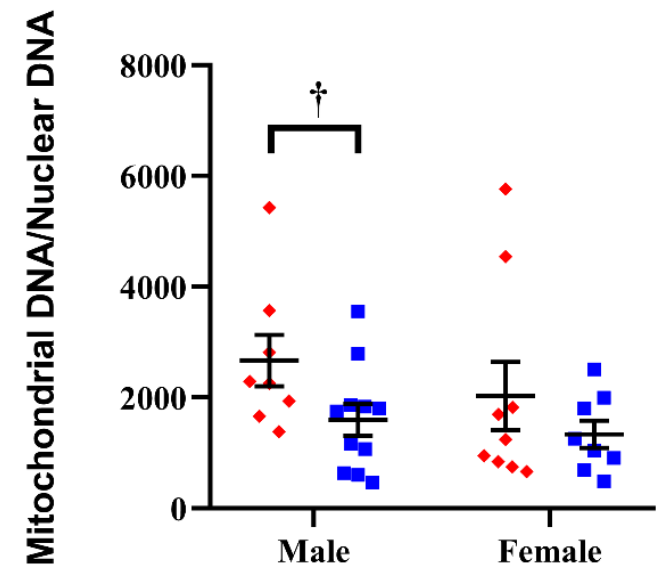

Figure II-8: Oim/oim mice exhibit a reduced mitochondrial DNA to nuclear DNA ratio. The ratio of mitochondrial DNA copy number to nuclear DNA copy number was decreased by $40 \%$ in (A) male and by $34 \%$ in (B) female 4 -monthold oim/oim (blue squares) mixed gastrocnemius muscle as compared to WT (red diamonds) mixed gastrocnemius muscle. Graphs are presented as MEANS \pm SE. $\dagger \mathrm{p}=0.08$ vs. WT. $(\mathrm{n}=8-11$ per group) 


\section{Skeletal Muscle Glycogen Content}

Effective glycogen metabolism provides glucose to muscle cells to induce muscle contraction, and alterations could result in reduced muscle contractile forces. To investigate glycogen metabolism, skeletal muscle glycogen content was evaluated in WT and oim/oim gastrocnemius muscles. No differences between WT and oim/oim mice were found, further suggesting that the mitochondrial dysfunction itself is likely the major contributor to the reduced contractile force in oim/oim mice (Figure II-9).

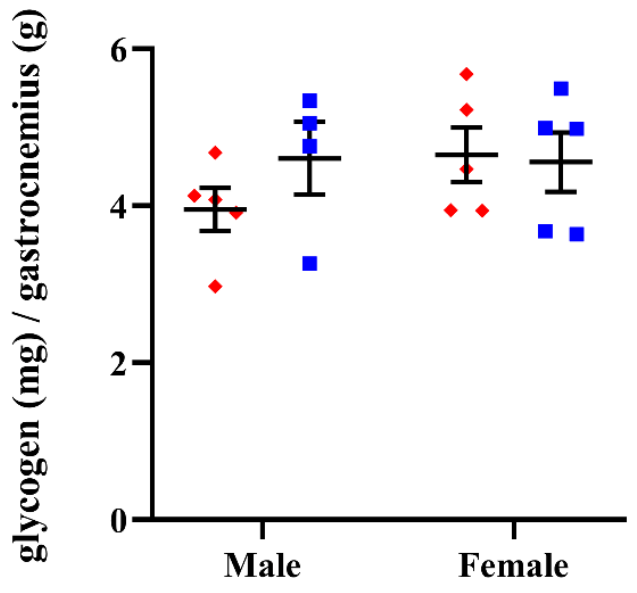

Figure II-9: Glycogen content is equivalent in oim/oim (blue squares) relative to WT (red diamonds) mixed gastrocnemius muscle. Glycogen content was measured in gastrocnemius muscle of 4-month-old (A) male and (B) female oim/oim and WT. Graphs are presented as MEANS \pm SE. ( $\mathrm{n}=4-5$ per group). 


\section{Discussion}

The beneficial impact of exercise on overall health is well established, and particularly its positive impact on musculoskeletal health during the pre-pubertal/pubertal growth period[115,126,129,211,212]. We chose to test the impact of two different exercise modalities, voluntary wheel running (weight-bearing) and swimming (non-weight bearing)[243] on WT and oim/oim hindlimb muscle and bone. Voluntary wheel running provides unlimited access without aversive stimuli; but it does not control for duration and intensity. Swimming provides minimal ground reaction forces and aqua therapy is often a physiotherapeutic recommendation for individuals with osteogenesis imperfecta[244], however it may induce physiological stress responses, at least initially, in mice[243]. Unlike previous reports of the positive influence of physical exercise on muscle and bone health[115,212], we found male WT and oim/oim mice exhibited decreases in muscle weight and function and slightly compromised bone microarchitecture and biomechanical properties, while female mice were minimally affected. Sex differences in bone structure and properties in the oim mouse model have previously been reported[245]. One caveat between our exercise study and the previous reports of the positive impact of physical exercises is that our study focused only on aerobic exercise (swimming and voluntary wheel running) while exercise studies in OI patients incorporated a variety of activity including aerobic and strength training exercises[115,212]. In addition, our exercise study focused on mice modeling severe OI while previous reports were in children with mild and moderate OI[115]. This suggests that both the type of exercise and the type of OI are important factors when considering exercise as a potential treatment option. WT mice ran greater than average distances 
compared to their age-matched oim/oim counterparts regardless of sex. This is consistent with previous studies of reduced physical activity levels in oim/oim mice as well as in Collal $^{\mathrm{Jrt} / \mathrm{t}}$ mice, a model of moderately severe OI as a result of a Collal gene defect $[8,163]$. Growing evidence suggests bone is most responsive to dynamic moderate to high magnitude loads, which are quick, short in duration, and non-repetitive in direction[246,247]. Interestingly, WT mice showed positive correlations between total distances run and bone strength, while oim/oim mice did not, which may reflect impaired musculoskeletal response to the weight bearing exercise or that the reduced distances ran, average speeds and time spent on the wheel by the oim/oim mice did not reach the minimal threshold for musculoskeletal responsiveness. The skeleton is striving for the optimum geometry and mass to be able to bear loads while remaining light enough for efficient mobility[126]. Therefore, one should consider that the lack of an overall positive response to the extended running and swimming regimens may also reflect desensitization of the osteocytes to repetitive low magnitude loads[58,248,249]. In separate studies with rats extended durations of running exercise have been shown to not change or to decrease BMD and serum alkaline phosphatase levels, a bone formation biomarker[213,216].

The lack of positive musculoskeletal effects in both WT and oim/oim animals that underwent training regimens suggests that perhaps a more controlled exercise regimen consisting of both endurance and strength training exercises with increasing loads is required to improve muscle and bone properties.

In a previous study, whole muscle homogenates from oim/oim gastrocnemius muscle had increased citrate synthase activity as compared to WT gastrocnemius muscle 
homogenates[163], which led us to hypothesize that oim/oim mice have either 1) increased mitochondrial numbers (mitochondrial mass) or 2) increased mitochondrial function as compared to WT mice. However, our current study demonstrates that oim/oim mice have dramatic reductions in skeletal muscle mitochondrial respiration rates, markers of autophagy and mitophagy, ETC complex IV mitochondrial encoded cytochrome oxidase I, and mitochondrial citrate synthase activity with more mild reductions in mitochondrial DNA /nuclear DNA ratios compared to WT littermates. In addition, markers of mitochondrial biogenesis are increased in oim/oim mice, which may reflect compensatory increases in an attempt to overcome the severely reduced mitochondrial respiration or mitochondrial damage.

The decreases in mitochondrial respiration, ETC complex IV protein content, and mitochondrial DNA copy number may be reflective of the functional oim/oim muscle phenotype of decreased muscle contractile force in oim/oim mice[3]. The mitochondria are responsible for generating the energy necessary for muscle contraction[250,251], and are the primary source of ATP production; ATP is generated by complex V of the ETC chain, ATP synthase, via a proton gradient which is primarily generated and sustained as electrons are transferred through complex IV to oxygen while four protons are simultaneously pumped across the membrane[250,252]. The decreased mitochondrial oxygen consumption in skeletal muscle of oim/oim mice suggests that the oim/oim mitochondrial function is not as efficient as WT mitochondria. Decreased ETC complex IV mitochondrial encoded cytochrome oxidase I in oim/oim gastrocnemius muscle is consistent with the decreased potential to transfer electrons to oxygen which may lead to the inability to maintain an adequate proton gradient. 
Decreased mitochondrial DNA / nuclear DNA ratios suggests decreased mitochondria number[232], although it is controversial as to whether mitochondrial DNA/nuclear DNA ratio is an adequate marker for mitochondrial content especially due to the large variation in DNA copy number per mitochondria[239]. However, increased protein markers of mitochondrial biogenesis, PGC-1 $\alpha$ and TFAM, and decreased LC3-II in isolated mitochondria favor increased mitochondrial number[187,239]. Although, mitochondrial DNA appears reduced in oim/oim gastrocnemius, increased PGC-1 $\alpha$ and TFAM support the hypothesis that oim/oim mice are trying to modulate the signaling pathways in favor of increasing mitochondria, likely to compensate for reduced function. This would be consistent with the indirect measure of mitochondrial content, whole muscle citrate synthase activity, previously observed to be increased in oim/oim whole muscle gastrocnemius protein homogenates[163].

This is the first report of severe muscle weakness in an OI mouse model being attributed, at least in part, to compromised mitochondrial function. Another moderately-severe OI mouse model, Col1a1 ${ }^{\mathrm{Jrt} /+}$, which has an autosomal dominant 18 amino acid deletion in the triple helical domain of the pro 1 (I)collagen chain[51], exhibits reduced physical activity levels with increased oxygen consumption and $\mathrm{CO}_{2}$ production concomitant with increased whole body energy expenditure and altered energy homeostasis[8]. Interestingly, we did not observe any changes in skeletal muscle glycogen content of oim/oim mice compared to WT littermates, further suggesting that the muscle defects, specifically reduced contractile generating force, may be associated with mitochondrial dysfunction, but future studies warrant the inclusion of fatiguing protocols to decipher the role of mitochondria in oim/oim muscle weakness more clearly. Further investigation into 
metabolic homeostasis and energy utilization and expenditure in the oim/oim mouse are critical to understanding the altered mitochondrial function.

Although the role of the extracellular matrix $(\mathrm{ECM})$ as a potential contributing factor to the mitochondrial dysfunction observed in the oim/oim mouse remains to be investigated, the role of the ECM in reduced contractile force and the mechanotransduction of force also needs to be elucidated. The skeletal muscle ECM is hierarchical and can be divided into three structures: the endomysium, perimysium, and epimysium[92,253]. The endomysium is a thin continuous extracellular matrix sheath containing collagen (types III, IV, V, VI, XII, and very little type I collagen) covering the myofibers. The endomysium interacts with the perimysium, which is composed largely of type I collagen and surrounds the muscle fascicles. The perimysial junctional plates (PJP) are the focal site of contact between the perimysium and the myofiber[254]. Through the PJP the perimysium is responsible for the lateral transmission of the contractile forces to the epimysium (largely composed of type I collagen) and the myoteninous junction into the tendon and subsequent tendon-bone interface[ $[92,205,253,254]$. The presence of not only reduced amounts of type I collagen[3], but also abnormal homotrimeric type I collagen in the oim/oim muscle contributes to an altered ECM, which likely disrupts force transmission and altering muscle-bone crosstalk. Passerieux et al. demonstrated intracellularly at the sites of the PJP the presence of type I collagen bundles (perimysial) in association with the subsarcolemmal mitochondria and myonuclei clusters[205]. These findings suggest that although the intermyofibrillar mitochondria of the oim/oim soleus muscle did not appear quantitatively or morphologically abnormal by EM, it is important to evaluate the subsarcolemmal mitochondria. Abnormal muscle ECM has been 
demonstrated by defects in type VI collagen, a component of the perimysium, seen in Bethlem myopathy and Ullrich congenital muscular dystrophy, leading to mitochondrial dysfunction, calcium deregulation, elevated reactive oxygen species, increased apoptosis, and muscle weakness[181,255]. Future investigation into the role of the ECM in oim/oim skeletal muscle weakness may provide insight into the mechanisms of mitochondrial dysfunction in the oim/oim skeletal muscle.

Understanding the mechanisms of muscle weakness in OI are an important consideration for OI treatment, as bone is responsive to the force it feels from muscle contraction. Physiotherapeutic studies that target the muscle weakness in OI are critically needed[256]. Severe muscle weakness or disuse hinder patient quality of life as well as negatively impacting bone health. Understanding the mechanism(s) of muscle weakness in OI will provide new therapeutic targets that will have the potential to improve both muscle and bone strength.

This study is one of the first to demonstrate that extended voluntary wheel running and swimming exercise regimens were well tolerated by the oim/oim mouse a model of moderately severe OI. Although wild-type mice exhibited positive correlations for total distance run and bone biomechanical strength, this was not true for the oim/oim mice, suggesting that the oim/oim mouse muscle-bone unit mechano-responsiveness is compromised. This study is the first to demonstrate reduced mitochondrial function in skeletal muscle as a potential contributing factor to muscle weakness in a mouse model of OI, as evidenced by alterations in mitochondrial content and respiratory function. Further studies including the evaluation of reactive oxygen species and calcium homeostasis are essential to characterize the mechanism of mitochondrial dysfunction in oim/oim mice 
and to understand the impact of the mitochondrial energy utilization and expenditure on muscle weakness. Exploring the mechanisms responsible for muscle weakness and whole-body metabolic alterations promises to provide important insight into the musculoskeletal pathogenesis of OI.

Authors' roles: Study design: VLG, YJ, CLP, RSR. Study conduct: muscle (YJ), mouse exercise (YJ), bone biomechanics (YJ), mitochondrial markers in whole muscle homogenate (VLG, RPC, and GMM), mitochondrial isolation experiments (VLG and GMM), and electron microscopy (VLG). Data analysis: VLG, YJ, RPC, GMM, RSR, and CLP. Drafting manuscript: VLG, YJ, RPC, GMM, RSR, and CLP. Revising manuscript content: VLG, YJ, RPC, GMM, RSR, and CLP. Approving final version of the manuscript: VLG, YJ, RPC, GMM, RSR, and CLP. CLP takes responsibility for the integrity of the data analysis. 


\section{Supplementary Material for Chapter II}

Table II-S1. The swimming exercise regimen of wild-type and oim/oim mice was initiated at 6 weeks of age and completed at 4 months of age.

\begin{tabular}{|c|c|c|c|c|c|c|}
\hline Week 1 & $\begin{array}{l}\text { Swim } \\
\text { (min) }\end{array}$ & $\begin{array}{l}\text { Rest } \\
\text { (min) }\end{array}$ & $\begin{array}{l}\text { Swim } \\
\text { (min) }\end{array}$ & $\begin{array}{c}\text { Rest } \\
\text { (min) }\end{array}$ & $\begin{array}{l}\text { Swim } \\
\text { (min) }\end{array}$ & $\begin{array}{l}\text { Total Swim } \\
\text { Time (min) }\end{array}$ \\
\hline Day 1 & 2 & N/A & N/A & N/A & N/A & 2 \\
\hline Day 2 & 5 & N/A & N/A & N/A & N/A & 5 \\
\hline Day 3 & 8 & N/A & N/A & N/A & N/A & 8 \\
\hline Day 4 & 6 & 3 & 6 & N/A & N/A & 12 \\
\hline Day 5 & 8 & 3 & 8 & N/A & N/A & 16 \\
\hline \multicolumn{7}{|l|}{ Week 2} \\
\hline Day 6 & 10 & 3 & 10 & N/A & N/A & 20 \\
\hline Day 7 & 12 & 3 & 12 & N/A & N/A & 24 \\
\hline Day 8 & 14 & 3 & 14 & N/A & N/A & 28 \\
\hline Day 9 & 10 & 3 & 12 & 3 & 10 & 32 \\
\hline Day 10 & 12 & 3 & 12 & 3 & 12 & 36 \\
\hline \multicolumn{7}{|l|}{ Week 3} \\
\hline Day 11 & 14 & 3 & 12 & 3 & 14 & 40 \\
\hline Day 12 & 16 & 3 & 14 & 3 & 16 & 44 \\
\hline Day 13 & 16 & 3 & 16 & 3 & 16 & 48 \\
\hline Day 14 & 18 & 3 & 16 & 3 & 18 & 52 \\
\hline Day 15 & 19 & 3 & 18 & 3 & 19 & 56 \\
\hline \multicolumn{7}{|l|}{ Week 4} \\
\hline $\begin{array}{l}\text { Days 16- } \\
20\end{array}$ & 20 & 3 & 20 & 3 & 20 & 60 \\
\hline \multicolumn{7}{|l|}{ Week 5} \\
\hline $\begin{array}{l}\text { Days 21- } \\
25\end{array}$ & 20 & 3 & 20 & 3 & 20 & 60 \\
\hline \multicolumn{7}{|l|}{ Week 6} \\
\hline $\begin{array}{l}\text { Days } 26- \\
30\end{array}$ & 20 & 3 & 20 & 3 & 20 & 60 \\
\hline \multicolumn{7}{|l|}{ Week 7} \\
\hline $\begin{array}{l}\text { Days 31- } \\
35\end{array}$ & 20 & 3 & 20 & 3 & 20 & 60 \\
\hline \multicolumn{7}{|l|}{ Week 8} \\
\hline $\begin{array}{l}\text { Days 36- } \\
40\end{array}$ & 20 & 3 & 20 & 3 & 20 & 60 \\
\hline \multicolumn{7}{|l|}{ Week 9} \\
\hline $\begin{array}{l}\text { Days 41- } \\
45\end{array}$ & 20 & 3 & 20 & 3 & 20 & 60 \\
\hline \multicolumn{7}{|l|}{ Week 10} \\
\hline $\begin{array}{l}\text { Days 46- } \\
50\end{array}$ & 20 & 3 & 20 & 3 & 20 & 60 \\
\hline
\end{tabular}


Table II-S2. Femoral microarchitecture and biomechanical properties of male WT and oim/oim in-cage control, volunteer wheel running, and swimming exercised mice.

\begin{tabular}{|c|c|c|c|c|c|c|}
\hline & \multicolumn{3}{|c|}{ WT } & \multicolumn{3}{|c|}{ oim/oim } \\
\hline & $\begin{array}{c}\text { Control } \\
(\mathrm{n}=9-11)\end{array}$ & $\begin{array}{l}\text { Wheel } \\
(\mathrm{n}=11)\end{array}$ & $\begin{array}{c}\text { Swim } \\
(\mathrm{n}=8-10)\end{array}$ & $\begin{array}{l}\text { Control } \\
(\mathrm{n}=8-9)\end{array}$ & $\begin{array}{l}\text { Wheel } \\
(\mathrm{n}=9)\end{array}$ & $\begin{array}{c}\text { Swim } \\
(\mathrm{n}=8-9)\end{array}$ \\
\hline \multicolumn{7}{|c|}{ Cortical Bone Microarchitecture $^{\text {a }}$} \\
\hline FL & $16.07 \pm 0.26$ & $15.46 \pm 0.29^{\dagger}$ & $15.86 \pm 0.24$ & $16.09 \pm 0.34$ & $15.47 \pm 0.34^{* \dagger}$ & $15.89 \pm 0.29$ \\
\hline $\begin{array}{l}\mathrm{MC} \\
\mathrm{D}\end{array}$ & $1.00 \pm 0.09$ & $0.90 \pm 0.05^{\dagger}$ & $1.09 \pm 0.08^{\dagger}$ & $0.96 \pm 0.04$ & $0.96 \pm 0.07$ & $0.96 \pm 0.07$ \\
\hline $\begin{array}{l}\mathrm{CB} \\
\mathrm{W}\end{array}$ & $0.40 \pm 0.04$ & $0.35 \pm 0.02^{\dagger}$ & $0.34 \pm 0.02^{\dagger}$ & $0.33 \pm 0.01^{*}$ & $0.30 \pm 0.03^{*}$ & $0.32 \pm 0.03^{*}$ \\
\hline K & $0.86 \pm 0.16$ & $0.53 \pm 0.03^{\dagger}$ & $0.72 \pm 0.09^{\dagger}$ & $0.52 \pm 0.08^{*}$ & $0.43 \pm 0.06^{*}$ & $0.50 \pm 0.07^{*}$ \\
\hline \multicolumn{7}{|c|}{ Torsional Loading to Failure ${ }^{b}$} \\
\hline $\mathrm{T}_{\max }$ & $43.26 \pm 6.49$ & $29.37 \pm 3.61^{\dagger}$ & $38.36 \pm 3.96$ & $9.76 \pm 6.38^{*}$ & $9.27 \pm 4.00^{*}$ & $9.61 \pm 2.71^{*}$ \\
\hline Ks & $55.86 \pm 11.12$ & $47.74 \pm 9.21^{\dagger}$ & $59.34 \pm 14.31$ & $28.68 \pm 10.24^{*}$ & $29.18 \pm 6.74^{*}$ & $31.86 \pm 8.40^{*}$ \\
\hline $\mathrm{Su}$ & $52.10 \pm 7.20$ & $50.06 \pm 6.62$ & $51.14 \pm 5.06$ & $17.90 \pm 13.07^{*}$ & $18.15 \pm 7.54^{*}$ & $16.98 \pm 5.09^{*}$ \\
\hline G & $\begin{array}{l}489.16 \pm 130 . \\
67\end{array}$ & $\begin{array}{l}658.12 \pm 188.3 \\
7^{\dagger}\end{array}$ & $\begin{array}{l}558.72 \pm 117 . \\
37\end{array}$ & $\begin{array}{l}417.24 \pm 189 \\
57\end{array}$ & $\begin{array}{l}475.37 \pm 105 \\
48\end{array}$ & $\begin{array}{l}453.21 \pm 136 \\
85\end{array}$ \\
\hline $\mathrm{U}$ & $9.75 \pm 2.55$ & $6.45 \pm 1.25^{\dagger}$ & $8.53 \pm 1.16$ & $1.67 \pm 1.59^{*}$ & $1.54 \pm 1.12^{*}$ & $1.57 \pm 0.73^{*}$ \\
\hline
\end{tabular}

${ }^{a}$ Femur Length (FL); Marrow Cavity Diameter (MCD); Cortical Bone Width (CBW); Polar moment of area (K)

${ }^{\mathrm{b}}$ Torsional ultimate force $\left(\mathrm{T}_{\max }\right)$; Torsional stiffness $(\mathrm{Ks})$; Tensile strength $(\mathrm{Su})$; Shear modulus of elasticity $(\mathrm{G})$; Energy to failure (U)

Values are presented as MEAN \pm STDEV. ${ }^{*} p \leq 0.05$ vs. WT; ${ }^{\dagger} p \leq 0.05$ vs. in-cage control 
Table II-S3. Femoral microarchitecture and biomechanical properties of female WT and oim/oim in-cage control, volunteer wheel running, and swimming exercised mice.

\begin{tabular}{|c|c|c|c|c|c|c|}
\hline & \multicolumn{3}{|c|}{ WT } & \multicolumn{3}{|c|}{ oim/oim } \\
\hline & $\begin{array}{c}\text { Control } \\
(\mathrm{n}=8)\end{array}$ & $\begin{array}{c}\text { Wheel } \\
(\mathrm{n}=9-11)\end{array}$ & $\begin{array}{c}\text { Swim } \\
(\mathrm{n}=11-12)\end{array}$ & $\begin{array}{l}\text { Control } \\
(\mathrm{n}=7-9)\end{array}$ & $\begin{array}{c}\text { Wheel } \\
(\mathrm{n}=8-12)\end{array}$ & $\begin{array}{c}\text { Swim } \\
(\mathrm{n}=4-13)\end{array}$ \\
\hline \multicolumn{7}{|c|}{ Cortical Bone Microarchitecture ${ }^{\text {a }}$} \\
\hline FL & $15.77 \pm 0.37$ & $15.71 \pm 0.22$ & $15.58 \pm 0.44$ & $15.44 \pm 0.49$ & $15.29 \pm 0.45^{*}$ & $15.39 \pm 0.30^{*}$ \\
\hline $\begin{array}{l}\mathrm{MC} \\
\mathrm{D}\end{array}$ & $0.90 \pm 0.05$ & $0.89 \pm 0.05$ & $0.92 \pm 0.05$ & $0.92 \pm 0.06$ & $0.86 \pm 0.08^{\dagger}$ & $0.91 \pm 0.06$ \\
\hline $\begin{array}{l}\text { CB } \\
W\end{array}$ & $0.36 \pm 0.02$ & $0.35 \pm 0.02$ & $0.34 \pm 0.02$ & $0.29 \pm 0.03^{*}$ & $0.33 \pm 0.04^{\dagger}$ & $0.29 \pm 0.02^{*}$ \\
\hline K & $0.53 \pm 0.06$ & $0.50 \pm 0.05$ & $0.53 \pm 0.09$ & $0.37 \pm 0.05^{*}$ & $0.41 \pm 0.08^{*}$ & $0.38 \pm 0.08^{*}$ \\
\hline \multicolumn{7}{|c|}{ Torsional Loading to Failure ${ }^{b}$} \\
\hline $\mathrm{T}_{\max }$ & $33.14 \pm 3.49$ & $31.38 \pm 1.46$ & $26.71 \pm 3.56^{\dagger}$ & $13.54 \pm 1.43^{*}$ & $11.07 \pm 4.88^{*}$ & $11.00 \pm 3.13^{* \dagger}$ \\
\hline Ks & $45.38 \pm 10.06$ & $42.20 \pm 2.88$ & $47.07 \pm 15.28$ & $36.29 \pm 8.22^{*}$ & $33.90 \pm 8.68^{*}$ & $30.51 \pm 9.10^{*}$ \\
\hline $\mathrm{Su}$ & $55.37 \pm 3.13$ & $54.71 \pm 3.82$ & $44.21 \pm 6.94^{\dagger}$ & $29.20 \pm 3.27^{*}$ & $23.06 \pm 9.62^{*}$ & $24.22 \pm 6.30^{*}$ \\
\hline G & $\begin{array}{l}598.80 \pm 127.9 \\
4\end{array}$ & $\begin{array}{l}593.44 \pm 67.8 \\
5\end{array}$ & $\begin{array}{l}654.22 \pm 288.8 \\
7\end{array}$ & $\begin{array}{l}682.95 \pm 196.2 \\
6\end{array}$ & $\begin{array}{l}605.64 \pm 169.0 \\
3\end{array}$ & $\begin{array}{l}596.25 \pm 202.2 \\
7\end{array}$ \\
\hline $\mathrm{U}$ & $7.58 \pm 1.09$ & $7.48 \pm 0.91$ & $6.50 \pm 1.80$ & $2.44 \pm 0.71^{*}$ & $1.86 \pm 1.19^{*}$ & $1.95 \pm 0.87^{*}$ \\
\hline
\end{tabular}

${ }^{a}$ Femur Length (FL); Marrow Cavity Diameter (MCD); Cortical Bone Width (CBW); Polar moment of area $(\mathrm{K})$

${ }^{\mathrm{b}}$ Torsional ultimate force $\left(\mathrm{T}_{\max }\right)$; Torsional stiffness $(\mathrm{Ks})$; Tensile strength $(\mathrm{Su})$; Shear modulus of elasticity $(\mathrm{G})$; Energy to failure (U)

Values are presented as MEAN \pm STDEV. ${ }^{*} p \leq 0.05$ vs. WT; ${ }^{\dagger} p \leq 0.05$ vs. in-cage control 

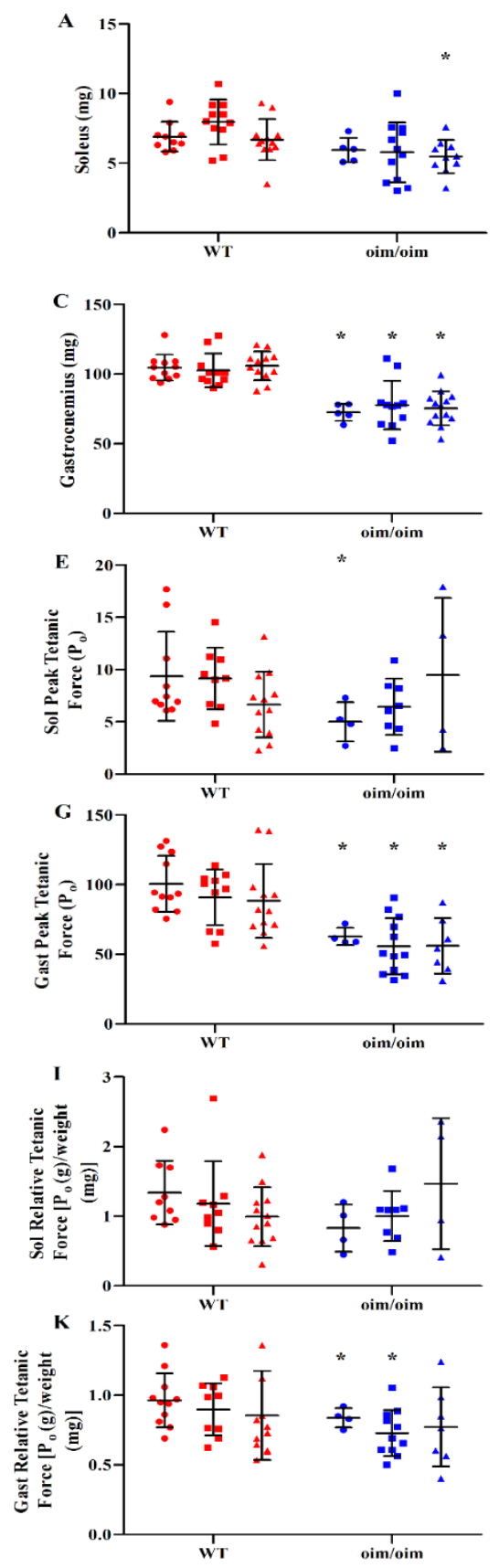
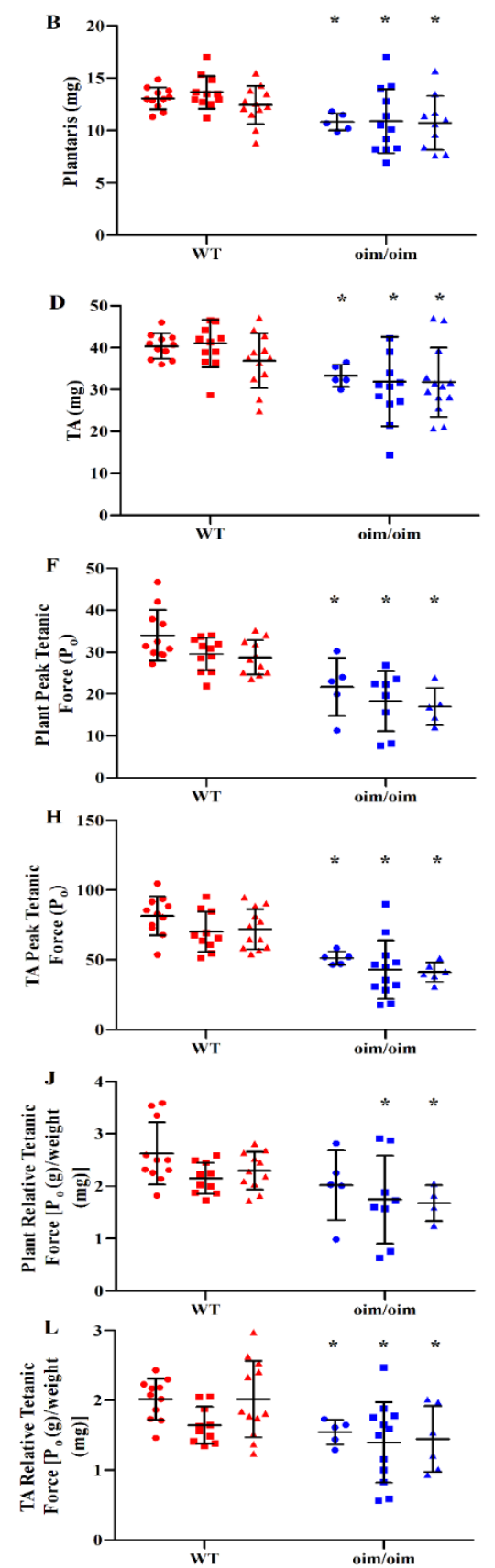
Figure II-S1: Volunteer wheel running and swimming exercised female oim/oim mice had reduced hindlimb skeletal muscle weight and peak tetanic force as compared to incage control counterparts. (A) Soleus , (B), Plantaris, (C) Gastrocnemius, (D) Tibialis Anterior muscle wet weights, (E) Soleus peak tetanic force $\left(\mathrm{P}_{\mathrm{o}}\right)$, (F) Plantaris Peak $\mathrm{P}_{\mathrm{o}}$, (G) Gastrocnemius Peak $P_{o}$, (H) Tibialis Anterior Peak $P_{o}$, (I) Soleus relative $P_{o}$, (J) Plantaris relative $P_{0},(K)$ Gastrocnemius relative $P_{o}$, (L) Tibialis Anterior relative $P_{o}$ of 4 months old female WT and oim/oim in-cage control, volunteer wheel running, and swimming exercised mice. Graphs are presented as MEAN \pm STDEV. ${ }^{*} \mathrm{p} \leq 0.05$ vs. WT; ( $\mathrm{n}=4-13$ per groups). Red and blue symbols represent WT and oim/oim, respectively. Circles, squares, and triangles represent control, wheel running, and swimming, respectively. 
A

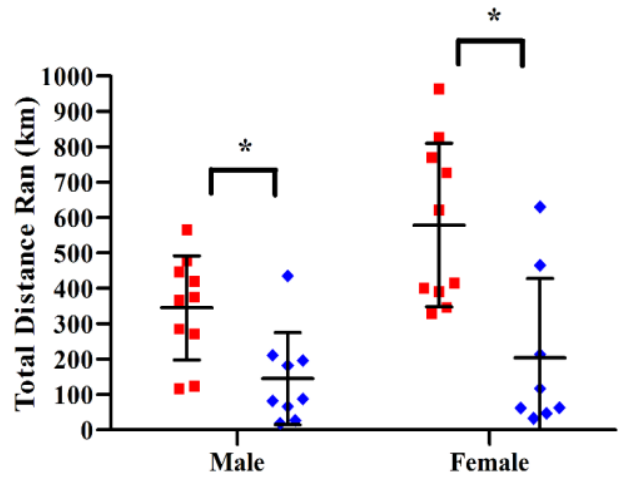

C

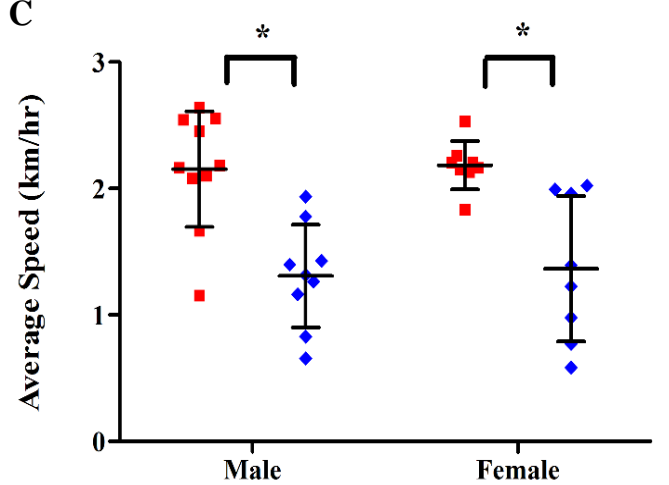

E

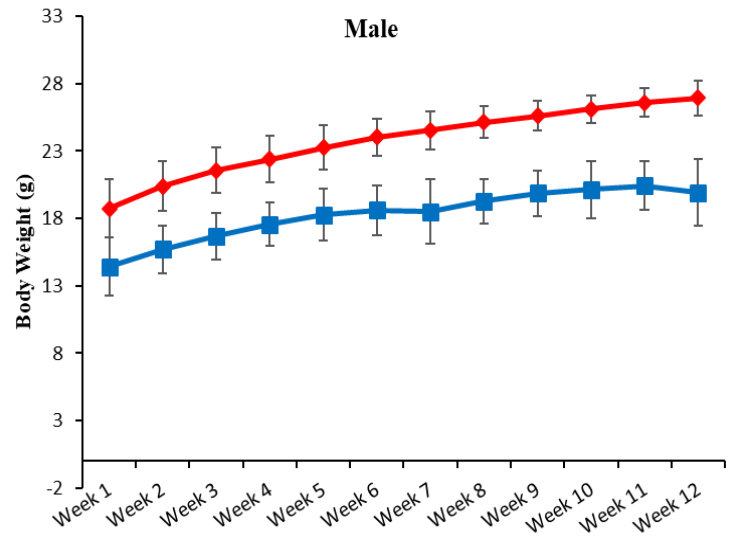

B

D
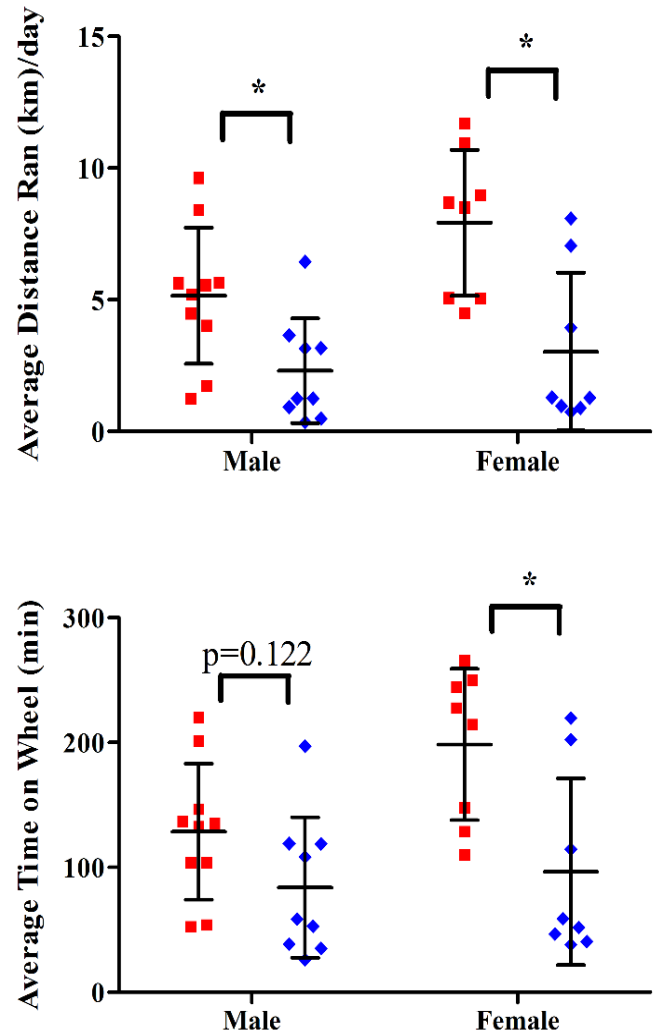

F

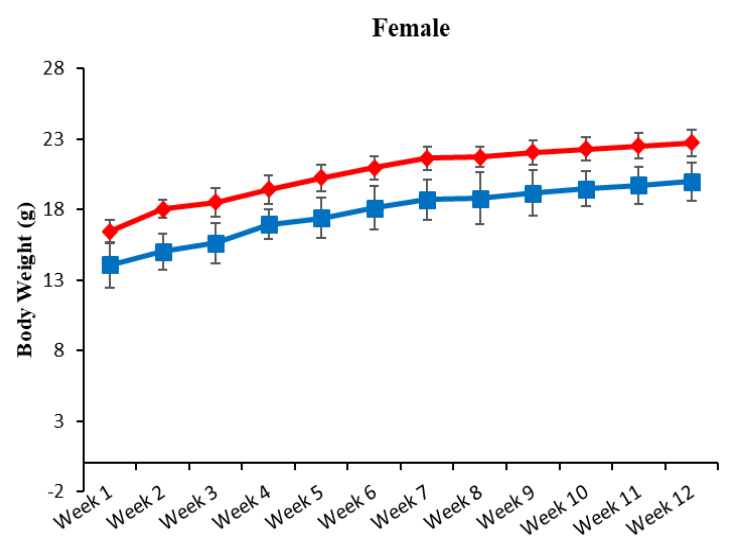


$\mathbf{G}$

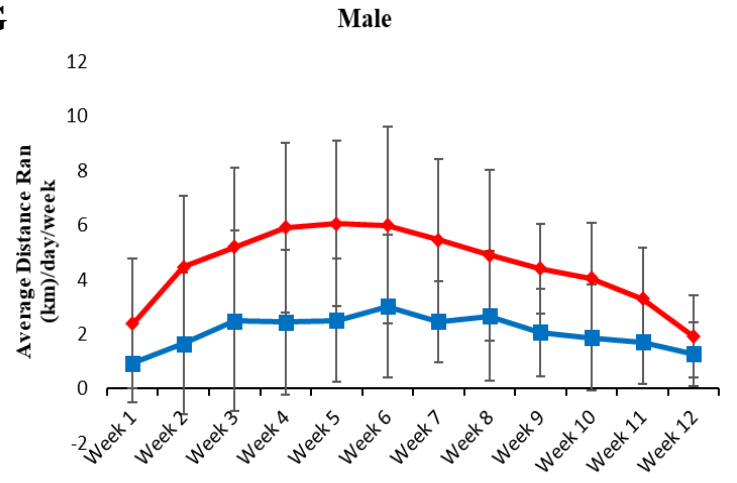

I

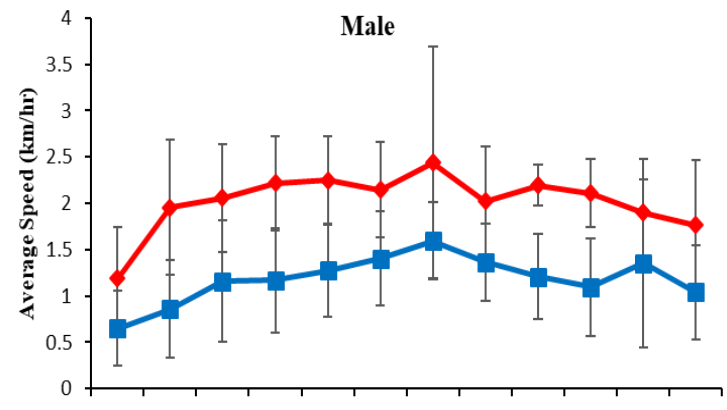

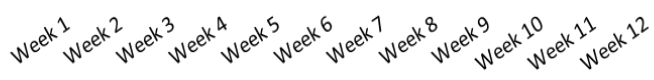

$\mathbf{K}$

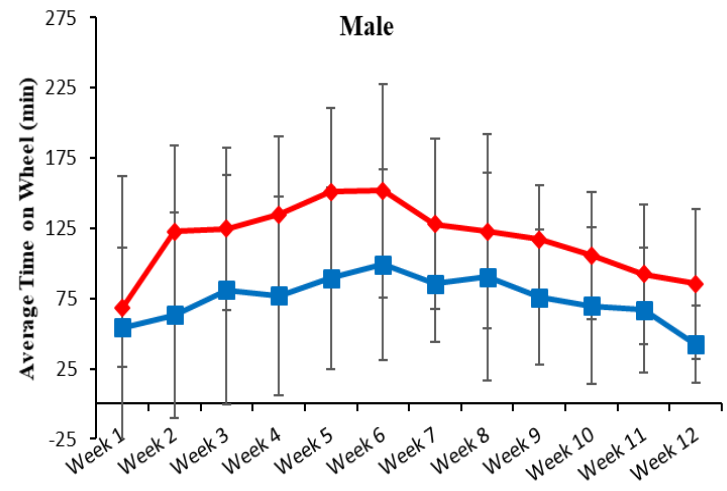

$\mathbf{H}$

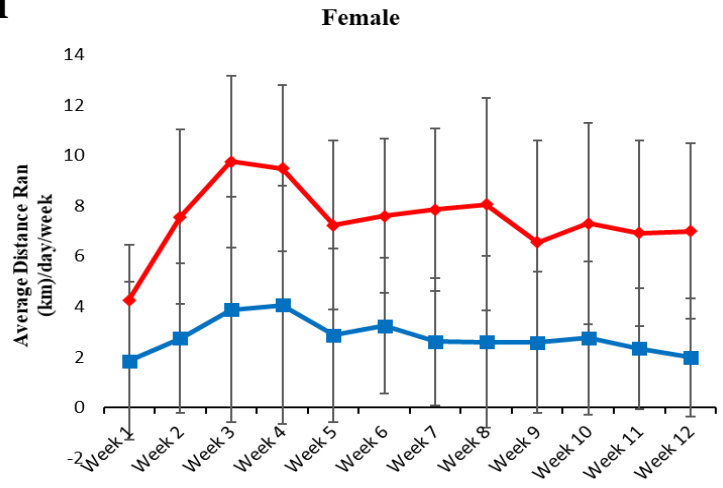

$\mathbf{J}$

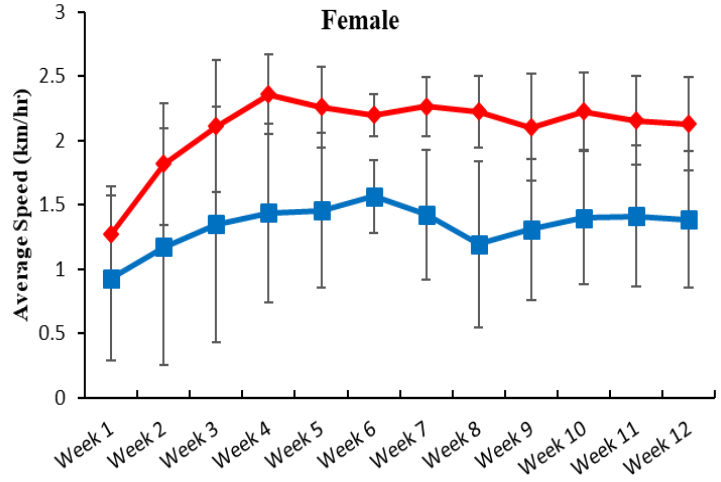

$\mathbf{L}$

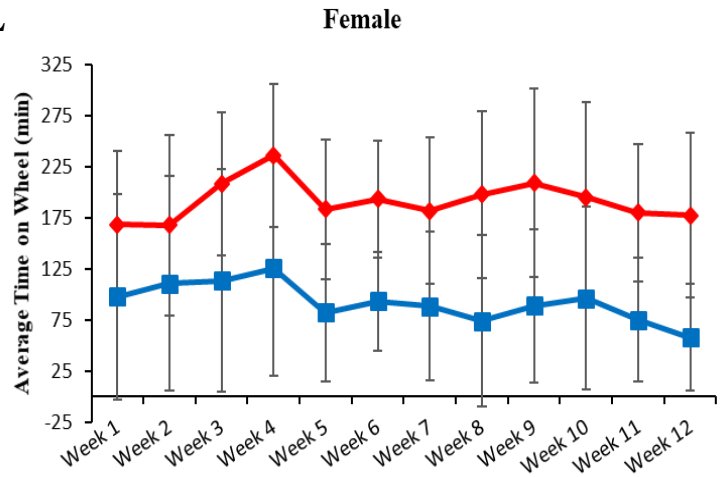

Figure II-S2: (A) Average of the total distance $(\mathrm{km})$ ran by male and female oim/oim (blue) mice was significantly less than wild-type ( $\mathrm{red}$ ) mice. Average of weeks 2-10 for (B) average distance ran $(\mathrm{km})$ per day, (C) average speed $(\mathrm{km} / \mathrm{hr})$, and (D) average time spent on the wheel (mins) were reduced for oim/oim (blue) mice compared to wild-type (red) mice. Average body weight (g) per week of (E) male and (F) female WT and oim/oim mice. Average distance ran per day per week of $(\mathrm{G})$ male and $(\mathrm{H})$ female WT and oim/oim mice. Average speed $(\mathrm{km} / \mathrm{hr}$ ) ran per week of (I) male and (J) female WT and oim/oim mice. Average time on the wheel (minutes) per week of (K) male and (L) female WT and oim/oim mice. Graphs are presented as MEAN \pm STDEV. ${ }^{*} \mathrm{p} \leq 0.05$ vs. WT. ( $=9-12$ per group) 


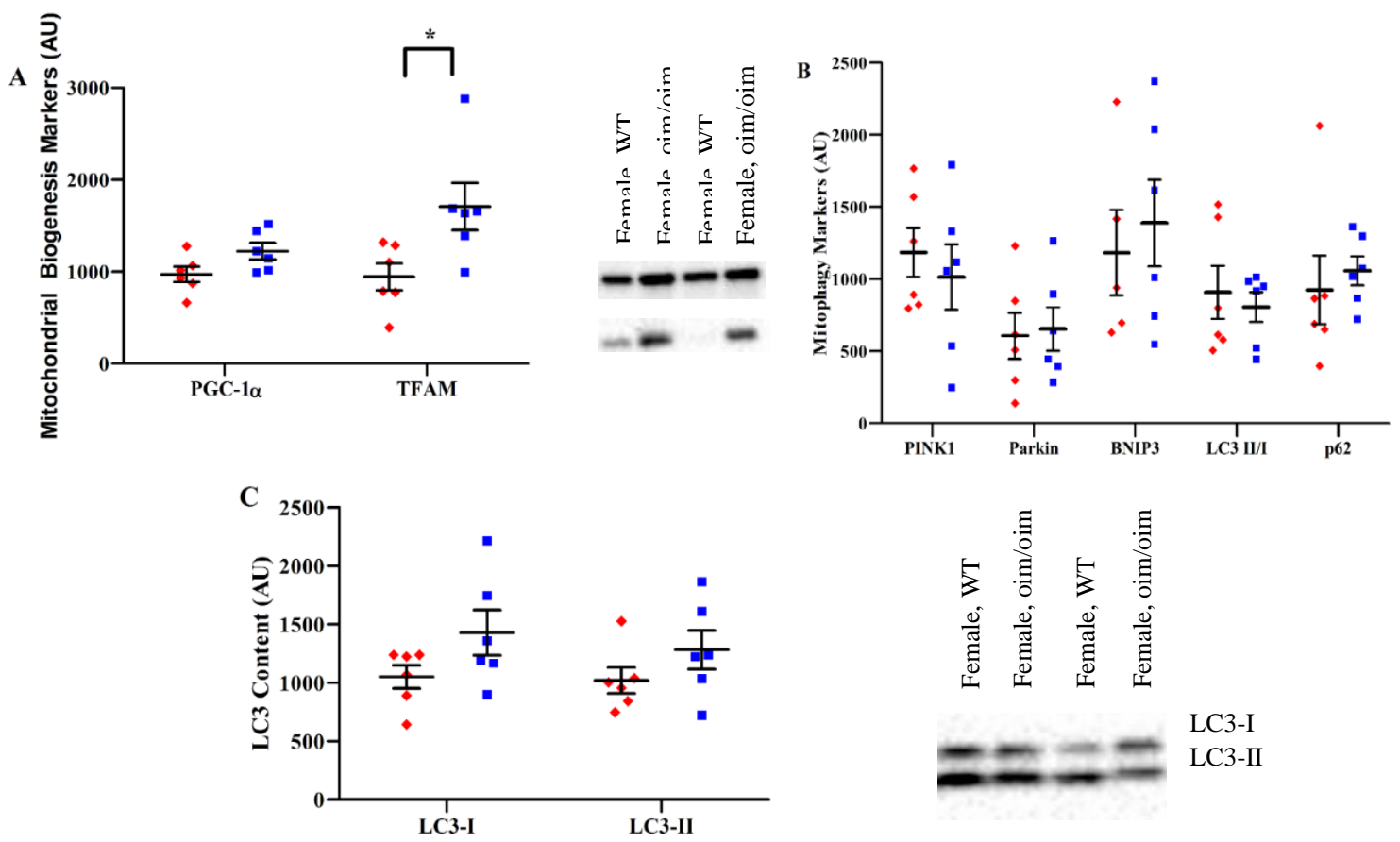

Figure II-S3: (A) Female 4-month-old oim/oim mixed gastrocnemius muscle homogenates exhibit increased TFAM, a marker of mitochondrial biogenesis. Western blot analysis of markers of (B) autophagy and mitophagy in protein isolated from 4month-old female oim/oim (blue symbols) mixed gastrocnemius muscle did not change compared to WT (red symbols). (C) LC3-I and LC3-II were quantified separately. Graphs are presented as MEANS \pm SE. ${ }^{*} \mathrm{p} \leq 0.05$ vs. WT. ( $\mathrm{n}=5-6$ per group). 


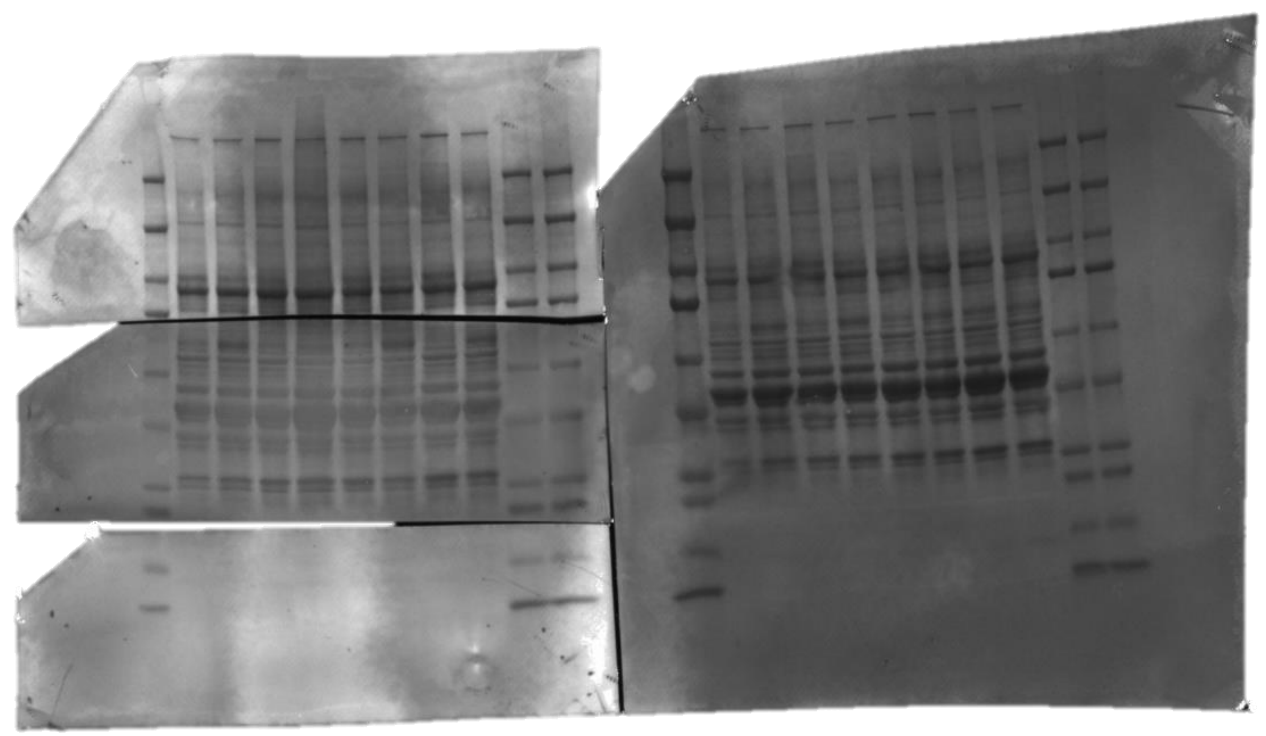

Figure II-S4: A representative blots (for western blot analyses) following transfer and staining with the amido black stain for total protein content to show transfer quality and for normalization of the western blot quantification (Figures II-3, 5 \& 6). 


\section{CHAPTER III}

SKELETAL MUSCLE SPECIFIC MITOCHONDRIAL DYSFUNCTION AND ALTERED ENERGY METABOLISM IN A MURINE MODEL (OIM/OIM) OF SEVERE OSTEOGENESIS IMPERFECTA

This chapter has been published in the Journal of Molecular Genetics and Metabolism

Gremminger, V.L.; Harrelson, E.N.; Crawford, T.K.; Ohler, A.; Schulz, L.C.; Rector, R.S.; Phillips, C.L. Skeletal muscle specific mitochondrial dysfunction and altered energy metabolism in a murine model (oim/oim) of severe osteogenesis imperfecta. Mol. Genet. Metab. 2021, doi:10.1016/j.ymgme.2021.02.004.

Victoria L. Gremminger ${ }^{1}$, Emily N. Harrelson ${ }^{1}$, Tara K Crawford ${ }^{1}$, Adrienne Ohler ${ }^{2}$, Laura C. Schulz ${ }^{3}$, R. Scott Rector ${ }^{4}$, Charlotte L. Phillips ${ }^{1,2}$

${ }^{1}$ Department of Biochemistry, University of Missouri, Columbia, MO 65211

2 Department of Child Health, University of Missouri, Columbia, MO 65211

${ }^{3}$ Department of Obstetrics, Gynecology, and Women's Health; University of Missouri, Columbia, MO 65211

${ }^{4}$ Departments of Nutrition and Exercise Physiology and Medicine-GI, University of Missouri; Harry S Truman Memorial VA Hospital, Columbia, MO 65211 


\begin{abstract}
Osteogenesis imperfecta (OI) is a heritable connective tissue disorder with patients exhibiting bone fragility and muscle weakness. The synergistic biochemical and biomechanical relationship between bone and muscle is a critical potential therapeutic target, such that muscle weakness should not be ignored. Previous studies demonstrated mitochondrial dysfunction in the skeletal muscle of oim/oim mice, which model a severe human type III OI. Here, we further characterize this mitochondrial dysfunction and evaluate several parameters of whole body and skeletal muscle metabolism. We demonstrate reduced mitochondrial respiration in female gastrocnemius muscle, but not in liver or heart mitochondria, suggesting that mitochondrial dysfunction is not global in the oim/oim mouse. Myosin heavy chain fiber type distributions were altered in the oim/oim soleus muscle with a decrease (-33 to 50\%) in type I myofibers and an increase $(+31 \%)$ in type IIa myofibers relative to their wildtype (WT) littermates. Additionally, altered body composition and increased energy expenditure were observed oim/oim mice relative to WT littermates. These results suggest that skeletal muscle mitochondrial dysfunction is linked to whole body metabolic alterations and to skeletal muscle weakness in the oim/oim mouse.
\end{abstract}




\section{Introduction}

Osteogenesis imperfecta (OI), also referred to as brittle bone disease, is a rare, heritable connective tissue disorder affecting approximately 1 in 15,000 births $[1,17]$. Roughly $85 \%$ of OI cases arise due to mutations in the type I collagen genes, COL1A1 and COL1A2, while the remaining $15 \%$ result from mutations in genes involved in posttranslational modification of type I collagen, osteoblast maturation or mineralization [1]. Type I collagen, the most abundant of the collagens, is an important structural protein composing the majority of the organic component of bone. OI is an extremely heterogeneous disease in terms of severity with the most common and striking feature being bone fragility. There are four classical Sillence types of OI: type I is the least severe, type II results in perinatal lethality, type III being the most severe viable form, and type IV results in a moderate phenotype [13]. In addition to bone fragility, clinical manifestations include short stature and craniofacial abnormalities, with more recent investigations also highlighting the presence of an inherent muscle pathology in both mouse models and patients with OI $[3,4,6,48]$. However, current treatment strategies for patients with OI focus primarily on bone fragility, with emphasis on preventing bone resorption (bisphosphonates) and/or surgical rodding interventions[17]. Understanding the mechanisms of muscle weakness in OI could potentiate novel therapies to improve muscle function and ultimately bone strength.

Muscle weakness has been implicated as an inherent part of the pathophysiology of OI through investigation of the osteogenesis imperfecta murine (oim) model[3]. The oim mouse Colla2 gene contains a nucleotide deletion, leading to a frameshift in the carboxyterminal end resulting in a nonfunctional proa2(I) collagen. This ultimately leads to 
production of homotrimeric type I collagen, $\left[\alpha 1(\mathrm{I})_{3]}\right.$, rather than the normal heterotrimeric type I collagen, $\left[\alpha 1(\mathrm{I})_{2} \alpha 2(\mathrm{I})\right][39]$. The homozygous (oim/oim) mice model severe human type III OI, exhibiting severe bone fragility, decreased bone mineral density, and small body size compared to wildtype (WT) littermates[39]. Oim/oim mice have significantly reduced muscle size as well as reduced specific contractile generating force [contractile force normalized to muscle cross sectional area][3]. Subsequently, numerous reports have revealed muscle weakness in OI patients, including that $80 \%$ of patients with mild/moderate OI experience muscle force deficits $[4,6,47,48]$. Because muscle and bone are highly synergistic tissues which "talk" to one another through both chemical signals and physical forces, it is important to understand this muscle weakness in OI in order to exploit this relationship to ultimately improve bone strength through the improvement of muscle function $[14,57,257]$. We recently demonstrated that oim/oim hindlimb skeletal muscle exhibits severe mitochondrial dysfunction and altered mitochondrial numbers, which likely contributes to their muscle weakness [162][258].

Evidence of metabolic alterations in OI, though limited, have been observed in both mouse models and the patient population. Previous evidence found that prepubescent OI patients exhibited higher body temperatures and a $45 \%$ increase in metabolic rate, but did not show altered respiratory quotients relative to children without OI[7]. Interestingly, they also reported that these changes subsided in patients after puberty, as do the overall fracture rates suggesting a link between hyper-metabolism, fractures, and sexhormones[7]. More recently, evidence of metabolic alterations were demonstrated in a mouse model of severe OI; 4 week old Collal ${ }^{J t t /+}$ mice (modeling human OI type IV/III) exhibited sex-dependent changes in metabolism that normalized by puberty ( 8 weeks of 
age) [8]. These alterations included increased insulin levels and respiratory exchange rate, in males, as well as improved glucose tolerance in females Additionally, a previous report found evidence that a patient with OI type III/IV, initially suspected to have malignant hyperthermia, was in a hypermetabolic state which was likely related to a primary type I collagen defect [259].

Based on these previous reports of metabolic alterations in both patients and the Colla $1^{\mathrm{Irt+}+}$ mouse as well as our recent findings of mitochondrial dysfunction, we sought to investigate the potential of metabolic dysfunction in the oim/oim mouse. The current study provides further investigations of mitochondrial function and metabolism in the oim/oim mouse, by 1) addressing whether the skeletal muscle mitochondrial dysfunction is more global or tissue specific by evaluating cardiac muscle and liver mitochondrial function, 2) evaluating skeletal muscle fiber type distributions, and 3) evaluating several metabolic parameters including measures of whole body energy expenditure and skeletal muscle fatty acid oxidation relative to wildtype littermates. Better characterization and understanding of these alterations provide insight into the skeletal muscle weakness that occurs in OI and the nature of the relationship between mitochondrial function and type I collagen mutations and promise to pave the way for new therapies that will improve muscle function and ultimately bone strength. 


\section{Methods}

\section{Mice}

Col1a $2^{\text {oim }}$ mice (stock \#001815 (oim)) were originally obtained from Jackson Laboratory (Bar Harbor, ME, USA). All mice were maintained on the C57BL/6 J background and genotyped as previously described $[39,224]$. Non-breeding male and female wild type (WT) and oim/oim adult mice (13-19 weeks of age) were used for all experiments described, except for an additional subset of 8 week old WT and oim/oim males whose gastrocnemius and quadriceps muscles were used to assess mitochondrial respiration. Age- and sex- matched WT littermates were used as control animals. All mice were given ad libitum access to food (stock chow, Purina Lab Diet 5053) and water. Mice were housed in an AAALAC-accredited facility at the University of Missouri; all experimental manipulations were performed under an approved University of Missouri Animal Care and Use Committee protocol. At the time of sacrifice, a mixture of xylazine/ketamine anesthesia was used followed by cardiac puncture (serum collected for future experiments), and cervical dislocation.

\section{Mitochondria Isolation, Respiration, and $\mathrm{H}_{2} \mathrm{O}_{2}$ Analyses}

Mitochondria were isolated from heart, liver, and mixed skeletal muscles including the gastrocnemius and quadriceps muscles (each muscle contains both white/fast-twitch/type II and red/slow-twitch/type I myofibers) following a series of homogenization and differential centrifugation procedures as previously described $[227,229,260]$. Mitochondria isolated from heart, liver and gastrocnemius and quadriceps muscles were used to assess mitochondrial respiration via high-resolution respirometry, while 
mitochondria isolated from gastrocnemius muscle were also used to determine mitochondrial $\mathrm{H}_{2} \mathrm{O}_{2}$ production.

High-resolution respirometry was used to measure mitochondrial respiration by the isolated liver, heart, gastrocnemius (gast), and quadriceps (quad) muscle mitochondria using the Oroboros Oxygraph-2k (Oroboros Instruments, Innsbruck, Austria) following a modified protocol[227,258,261]. $20 \mu \mathrm{L}$ of isolated mitochondria were added to the respiration chambers to measure basal respiration. Steady-state oxygen flux was measured by the addition of $2 \mathrm{mM}$ malate and $5 \mathrm{mM}$ glutamate. Oxygen flux through complex I was measured by the titration of ADP [50 to $125 \mu \mathrm{M}$ (liver), $25-125 \mu \mathrm{M}$ (heart), or 125-375 $\mu \mathrm{M}$ (gast and quad)]; through complex I + II by the titration of succinate [5 to $7.5 \mathrm{mM}$ (liver), $10 \mathrm{mM}$ (heart), or 1-5 $\mathrm{mM}$ (gast and quad)], and maximal uncoupled respiration was measured by the titration of carbonyl cyanide-4(trifluoromethoxy) phenylhydrazone (FCCP) [0.125-0.25 $\mu \mathrm{M}$ (liver), $0.25 \mu \mathrm{M}$ (heart), or $0.125 \mu \mathrm{M}$ (gast and quad)]. Finally, cytochrome $\mathrm{c}$ was added [5 $\mu \mathrm{M}$ (liver and heart) and $10 \mu \mathrm{M}$ (gast and quad)] to assess the quality of mitochondrial isolation. All measurements were normalized to the mitochondrial protein content.

An Oroboros Oxygraph-2k (Oroboros Instruments, Innsbruck, Austria) equipped with a fluorimeter was used to measure $\mathrm{H}_{2} \mathrm{O}_{2}$ production in mitochondria isolated from gastrocnemius muscles. Amplex Red (ThermoFisher Scientific; Waltham, MA) and horseradish peroxidase (HRP) were added to the respiration chamber followed by the addition of $20 \mathrm{uL}$ of isolated mitochondria. $20-70 \mathrm{uM}$ of palmitoyl-CoA was titrated into the respiration chambers and the amount of fluorescence assessed. 


\section{Citrate Synthase Activity}

Citrate synthase activity was measured in gastrocnemius, liver, and heart mitochondria as previously described[233,258].

\section{Skeletal Muscle Mitochondrial Fatty Acid Oxidation}

Complete fatty acid oxidation leads to the production of carbon dioxide while incomplete fatty acid oxidation results in the generation of acid soluble metabolite (ASMs). Complete, incomplete, and total [Incomplete (ASM) + Complete $\left.\left(\mathrm{CO}_{2}\right)\right]$ oxidation of (1-

${ }^{14} \mathrm{C}$ ) palmitate were measured in mitochondria isolated from gastrocnemius muscle as well as in whole quadriceps muscle homogenate as previously described [262].

\section{Myosin Heavy Chain Immunohistochemical Analysis}

Skeletal muscles can be characterized according to their oxidative capacity as type I slow twitch fibers and type II fast twitch fibers, also known as red and white muscle, respectively [79]. Furthermore, type II fibers can be further classified as type IIa, type IIb, and type IIx representing a fast oxidative, fast glycolytic and combination, respectively. Due to their increased endurance and oxidative properties, type I fibers tend to have more mitochondria relative to other fiber types[78]. Using myosin heavy chain antibodies against type I, IIa, and IIb fibers, oim/oim and WT soleus (sol) and extensor digitorum longus (EDL) muscles were visualized to determine fiber type composition.

Sol and EDL muscles previously stored in optimal cutting temperature (O.C.T.) compound at $-80^{\circ} \mathrm{C}$ were cut to $12 \mu \mathrm{m}$ sections. The primary antibodies mouse IgG2b BA.D5 (1:100), mouse IgG1 SC.71 (1:200), and mouse IgM BF.F3 (1:100) (DSHB, 
University of Iowa, IA) against type I, type IIa, and type IIb fibers respectively were used. Additionally, rabbit IgG anti-laminin antibody (1:200) (ab11575; Abcam) was used to visualize fiber borders. The following Alexa Fluor secondary antibodies were used: 647 goat anti-mouse $\operatorname{IgG} 2 \mathrm{~b}$ (1:250), 488 goat anti-mouse IgG1 (1:500), 555 goat antimouse IgM (1:500), and 350 goat anti-rabbit IgG (1:400) (Invitrogen, IA, USA). Images were captured using the Zeiss Axiovert 200M with fluorescence and ORCA-ER camera and analysis was done using Image $\mathbf{J}$ software.

\section{Glucose Tolerance Testing}

Approximately two weeks prior to sacrifice, mice were fasted for six hours and then injected with $2 \mathrm{mg} / \mathrm{kg}$ glucose. Duplicate glucose measurements were taken and averaged using the ReliOn ${ }^{\mathrm{TM}}$ PRIME (Walmart) glucose monitoring system at the following time points: $0,15,30,60$ and 120 minutes.

\section{Metabolic Chambers and Body Composition Analysis}

Approximately one week prior to sacrifice, mice were placed in metabolic monitoring systems (PromethION; Sable Systems International). Mice were singly housed and given ad libitum access to food (stock chow, Purina Lab Diet 5053) and water. All metabolic chamber data are presented as day and night corresponding to the 12-hour diurnal and nocturnal blocks, respectively. Mice were placed in the chamber on the morning of day 1 and allowed to acclimate until the following morning; data from the subsequent 48 hours, 2 full day and night cycles (days 2 and 3), were analyzed and averaged for each animal. Mice were removed from the chambers on day 4. Data regarding oxygen consumption and carbon dioxide production were collected to determine energy expenditure 
(calculated based on the Weir equation [263]) and respiratory quotient (RQ; calculated as a ratio between oxygen consumption and carbon dioxide utilization [264]) via the PromethION software. RQ gives a sense of fuel utilization with a value of 1 indicative of predominately carbohydrate utilization and a value of 0.7 indicative of predominately fat utilization [264]. Food, water, and body weights were also monitored by the system in addition to activity being measured in three dimensions, in the $\mathrm{x}, \mathrm{y}$, and $\mathrm{z}$ planes, with the $\mathrm{z}$ indicating vertical standing on hindlimbs. All data are presented as absolute values normalized to body weight. Lean body mass and fat mass were determined via EchoMRITM (Houston, USA) prior to mice being placed in the metabolic chamber.

\section{Fat Pad Analysis}

Following sacrifice, the inguinal white adipose tissue, gonadal white adipose tissue, and subscapular brown adipose tissue were removed and weighed as previously described [265].

\section{Serum Markers}

Blood collected via cardiac puncture sat at room temperature for approximately 15 minutes before being placed on ice. All blood was then centrifuged at room temperature in a tabletop centrifuge (15,000 rpm for 15 minutes) and the serum collected and stored at $-80^{\circ} \mathrm{C}$. Serum was then used to measure levels of total glycerol, free glycerol, triglycerides and non-esterified fatty acids (NEFA). Serum total glycerol, free glycerol, and triglycerides (Sigma-aldrich: St. Louis, MO; cat TR0100) as well as NEFA (Wako Diagnostics: 999-34691, 995-34791) levels were determined following the manufacturer instructions. The serum triglyceride kit measures the amount of free glycerol in the serum 
prior to hydrolyzing triglycerides to glycerol and fatty acids at which point the total glycerol is measured again. Triglycerides are determined by subtracting the free glycerol from the total glycerol.

\section{Statistical Analysis}

Statistical analyses were performed using GraphPad Prism 8 program (GraphPad Software, Inc., La Jolla, CA, USA). If not mentioned otherwise, data are presented as mean \pm SE. Male and female data were analyzed independently. For all experiments (minus glucose tolerance tests), an unpaired two-tailed Student's t-test is used for comparisons between two groups if normal distribution of residual was confirmed. For non-normal distributions, Mann-Whitney tests are used for comparisons. For glucose tolerance tests (Figure III-7) we performed two-way repeated measures ANOVA with Geisser-Greenhouse correction for sphericity. Differences with $\mathrm{p}<0.05$ were statistically significant.

\section{Results}

\section{Mitochondrial respiration, citrate synthase activity and fatty acid oxidation}

Previously we demonstrated severe mitochondrial dysfunction in 4-month old male oim/oim gastrocnemius muscle evidenced by reduced gastrocnemius mitochondrial respiration and citrate synthase activity [258] . To determine if this was a global phenomenon or isolated to specific tissues, we investigated mitochondrial respiratory function in the liver and heart as well as gastrocnemius of female oim/oim mice. We found that female oim/oim gastrocnemius mitochondrial respiration was decreased by 27 $45 \%(\mathrm{p}=0.05-0.07)$ with no significant differences in mitochondrial respiration of the 
female oim/oim liver or heart (Figure III-1 A-C) when compared to WT littermates suggesting that mitochondrial dysfunction is tissue specific rather than a global phenomenon. Mitochondrial citrate synthase activity was reduced in female oim/oim gast relative to WT littermates, but did not reach significance. Female liver and heart mitochondrial citrate synthase activity was equivalent between oim/oim and WT littermates (Figure III-1D). To further evaluate the skeletal muscle specificity of mitochondrial dysfunction in the oim/oim mouse, we assessed mitochondrial respiration in 2-month old male mitochondria isolated from both gastrocnemius and quadriceps muscles and saw an overall trend of reduced mitochondrial respiration relative to WT counterparts (Figure III-S1). These findings are consistent with the previous findings in 4-month-old male oim/oim and WT gastrocnemius muscle mitochondria and further support compromised mitochondrial function is a generalized oim/oim skeletal muscle phenomenon that can be present as early as 8 weeks of age. Additionally, we evaluated mitochondrial respiration in heterozygous (+/oim) mice, which exhibit much milder muscle weakness as compared to their oim/oim littermates[3] and found no differences between +/oim gastrocnemius mitochondrial respiration relative to WT littermates (Figure III-S2). 

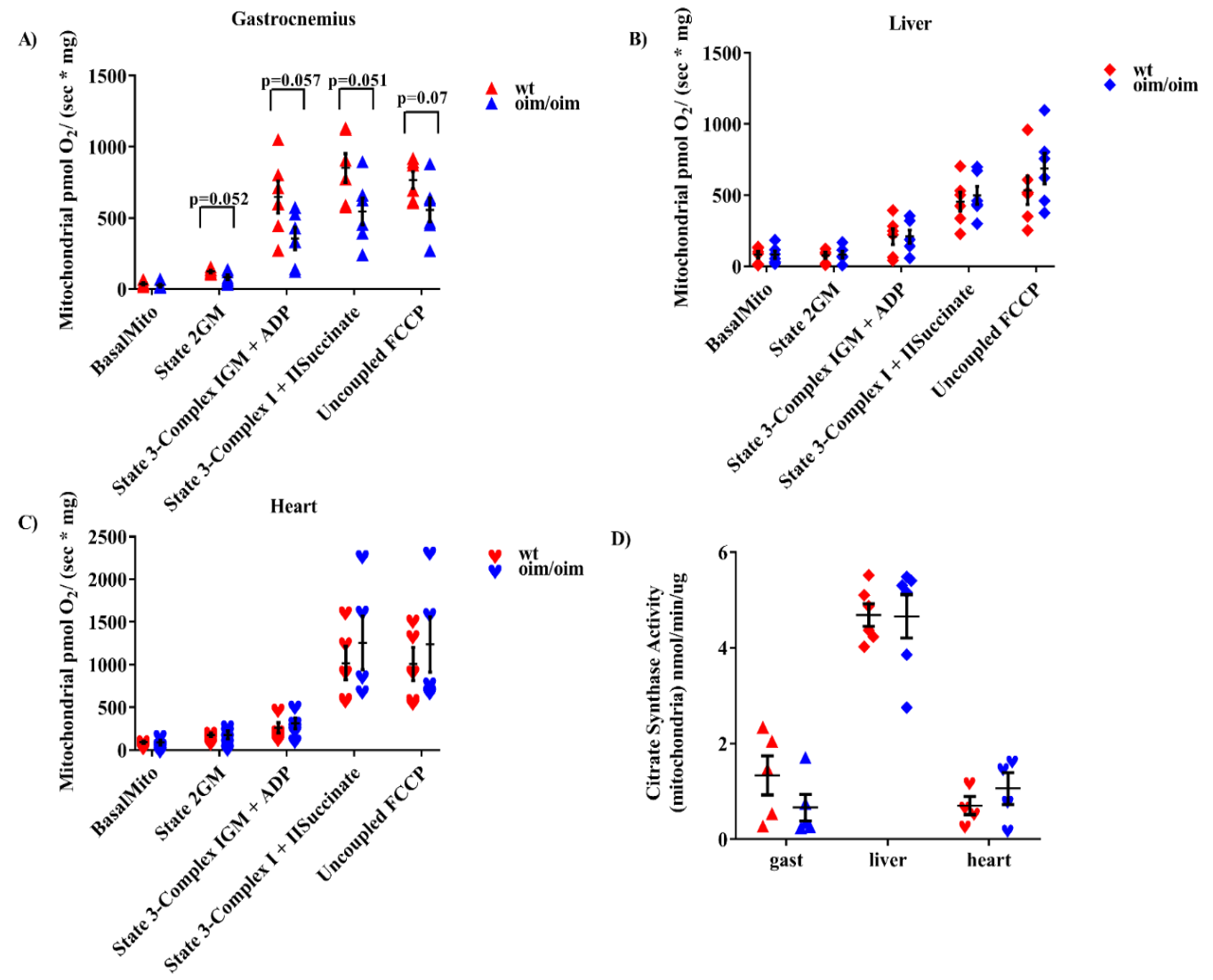

Figure III-1: Isolated mitochondria from female oim/oim (blue) exhibit reduced (A) gastrocnemius (triangles) mitochondrial respiration, while (B) liver (diamonds) and (C) heart (hearts) mitochondrial respiration remains unchanged as compared to isolated mitochondria from WT (red) littermates. (D) There is no difference in isolated mitochondrial citrate synthase activity between female oim/oim and WT gast (triangles), liver (diamonds), or heart (hearts). Data are presented as Means \pm SE. ${ }^{*} \mathrm{p} \leq 0.05$ vs. WT. ( $\mathrm{n}=3-5$ per group). 
Since skeletal muscle mitochondrial function is reduced in oim/oim mice, we assessed fatty acid oxidation (occurring in the mitochondria) of gast muscle mitochondria and whole quad muscle homogenate. The complete palmitate oxidation to $\mathrm{CO}_{2}$ by gast muscle mitochondria tended to be reduced by $60 \%(\mathrm{p}=0.07)$ in male oim/oim mice and was reduced by $85 \%(\mathrm{p}=0.05)$ in female oim/oim mice relative to WT mice (Figure III-2 A), while incomplete and total palmitate oxidation were significantly reduced $(p<0.05)$ in male oim/oim mice only (Figure III-2 B,C). Whole quad muscle homogenate palmitate oxidation was not different between oim/oim and WT littermates regardless of sex (Figure III-2 D-F). 
Isolated gastrocnemius mitochondria
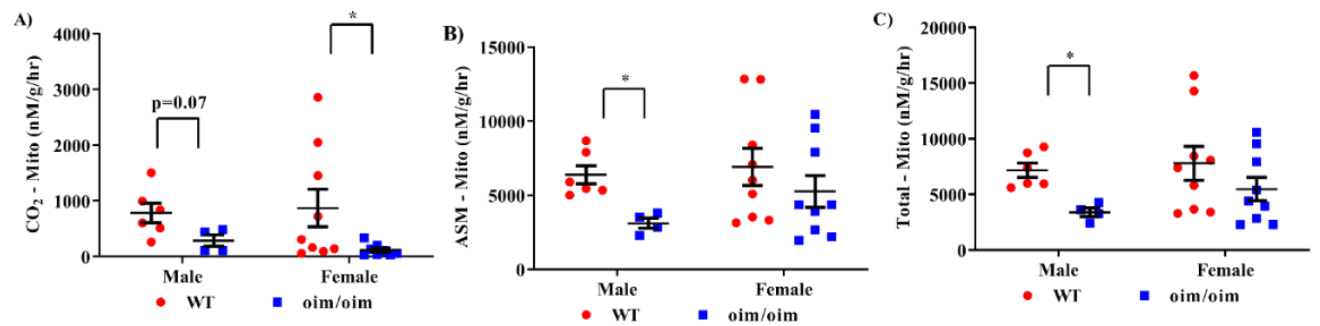

\section{Quadriceps whole muscle homogenate}
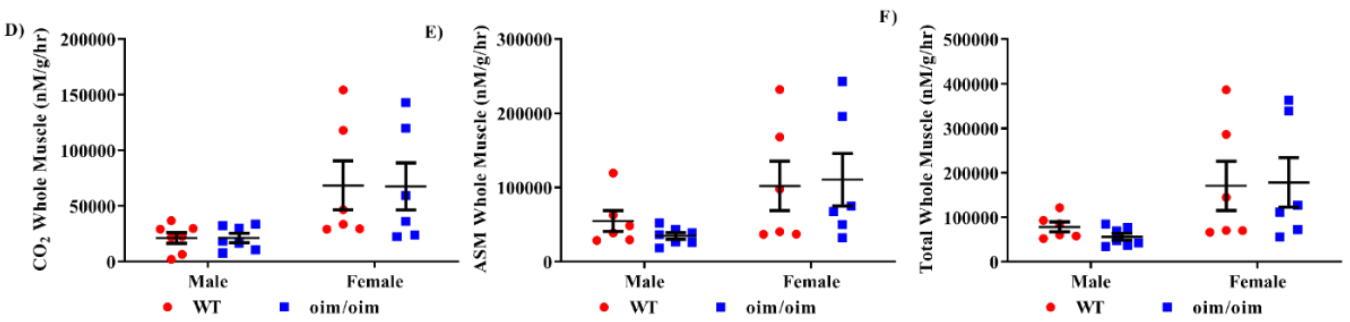

Figure III-2: Isolated mitochondria from male and female oim/oim gastrocnemius (blue squares) exhibit reduced complete oxidation of $\left[1-{ }^{14} \mathrm{C}\right]$-palmitate to $\mathrm{CO}_{2}(\mathrm{~A})$, while male oim/oim mitochondria exhibit reduced incomplete oxidation of $\left[1-{ }^{14} \mathrm{C}\right]$-palmitate to acid soluble metabolites (ASM) (B) and total oxidation of $\left[1{ }^{1-}{ }^{14} \mathrm{C}\right]$-palmitate $\left(\mathrm{ASM}+\mathrm{CO}_{2}\right)(\mathrm{C})$ relative to WT (red circles) littermates. Complete (D), incomplete (E), and total oxidation (F) of $\left[1-{ }^{14} \mathrm{C}\right]$-palmitate in oim/oim whole quadriceps muscle homogenate relative to WT littermates. Data are presented as Means \pm SE. ${ }^{*} \mathrm{p} \leq 0.05$ vs. WT. ( $\mathrm{n}=4-7$ per group). 


\section{Myosin heavy chain analysis of soleus and extensor digitorum longus muscle}

In WT C57BL/6J soleus (sol) muscle the distribution of myofibers is reported to be as follows: $43 \%$ type I, $46 \%$ type IIa, and $11 \%$ type IIx, while the distribution in WT C57BL/6J extensor digitorum longus (EDL) is: 0\% Type I, 11\% Type IIa, and 88\% type IIb [266,267]. Since oim/oim mitochondria are severely dysfunctional [258], and oxidative capacity is largely dependent on mitochondria $[268,269]$, a change in myofiber type composition of the oim/oim skeletal muscle was hypothesized. The size of oim/oim soleus muscles was reduced as was the total number of myofibers $(\mathrm{p}=0.10)$ when compared to WT littermates (Figure III-3C). Male and female oim/oim mice displayed a $33-50 \%$ decrease in the percentage of slow oxidative type I myofibers and a $31 \%$ increase in percentages of fast oxidative type IIa fibers relative to their WT littermates, respectively (Figure III-3 A-B, G-H). In the oim/oim male EDL muscle, type IIa myofibers were increased $129 \%(\mathrm{p}=0.16)$ and type IIb myofibers were decreased $20 \%$ $(\mathrm{p}=0.4)$ relative to WT littermates, although not reaching significance (Figure III-3 D-E, I-J). Total myofiber count was equivalent between oim/oim and WT EDL muscles regardless of sex (Figure III-3F). 


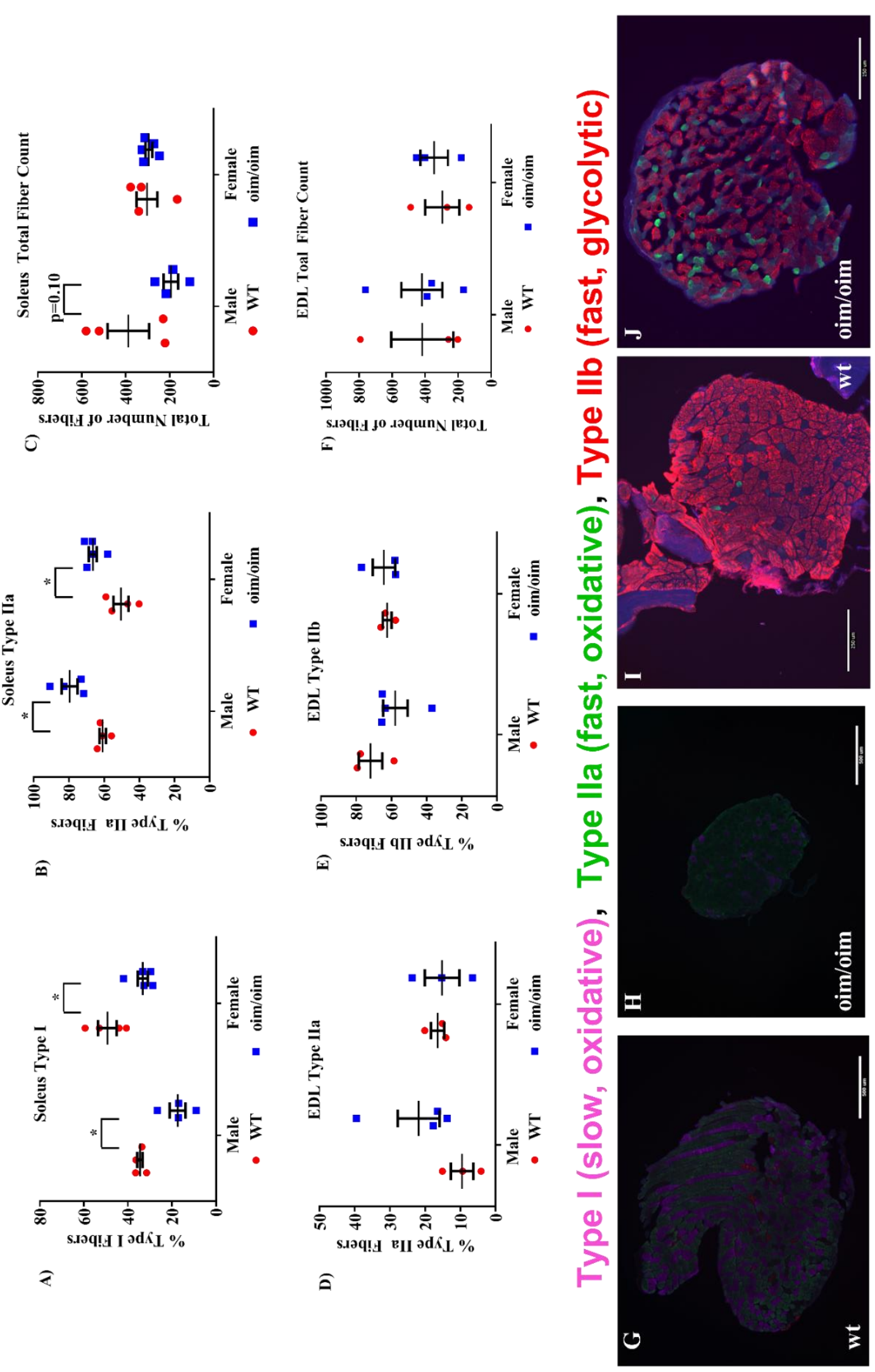


Figure III-3: Male and female oim/oim soleus muscles (blue squares) exhibit reduced percentages of type I myofibers (A) and type IIa myofibers (B) while the total number of myofibers are not altered $(\mathrm{C})$ compared to WT (red circles) littermates. There were no significant changes between WT and oim/oim type IIa myofibers (D), type IIb myofibers (E), or myofiber count (F) in EDL muscles. Representative images of MyHC staining of male WT and oim/oim soleus $(\mathrm{G} \& \mathrm{H})$ and EDL $(\mathrm{I} \& \mathrm{~J})$ muscles. Data are presented as Means \pm SE. ${ }^{*} \mathrm{p} \leq 0.05$ vs. WT. ( $\mathrm{n}=4-5$ per group).

\section{Mitochondrial $\mathrm{H}_{2} \mathrm{O}_{2}$ emission in isolated mitochondria}

Due to the potential role of reactive oxygen species in mitochondrial dysfunction[186], we assessed gastrocnemius mitochondrial $\mathrm{H}_{2} \mathrm{O}_{2}$ emission. In male oim/oim gastrocnemius mitochondria (Figure III-4), we found $\mathrm{H}_{2} \mathrm{O}_{2}$ production was reduced by $32 \%(\mathrm{p}=0.06)$ relative to WT littermates.

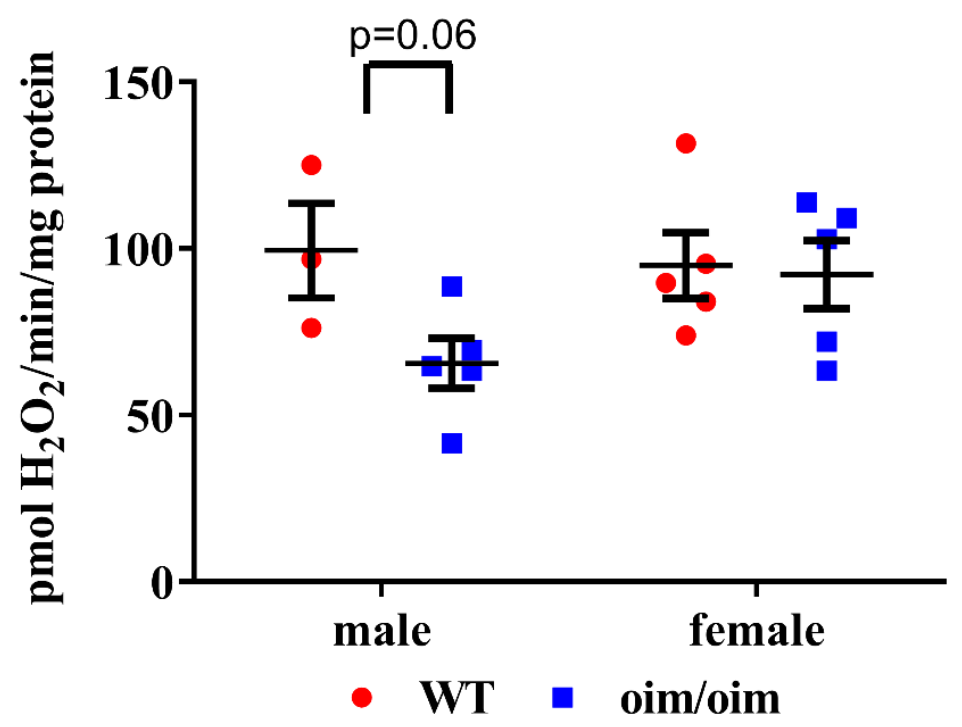

Figure III-4: Isolated male oim/oim (blue squares) gastrocnemius mitochondria produced reduced amounts of $\mathrm{H}_{2} \mathrm{O}_{2}$ when stimulated with palmitoyl-CoA compared to WT isolated gastrocnemius mitochondria (red circles) littermates. Data are presented as Means \pm SE. ${ }^{*} \mathrm{p} \leq 0.05$ vs. WT. ( $\mathrm{n}=3-5$ per group). 


\section{Body composition}

By EchoMRI, male oim/oim mice were determined to have a 3\% ( $\mathrm{p}=0.12)$ increase in lean mass and a 20\% $(\mathrm{p}=0.07)$ decrease in fat mass relative to WT littermates (Figure III5 A-B). Female oim/oim mice did not exhibit alterations fat or lean mass relative to female WT littermates. Both male and female oim/oim mice exhibited reduced relative gonadal white adipose tissue (although only reaching significance in female mice) while relative inguinal white adipose tissue was equivalent between oim/oim and WT littermates regardless of sex (Figure III-5 C-D). Subscapular brown adipose tissue (BAT) was not different between WT and oim/oim animals. 

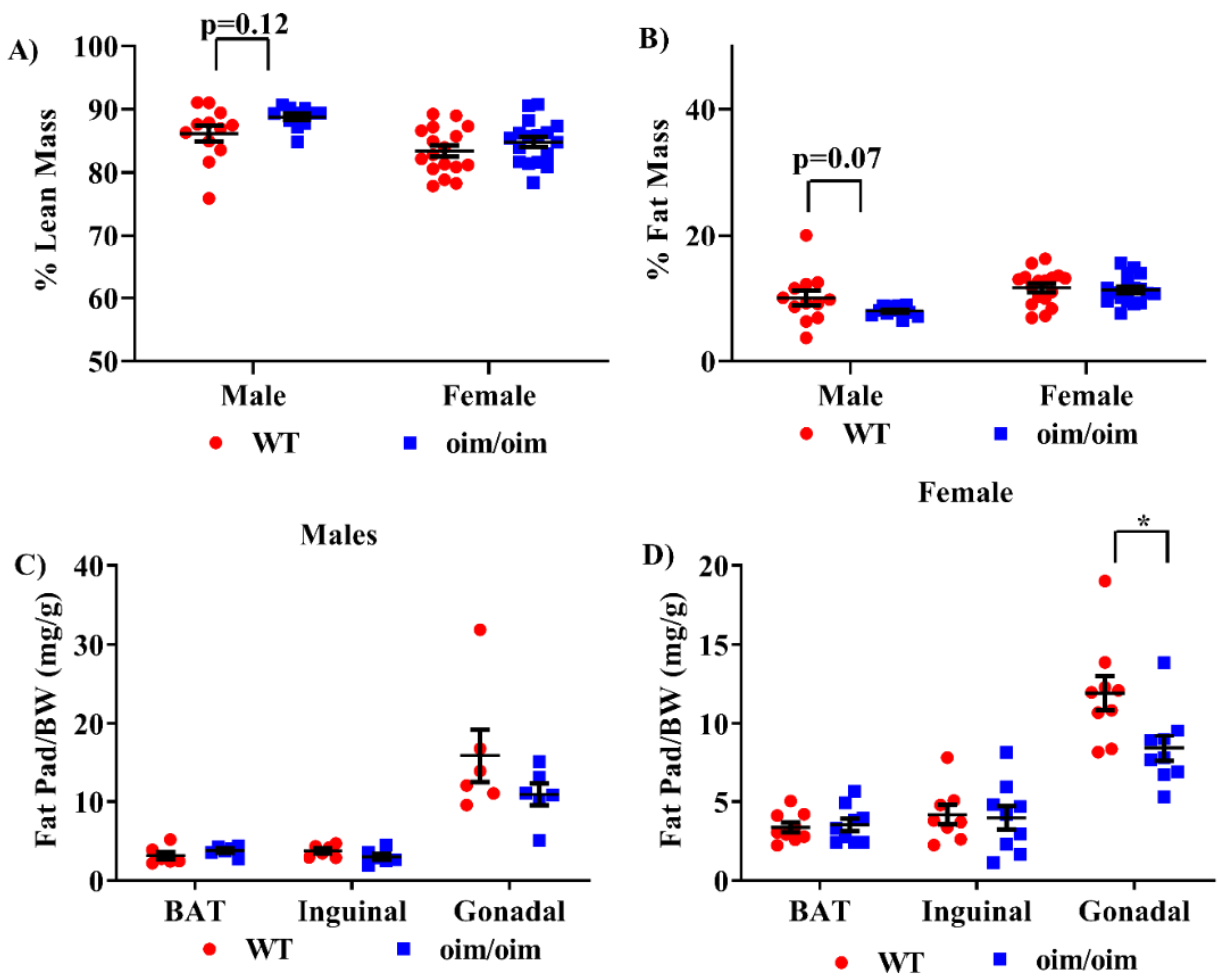

Figure III-5: Male oim/oim (blue squares) mice exhibit increased percentages of lean mass (A) and reduced percentages of fat mass (B) compared to WT (red circles) littermates. Additionally, relative gonadal fat pad weights were reduced in female oim/oim mice while brown adipose tissue and inguinal adipose depots remained unchanged in oim/oim mice compared to WT littermates (C-D). Data are presented as Means \pm SE. ${ }^{*} \mathrm{p} \leq 0.05$ vs. WT. $(\mathrm{n}=11-17$ per group $)$. 


\section{Indirect calorimetry}

WT and oim/oim mice were placed in metabolic chambers to determine their whole body energy metabolism; parameters measured included: energy expenditure, activity, and food/water consumption. When normalized to body weight, oim/oim mice displayed significantly increased energy expenditure in light and dark cycles (+9-19\%), respectively (Figure III-6 A-D). This occurred despite a decrease in physical activity of female and male oim/oim relative to sex-matched WT mice during both light and dark cycles (Figure III-S3). Additionally, male oim/oim mice consumed $\sim 36 \%$ more food per body weight during the 12 hour night cycle compared to their WT littermates $(\mathrm{p}=0.06$; Figure S4). 

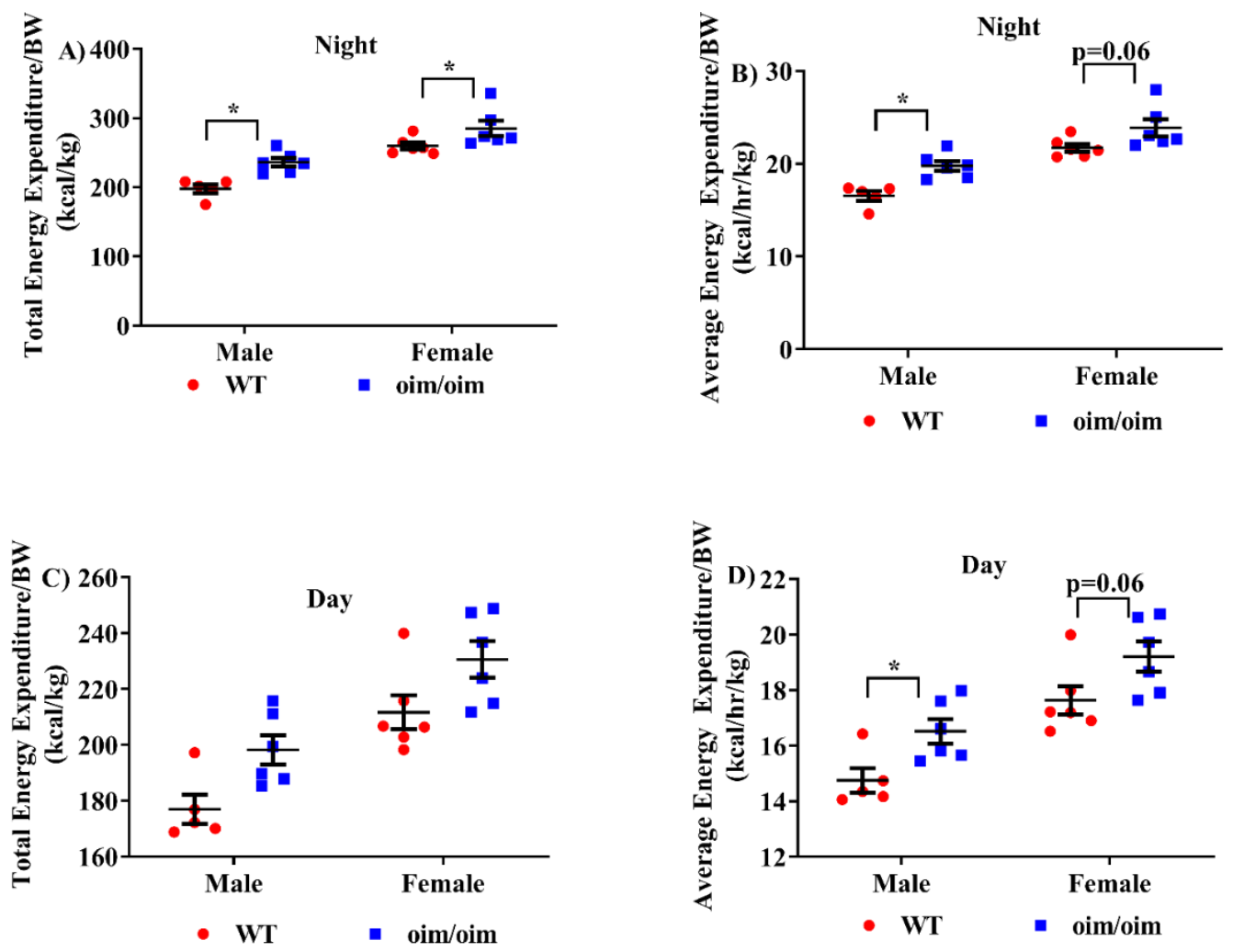

Figure III-6: When normalized to body weight, both male and female oim/oim (blue squares) mice exhibit increased total $(\mathrm{A}, \mathrm{C})$ and average $(\mathrm{B}, \mathrm{D})$ energy expenditures for the night $(\mathrm{A}, \mathrm{B})$ and day $(\mathrm{C}, \mathrm{D})$ cycles when compared to WT (red circles) littermates. Data are presented as Means \pm SE. ${ }^{*} \mathrm{p} \leq 0.05$ vs. WT. ( $\mathrm{n}=5-6$ per group). 
During both day and night cycles, oim/oim animals consumed significantly greater amounts of oxygen per body weight (+9-20\%) compared to their WT littermates (Figure III-S5 A\&C), and oim/oim mice produced significantly greater amounts of carbon dioxide per body weight (+6-18\%) relative to WT littermates during the night cycle (Figure IIIS5 B\&D). The respiratory quotient was not altered in oim/oim mice relative to WT littermates (Figure III-S5 E\&F). Although, high variation and low sample number have likely limited the power of this analysis. 


\section{Glucose Tolerance}

Despite reduced skeletal muscle mitochondrial respiration (Figure III-1 \& S1) and fatty acid oxidation (Figure III-2), glucose tolerance was not different in oim/oim mice compared to WT littermates (Figure III-7).

A)

\section{Males}

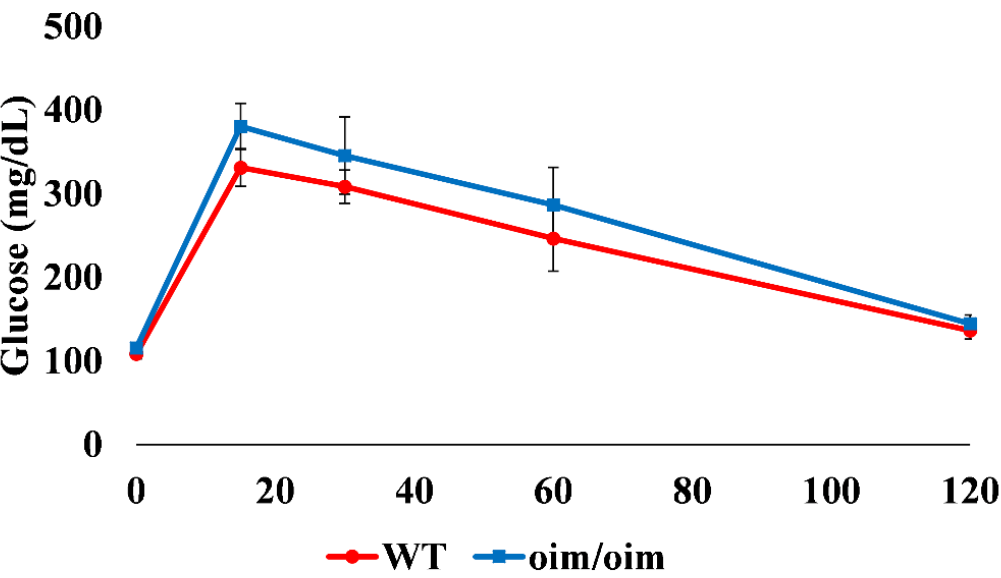

B)

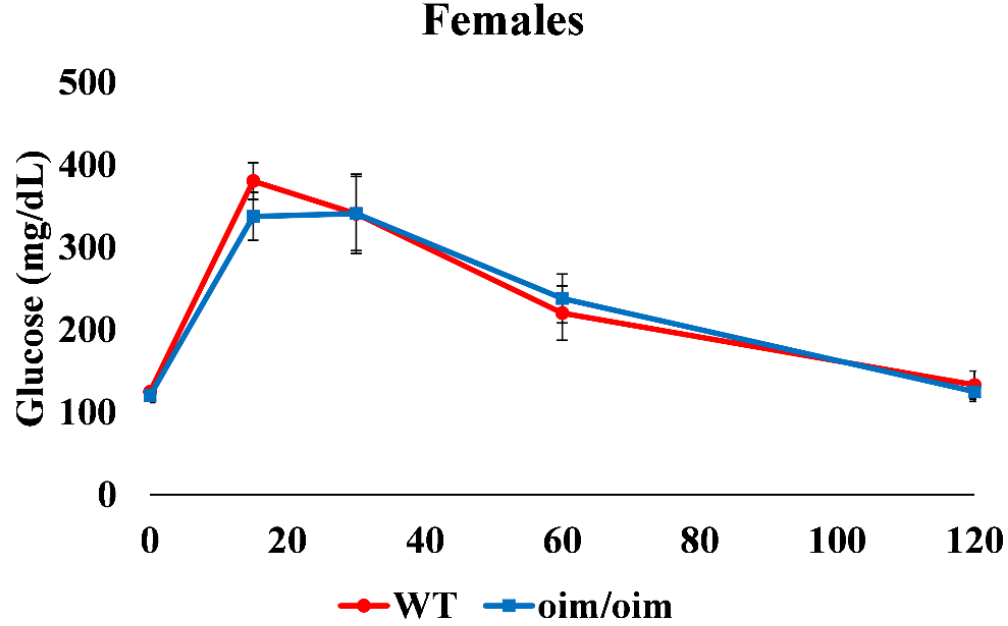

Figure III-7:

oim/oim (blue

squares) mice do not exhibit changes in glucose tolerance compared to WT (red circles) littermates. Male (A) and female (B) mice were fasted for six hours, then injected with 2 mg glucose per gram of body weight, and glucose measurements taken at the following time points: 0,15 , 30,60 , and 120 minutes. Data are presented as Means $\pm \mathrm{SE}$ $(\mathrm{n}=6$ per group). 


\section{Serum markers}

While serum triglycerides and NEFAs did not differ between oim/oim and WT

littermates, serum free glycerol was drastically reduced in male oim/oim mice (-68\%)

relative to WT littermates (Figure III-8).
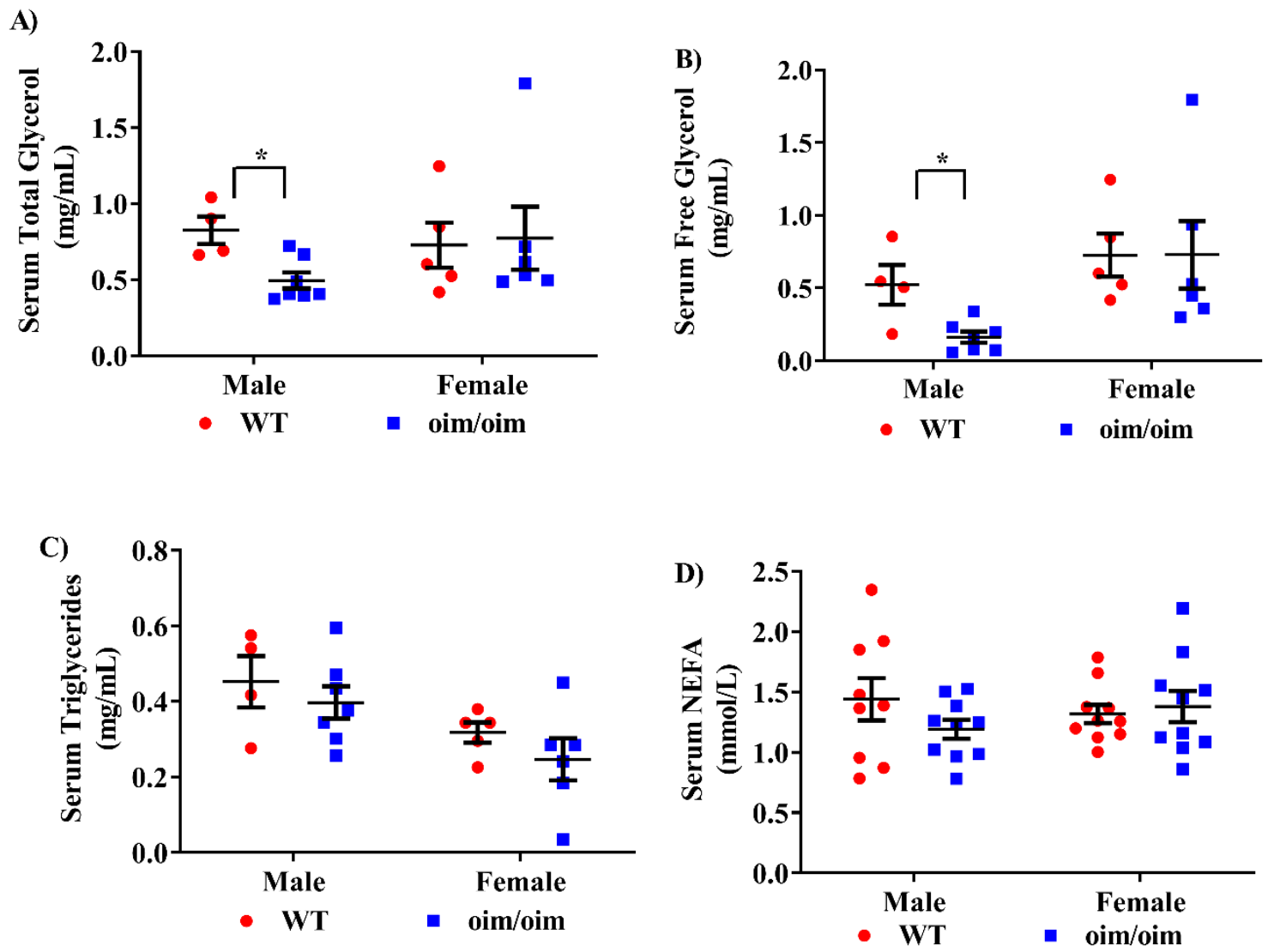

Figure III-8: Male oim/oim mice (blue squares) exhibit reduced total glycerol (A) and free glycerol levels in serum (B) compared to WT red circles) littermates while no differences were present in serum levels of triglycerides $(C)$ and free fatty acids (NEFA) (D) between oim/oim and WT littermates. Data are presented as Means \pm SE of the genotype values. ${ }^{*} \mathrm{p} \leq 0.05$ vs. WT. ( $\mathrm{n}=4-10$ per group). 


\section{Discussion}

Skeletal muscle weakness is extremely prevalent in OI where roughly $80 \%$ of patients with mild to moderate types I and IV OI experience muscle force deficits and even higher levels in more severe patients as muscle weakness in OI correlates with the level of ambulation $[4,6,48,115,207]$. Bone and muscles are highly synergistic tissues that talk to one another via both biochemical signaling and mechanotransduction [57,59,257]. Thus, investigating the mechanisms by which skeletal muscle weakness arises is essential.

Previously, we found that skeletal muscle mitochondrial dysfunction was implicated as part of the pathophysiology of OI in the oim/oim mouse model [258]. Here, we further evaluated the impact and potential links between mitochondrial dysfunction, skeletal muscle weakness and whole-body metabolism. We demonstrate that the oim/oim mitochondrial dysfunction appears tissue specific manifesting in skeletal muscle (gastrocnemius and quadriceps) rather than globally as respiration in heart and liver mitochondria were not compromised in oim/oim mice. Additionally, we found alterations in skeletal muscle and whole body parameters related to energy metabolism.

Skeletal muscle, constituting roughly $50 \%$ of body mass, is a significant contributor to the body's resting metabolic rate $[55,270,271]$. Yet, despite countless reports of skeletal muscle dysfunction in OI patients, there is a paucity of studies on the role of energy metabolism in OI $[4,6,48]$. Reports in prepubescent patients and the Collal ${ }^{\mathrm{Jt} / \mathrm{+}}$ mouse suggest a metabolic phenotype in the pathophysiology of OI[7,8]. Reports in prepubescent patients suggest a hyper-metabolic phenotype consisting of increased body temperature, heart rate, and respiratory rates [7] while a more recent report by Boraschi- 
Diaz et al. implicates whole-body metabolic changes in young mice (4 weeks), including reduced adiposity, increased energy expenditure, increased oxygen consumption, and increased carbon dioxide production, as part of the pathophysiology of OI in the Collal $^{\mathrm{Jt} / \mathrm{+}}$ mouse model, containing an 18 amino acid deletion in the triple helical domain of the pro- $\alpha 1(\mathrm{I})$ collagen chain $[8,51]$. Similarly, our observations also implicate a metabolic phenotype in the oim/oim mouse as evidenced by increased relative lean mass and reduced fat mass (Figure III-5 A-B), increased energy expenditure despite reduced activity (Figure III-6 and Figure III-S3), and increased oxygen consumption and carbon dioxide production (Figure III-S5). Although, the metabolic phenotype observed in the Collal $1^{J r t /+}$ mouse was diminished by 8 weeks of age, in the oim mouse this was observed in adult oim/oim mice aged 16-19 weeks.

Despite increased energy expenditures in the oim/oim mouse, we did not observe changes in the respiratory quotient, glucose tolerance, or skeletal muscle fatty acid oxidation suggesting that the oim/oim mice do not exhibit shifts in substrate utilization in response to altered energy metabolism. Additionally, we measured total glycerol, free glycerol, triglyceride, and NEFA levels in serum and found that while serum glycerol was the only marker significantly reduced, triglyceride and NEFA markers also appear slightly decreased in the oim/oim male mouse compared to WT littermates (Figure III-8). Glycerol, an important gluconeogenic substrate secreted by white adipose tissue [272,273], was shown to be increased in Swedish men with type II diabetes [274]. The decrease in serum glycerol seen in the oim/oim mouse relative to WT littermates is likely associated with the reduced relative fat mass and may be indicative of upregulated 
gluconeogenesis, although further studies to better understand the nature of this change are warranted.

Mitochondrial function and content exhibit tissue diversity due to differing cellular needs with cardiac tissue mitochondria having the highest electron transport chain activity relative to either liver or gastrocnemius mitochondria[275-277]. The tissue specific mitochondrial dysfunction observed in the oim/oim mouse may reflect differing tissue response to the type I collagen defect.

Skeletal muscle mitochondrial dysfunction may also reflect the observed change in fiber type distribution observed in the soleus muscle. The soleus muscle, containing the slow oxidative and fast oxidative fibers (type I and IIa, respectively) is considered mitochondria-rich, whereas the extensor digitorum longus (EDL) muscle containing types IIa and IIb fibers (representing fast oxidative and fast glycolytic, respectively), has more variable mitochondrial content[278]. Both male and female oim/oim mice exhibited decreased percentages of type I fibers and increased percentages of type IIa fibers in the soleus muscle relative to WT littermates (Figure III-3). Oim/oim male EDL muscles exhibited increased type IIa fibers and decreased IIb fibers relative to WT littermates, (although not reaching significance), while female fiber type distribution was equivalent between WT and oim/oim (Figure III-3). The decrease in type I fibers of the soleus muscle may serve as a compensatory mechanism for the dysfunctional mitochondria. Moreover, the contribution of reduced activity levels in oim/oim mice (Figure III-S3) to fiber type changes in addition to mitochondrial function cannot be discounted, as activity serves as important regulator of fiber type composition and mitochondrial bioenergetics[279,280]. 
Reduced activity levels in oim/oim mice have been previously reported[163], but the cause(s) of this reduced activity remain unknown. However, the increased prevalence of fractures in the oim/oim mice is potentially an important contributor to decreased activity levels. Specifically, increased long bone fracture rates of oim/oim mice [39] could potentially explain the reduced rearing activity in the z plane that was observed. It is important to note that skeletal muscle weakness in the oim/oim mouse is intrinsic, as demonstrated by the reduced specific contractile force (muscle contractile force normalized to muscle cross sectional area), suggesting that reduced activity alone is not solely responsible for the muscle weakness[3].

In our current study, we observed that skeletal muscle mitochondrial fatty acid oxidation in isolated oim/oim gastrocnemius mitochondria was reduced, but it was not different in whole quadriceps muscle homogenates relative to WT littermates (Figure III-2). This suggests that the reduction in mitochondrial fatty acid oxidation may be the result of dysfunctional mitochondria, as mitochondria are the primary site of fatty acid betaoxidation [281]. The fact that fatty acid oxidation in the whole muscle was not altered suggest that there may be an increased number of mitochondria in the oim/oim skeletal muscle as proposed previously [258], which could lead to a compensation effect. Additionally, we evaluated skeletal muscle mitochondrial ROS production. Although we hypothesized increased hydrogen peroxide as a contributor to mitochondrial dysfunction, we found that hydrogen peroxide emission per amount of mitochondria was reduced in male oim/oim gastrocnemius, which may again be the result of poorer functioning mitochondria (Figure III-4). Future studies are warranted to determine the amount of hydrogen peroxide produced within the whole muscle. 
In this study, we provide further evidence that the oim/oim mouse exhibits tissue specific skeletal muscle mitochondrial dysfunction independent of heart and liver tissue as evidenced by changes in skeletal muscle mitochondrial respiration, mitochondrial fatty acid oxidation, and mitochondrial ROS production. Additionally, we suggest that a metabolic phenotype exists in the oim/oim mouse that likely contributes further to the pathophysiology of the disease as evidenced by increases in energy expenditure and altered body composition. Further studies are warranted to determine the nature of the relationship between a type I collagen mutation, mitochondrial defects, and metabolic alterations. Understanding these relationships could lead to novel therapeutics addressing the muscle weakness and bone fragility associated with osteogenesis imperfecta.

\section{Limitations}

The limitations of this study include the age of the mice, which were primarily from 13 weeks-19 weeks with a subset at 8 weeks. Narrower age range or an earlier prepubescent age may provide additional information concerning the relationship between metabolic alterations and metabolism during the most vulnerable fracture time period [282]. An additional limitation includes small animal number for certain analyses, including metabolic cages and fatty acid oxidations, which had to be balanced against time and fiscal costs.

Authors' roles: Study design: VLG, LCS, CLP, and RSR. Study conduct: mitochondrial isolation and mitochondrial experiments/respiration (VLG), fatty acid oxidation experiments (VLG), glucose tolerance testing (VLG, EH), metabolic chambers (VLG), fiber type experiments (VLG, EH, TKC), serum measurements (VLG). Data analysis: 
VLG, EH, TKC, AO, LCS, RSR, and CLP. Drafting manuscript: VLG, EH, TKC, AO, LCS, RSR, and CLP. Revising manuscript content: VLG, EH, TKC, AO, LCS, RSR, and CLP. Approving final version of the manuscript: VLG, EH, TKC, AO, LCS, RSR, and CLP. CLP takes responsibility for the integrity of the data analysis. 


\section{Supplementary Material for Chapter III}

Figure III-S1: Isolated gastrocnemius (A) and quadriceps (B) muscle mitochondria from 2 month old male oim/oim (blue squares) mice exhibit reduced mitochondrial respiration when compared to WT littermates (red circles). Mitochondrial respiration was measured using the Oroboros Oxygraph-2k (Oroboros Instruments, Innsbruck, Austria) as described in the methods. Data are presented as Means \pm SE. ( $n=3-5$ per group).
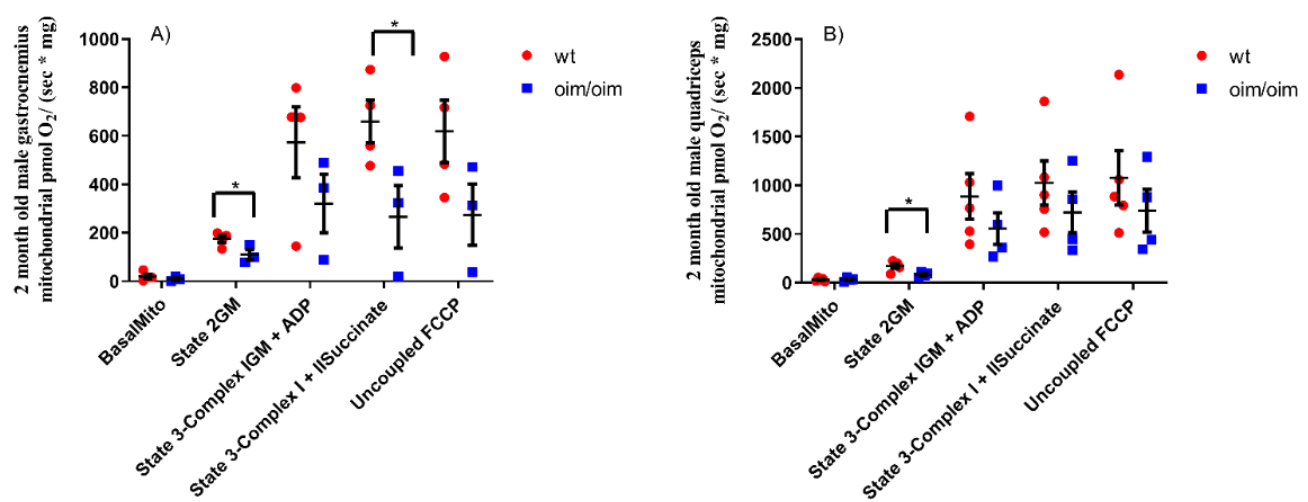
Figure III-S2: Isolated gastrocnemius muscle mitochondria from female heterozygous +/oim (orange triangles) mice do not exhibit mitochondrial dysfunction when compared to WT littermates (purple circles). Gastrocnemius mitochondrial respiration was measured using the Oroboros Oxygraph-2k (Oroboros Instruments, Innsbruck, Austria) as described in the methods. Data are presented as Means \pm SE. ( $n=5-6$ per group).

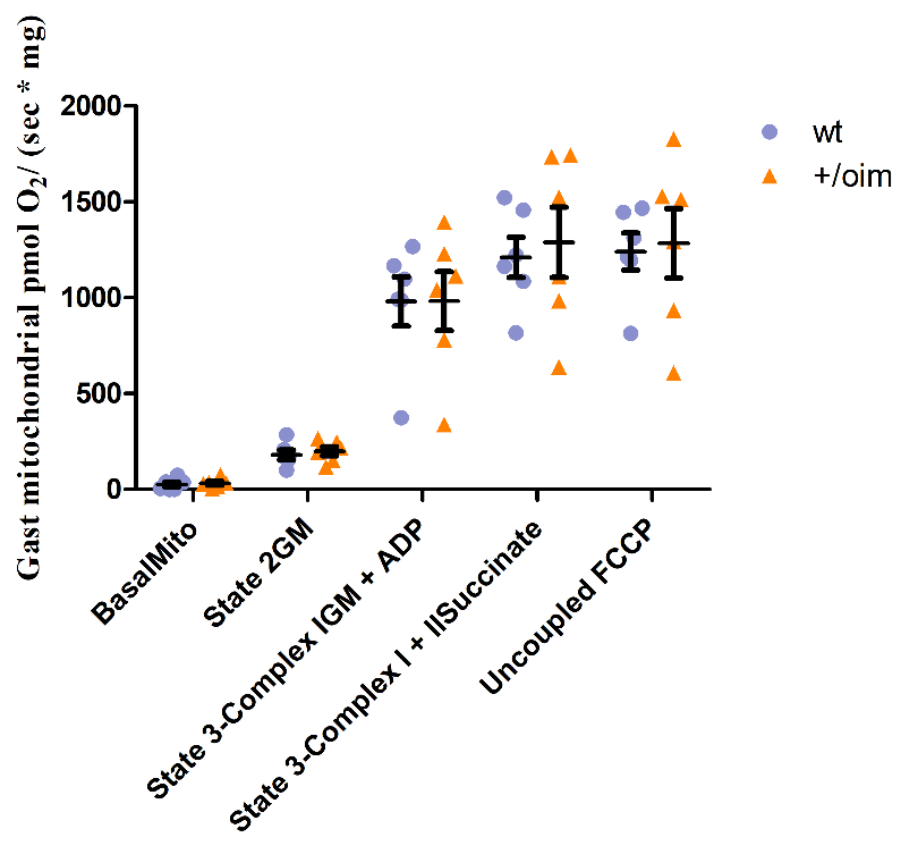


Figure III-S3: Male oim/oim (blue squares) mice exhibit altered activity levels during the night $(\mathrm{A})$ and day $(\mathrm{C})$ cycles while female oim/oim mice only exhibit changes in $\mathrm{Z}$ breaks (rearing) during the day (D) cycle and exhibit no changes during the night cycle (B) when compared to WT (red circles) littermates. Activity levels were assessed using metabolic monitoring systems (PromethION; Sable Systems International). Data are presented as Means \pm SE. ${ }^{*} \mathrm{p} \leq 0.05$ vs. WT. ( $\mathrm{n}=5-6$ per group).
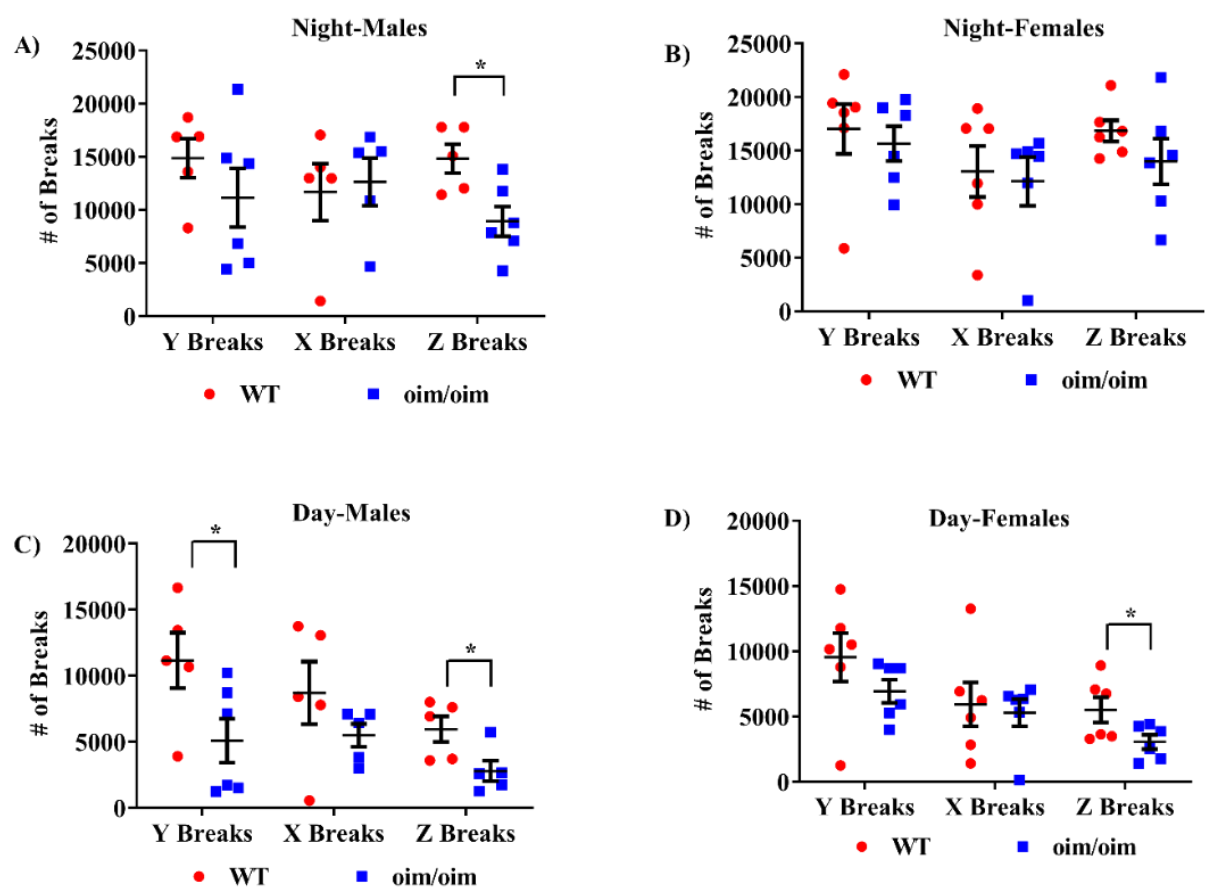

Figure III-S4: Male oim/oim (blue squares) mice exhibit increased food consumption with no changes in water consumption while female oim/oim mice do not exhibit changes 
in their eating or drinking habits during either the night (A-B) or day (C-D) cycles compared to WT (red circles) littermates. Food and water consumption were assessed using metabolic monitoring systems (PromethION; Sable Systems International). Data are presented as Means \pm SE. ${ }^{*} \mathrm{p} \leq 0.05$ vs. WT. ( $\mathrm{n}=5-6$ per group).
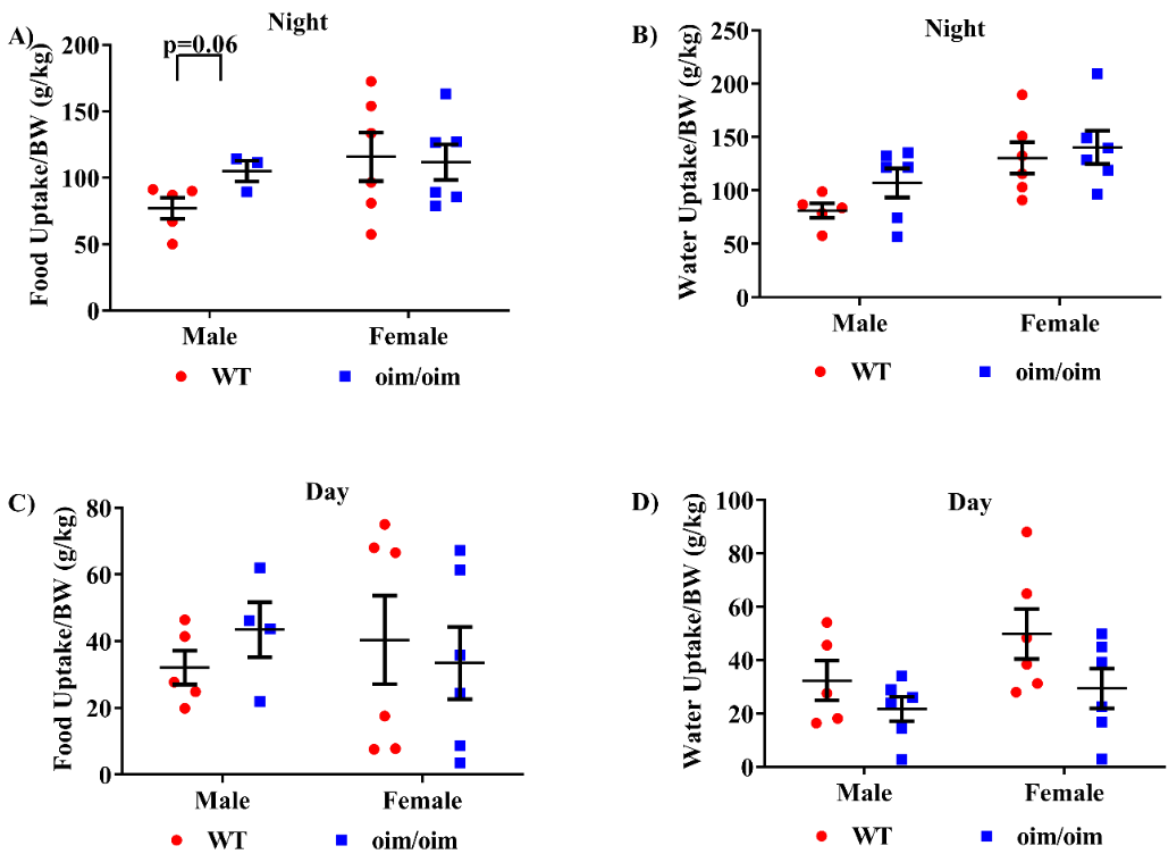
Figure III-S5: When normalized to body weight, both male and female oim/oim (blue squares) exhibit increased $\mathrm{V}_{\mathrm{O} 2}$ levels for both night and day cycles $(\mathrm{A}, \mathrm{C})$ while $\mathrm{V}_{\mathrm{CO} 2}$ levels increased during the night $(\mathrm{B}, \mathrm{D})$ cycle only, relative to WT littermates (red circles). The respiratory quotient, the ratio of carbon dioxide produced to oxygen consumed, remained unchanged during both the night and day cycles (E-F) compared to WT littermates. Oxygen consumption and carbon dioxide production were assessed using metabolic monitoring systems (PromethION; Sable Systems International). Data are presented as Means \pm SE. ${ }^{*} \mathrm{p} \leq 0.05$ vs. WT. ( $\mathrm{n}=5-6$ per group).
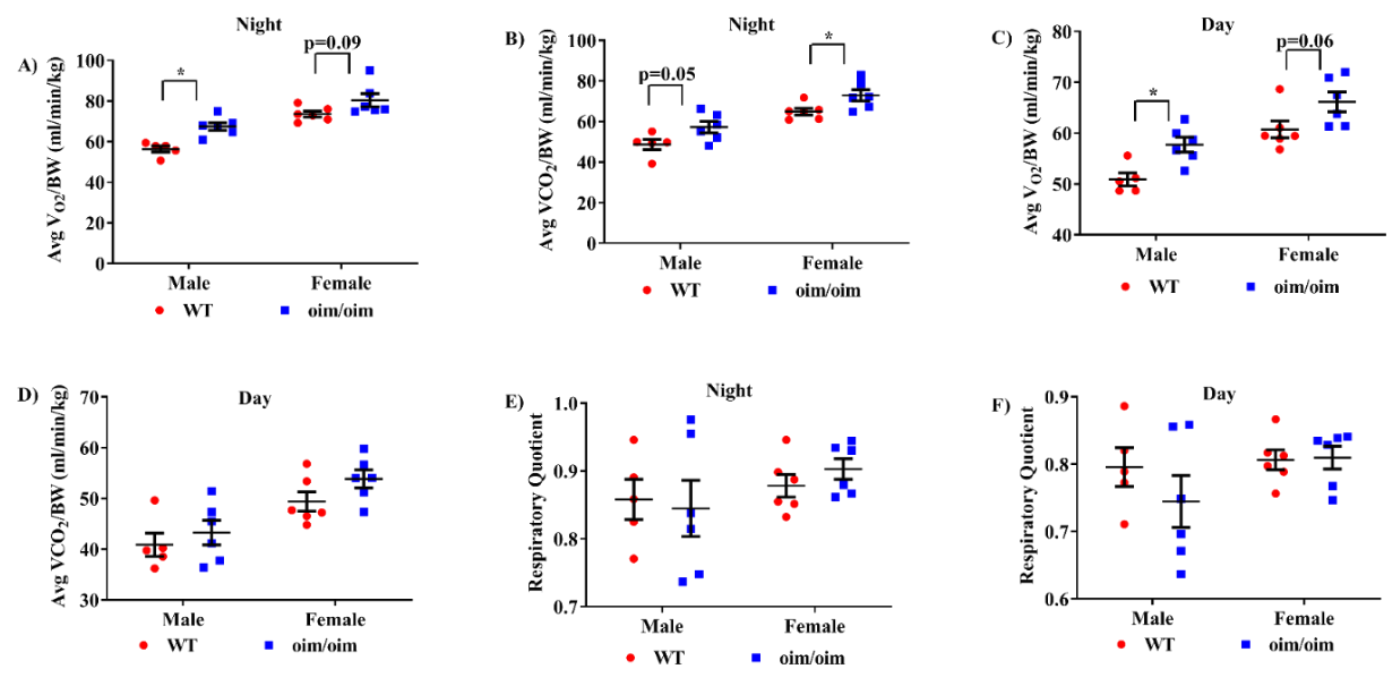


\section{CHAPTER IV}

\section{PERSPECTIVES AND FUTURE DIRECTIONS}


The aims of this research were to 1) characterize mitochondrial function in the hindlimb muscles of a mouse modeling a severe patient type III OI (oim/oim), 2) determine if mitochondrial dysfunction is a global phenomenon in the oim/oim mouse, and 3) determine if whole body and skeletal muscle metabolic parameters are altered in the oim/oim mouse. To this end, we discovered that 1) oim/oim mice exhibit severe skeletal muscle mitochondrial dysfunction evidenced by drastic reductions in mitochondrial respiration and mitochondrial citrate synthase activity, 2) this mitochondrial dysfunction is not global as reduced mitochondrial respiration is not observed in heart or liver tissue, and finally, 3) a subtle metabolic phenotype is present in the oim/oim mice including the presence of altered body composition and increased energy expenditure.

These studies were the first to demonstrate the presence of mitochondrial dysfunction in an OI mouse model. However, much more work is needed to fully understand the implications of these findings. The following three objectives outline the important future studies that are necessary to better understand these findings and to move the field forward.

\section{Objective 1}

Evaluate the role of the extracellular matrix and mitochondrial permeability transition pore induction on mitochondrial dysfunction and skeletal muscle weakness in the oim/oim mouse. 
Hypothesis: The presence of abnormal type I collagen disrupts the interactions and organization within the extracellular matrix of skeletal muscle leading to mitochondrial dysfunction and skeletal muscle weakness in the oim/oim mouse.

Rationale: Type I collagen is the most abundant collagen in both the tendon and perimysium of the skeletal muscle while types I and III collagens are equally abundant in both the epi- and endomysium[92]. The organization of type I collagen differs from tendon and perimysium with the type I collagen in tendon being much more organized than in the perimysium. This likely reflects the different functions of each component[92]. This highly organized hierarchical extracellular matrix surrounding the muscle fibers is believed to help facilitate force transduction with all the different hierarchical levels believed to be interconnected mechanically[92,94]. Oim/oim mice exhibit abnormal type I collagen existing as a homotrimer composed of three alpha I chains, $\alpha 1(\mathrm{I})_{3}$, rather than normal heterotrimer $\left[\alpha 1(\mathrm{I})_{2} \alpha 2(\mathrm{I})\right][39]$. Additionally, deficits of type VI collagen, an important and abundant extracellular matrix protein found in skeletal muscle, results in two classical myopathies, the milder Bethlem myopathy and the more severe Ullrich congenital muscular dystrophy, both characterized by muscle weakness and hypotonia, distal joint laxity, and proximal joint contractures [180]. Type VI collagen monomers are triple helixes composed of one $\alpha 1$ (VI), $\alpha 2$ (VI), and $\alpha 3$ (VI) chain (occasionally the $\alpha 3$ (VI) chain is replaced by an $\alpha 4$ (VI), $\alpha 5$ (VI), or $\alpha 6$ (VI) chains) [195]. These triple helical monomers form disulfide bonded dimers, and ultimately disulfide bonded tetramers giving type VI collagen a characteristic beaded microfibril pattern [195]. Collagen VI deficiencies in mouse models also lead to skeletal muscle weakness and mitochondrial dysfunction which is hypothesized to arise as a result 
dysregulated calcium signaling and ultimately increased induction of the mitochondrial permeability transition pore [181]. The exact structure of the mitochondrial permeability transition pore has yet to be elucidated, however there is evidence implicating cyclophilin $\mathrm{D}$, a prolyl isomerase, as a regulator of the mitochondrial permeability transition pore.

Furthermore, types I and VI collagens have been shown to interact with one another in vitro [283]. Moreover, there is evidence suggesting the interaction between types I, VI, III, and XII collagens in the organization of the perimysium[205]. This organization, especially at the sites where the perimysium converges with the endomysium, a region termed the perimysial junction plate, is believed to contribute significantly to lateral force transmission and ultimately to contractile forced [205]. This link between abnormal type VI collagen and mitochondrial dysfunction leads us to question the role of collagen interactions, such as those of type I and type VI collagen, within the extracellular matrix on skeletal muscle and mitochondrial function.

Approach: We will assess induction of the mitochondrial permeability transition pore, indirectly, in gastrocnemius muscle of the oim/oim mouse by measuring calcium retention capacity of isolated mitochondria, as excessive calcium is a known inducer of mitochondrial permeability transition. Furthermore, we will quantify cyclophilin D protein content, another known regulator of mitochondrial permeability transition, via western blot. Additionally, we will measure the gene expression and protein content of type VI collagen; specifically, we will measure these parameters in the $\alpha 1, \alpha 2$, and $\alpha 3$ chains of type VI collagen. We will evaluate the relationship of homotrimeric type I collagen and normal type VI collagen as compared to normal heterotrimeric type I collagen and type VI collagen by assessing binding strength via microscale 
thermophoresis in conjunction with the University of Missouri Molecular Interactions

Core.

\section{Objective 2}

Determine if skeletal muscle weakness and mitochondrial dysfunction are inherent to the oim/oim mouse or acquired as the mice age, as a consequence of inactivity or some other factor.

Hypothesis: Abnormal type I collagen in the oim/oim mouse leads to an inherent skeletal muscle weakness and mitochondrial dysfunction that is present at the neonate stage and independent of inactivity.

Rationale: Previous work demonstrates that oim/oim mice exhibit reduced specific contractile generating force (contractile generating force normalized to myofiber cross sectional area) suggesting that these mice exhibit skeletal muscle weakness that is independent of other factors that can lead to reduced function as a result of reduced muscle size, such as inactivity. Furthermore, oim/oim mice exhibit reduced activity levels as early as one month of age, and this inactivity may be the result of reduced skeletal muscle weakness.

Approach: Using primary myocytes cultured from 7 day old neonatal oim/oim and WT hindlimb muscles, we will assess myotube diameter and contractile function using electric pulse stimulation as previously described[284]. Additionally, mitochondrial function will be assessed using the Seahorse XF Analyzer to measure mitochondrial respiration in myocytes as previously described[285]. 


\section{$\underline{\text { Objective } 3}$}

Evaluate the role of OI mutation in addition to the relationship between skeletal muscle and mitochondrial function using three distinct mouse models of OI (+/G610C, Collal $1^{\mathrm{Jt} / \mathrm{+}}$, and CRTAP $\left.^{-/-}\right)$.

Hypothesis: Mice modeling more severe forms of OI (Collal ${ }^{\mathrm{Jt} / \mathrm{+}}$ and $\left.\mathrm{CRTAP}^{-/-}\right)$, similarly to the oim/oim mouse, will exhibit compromised skeletal muscle function and reduced mitochondrial function while mice with less impacted muscle function $(+/ G 610 C)$ will not exhibit as poor mitochondrial function.

Rationale: Recent evidence of mitochondrial dysfunction in the oim/oim mouse, including altered mitochondrial number and reduced mitochondrial function, in conjunction with reduced contractile generating force within skeletal muscle suggests mitochondrial dysfunction plays a role in the pathogenesis of OI muscle weakness and warrants the investigation of mitochondrial function in other mouse models of OI exhibiting different mutations and levels of severity[3,258]. +/G610C mice model a mild human type I/IV OI due to a COL1A2 gene variant (a glycine to cysteine substitution) identified in an extensive Old Order Amish kindred of 64 OI individuals in 37 nuclear families[50]. The Collal $1^{\text {Jrt/+ }}$ mouse is the first combined model of both severe type IV OI and Ehlers Danlos Syndrome due to a COL1A1 mutation leading to skipping of exon 9 and subsequent 18 amino acid deletion in the triple helical domain[51]. The CRTAP $P^{-/}$ mice model a recessive type VII OI due to a loss-of-function mutation that results in the loss of 3-hydroxylation of the proline residue at position 986 (P986) in both $\alpha 1$ (I) and a1(II) collagen chains[286]. Of these three models, skeletal muscle function has only 
been partially evaluated in the $+/$ G610C and Collal ${ }^{\mathrm{Jt} / \mathrm{+}}$ mouse, with $+/ G 610 \mathrm{C}$ mice exhibiting normal muscle size and function, while $\mathrm{Collal}^{\mathrm{Jrt} / \mathrm{+}}$ mice exhibit reduced muscle size and function. Furthermore, gastrocnemius mitochondrial respiration was equivalent to WT littermates in the +/oim mouse (Figure III-S1), modeling a mild to moderate patient type I/IV OI, that does not exhibit muscle weakness[3]. This further supports the correlation between muscle weakness and mitochondrial function.

Approach: Hindlimb muscle (soleus, gastrocnemius, and tibialis anterior) contractile generating force will be assessed in six male and six female mice by measuring in vivo contractile generating force as previously described[3]. Parameters of mitochondrial function (mitochondrial respiration and citrate synthase activity in isolated mitochondria from gastrocnemius and quadriceps muscles) and mitochondrial content (citrate synthase activity in whole gastrocnemius and quadriceps muscles) will be evaluated in six male and six female mice of the three distinct OI (+/G610C, Collal ${ }^{\mathrm{Srt} /+}$, and $\left.C R T A P^{-/}\right)$mouse models, as well as in their age and sex matched WT littermates. Mitochondria will be isolated from left and right fresh mixed gastrocnemius tissue, and mitochondrial respiration will be evaluated using the Orosboros Oxygraph-2k (Orosboros Instruments; Innsbruck, Austria) via the addition of malate and glutamate (steady-state oxygen flux), titration of ADP (oxygen flux through complex I), titration of succinate (oxygen flux through complex I + II), and addition of FCCP (uncoupled respiration). Finally, cytochrome $\mathrm{C}$ will be added to assess the quality of mitochondrial isolation. Citrate synthase activity will be determined in mixed quadriceps muscle and in mitochondria isolated from mixed gastrocnemius muscle as a measure of mitochondrial content and 
function, respectively. This study will begin to elucidate if there is a severity and/or mutation specific relationship between muscle strength and mitochondrial function in OI.

\section{Significance and impact}

The most prominent manifestation of OI is bone fragility, with clinicians and researchers only recently beginning to consider skeletal muscle weakness as part of the pathophysiology of OI. Historically skeletal muscle weakness in OI has been attributed to inactivity, but recent research has demonstrated inherent muscle weakness in both mouse models and OI patients, including the finding that $80 \%$ of patients with mild OI exhibit muscle force deficits $[3,4,6,47,48]$. The findings presented in this dissertation were aimed to begin elucidation of the mechanisms esponsible for skeletal muscle weakness in OI by characterizing mitochondrial health and function in a mouse modeling a severe human type III OI. These future proposed objectives will lead to a better and more comprehensive understanding of the relationship and mechanisms underlying mitochondrial function and skeletal muscle weakness in OI. Understanding these mechanisms are crucial to identifying novel therapeutic targets addressing muscle weakness in OI, which will ultimately lead to improved bone strength. 


\section{BIBLIOGRAPHY}

[1] J.C. Marini, A. Forlino, H.P. Bächinger, N.J. Bishop, P.H. Byers, A. De Paepe, F. Fassier, N. Fratzl-Zelman, K.M. Kozloff, D. Krakow, K. Montpetit, O. Semler, Osteogenesis imperfecta, Nat. Rev. Dis. Prim. 3 (2017) 17052. https://doi.org/10.1038/nrdp.2017.52.

[2] R. Marom, B.M. Rabenhorst, R. Morello, MANAGEMENT OF ENDOCRINE DISEASE: Osteogenesis imperfecta: an update on clinical features and therapies, Eur. J. Endocrinol. 1 (2020). https://doi.org/10.1530/eje-20-0299.

[3] B.A. Gentry, J.A. Ferreira, A.J. McCambridge, M. Brown, C.L. Phillips, Skeletal muscle weakness in osteogenesis imperfecta mice, Matrix Biol. 29 (2010) 638644. https://doi.org/10.1016/j.matbio.2010.06.006.

[4] L.N. Veilleux, V.B. Darsaklis, K. Montpetit, F.H. Glorieux, F. Rauch, Muscle Function in Osteogenesis Imperfecta Type IV, Calcif Tissue Int. 101 (2017) 362370. https://doi.org/10.1007/s00223-017-0287-y.

[5] L.N. Veilleux, F. Rauch, Muscle-Bone Interactions in Pediatric Bone Diseases, Curr. Osteoporos. Rep. 15 (2017) 425-432. https://doi.org/10.1007/s11914-0170396-6.

[6] L.N. Veilleux, M. Lemay, A. Pouliot-Laforte, M.S. Cheung, F.H. Glorieux, F. Rauch, Muscle anatomy and dynamic muscle function in osteogenesis imperfecta type I, J Clin Endocrinol Metab. 99 (2014) E356-62.

https://doi.org/10.1210/jc.2013-3209. 
[7] G.J.A. Cropp, D.N. Myers, PHYSIOLOGICAL EVIDENCE OF HYPERMETABOLISM IN OSTEOGENESIS IMPERFECTA, Pediatrics. 49 (1972) 375. http://pediatrics.aappublications.org/content/49/3/375.abstract.

[8] I. Boraschi-Diaz, J.T. Tauer, O. El-Rifai, D. Guillemette, G. Lefebvre, F. Rauch, M. Ferron, S. V Komarova, Metabolic phenotype in the mouse model of osteogenesis imperfecta, J Endocrinol. 234 (2017) 279-289. https://doi.org/10.1530/JOE-17-0335.

[9] R. Dalgleish, The human type I collagen mutation database, Oxford University Press, 1997. http://www.le.ac.uk/depts/ge/collagen/collagen.html (accessed October 8, 2020).

[10] R. Dalgleish, The Human Collagen Mutation Database 1998, 1998. http://www.le.ac.uk/genetics/collagen/ (accessed November 30, 2020).

[11] S.H. Ralston, M.S. Gaston, Management of Osteogenesis Imperfecta, Front. Endocrinol. (Lausanne). 10 (2020). https://doi.org/10.3389/fendo.2019.00924.

[12] J. Etich, L. Leßmeier, M. Rehberg, H. Sill, F. Zaucke, C. Netzer, O. Semler, Osteogenesis imperfecta—pathophysiology and therapeutic options, Mol. Cell. Pediatr. 7 (2020). https://doi.org/10.1186/s40348-020-00101-9.

[13] D.O. Sillence, A. Senn, D.M. Danks, Genetic heterogeneity in osteogenesis imperfecta, J. Med. Genet. 16 (1979) 101-116. http://www.ncbi.nlm.nih.gov/pmc/articles/PMC1012733/.

[14] C.L. Phillips, Y. Jeong, Osteogenesis Imperfecta: Muscle-Bone Interactions when 
Bi-directionally Compromised, Curr. Osteoporos. Rep. 16 (2018) 478-489. https://doi.org/10.1007/s11914-018-0456-6.

[15] J. Etich, M. Rehberg, B. Eckes, G. Sengle, O. Semler, F. Zaucke, Signaling pathways affected by mutations causing osteogenesis imperfecta, Cell. Signal. 76 (2020) 109789. https://doi.org/10.1016/j.cellsig.2020.109789.

[16] R. Marom, Y.-C. Lee, I. Grafe, B. Lee, Pharmacological and biological therapeutic strategies for osteogenesis imperfecta, Am. J. Med. Genet. Part C Semin. Med. Genet. 172 (2016) 367-383. https://doi.org/10.1002/ajmg.c.31532.

[17] R. Morello, Osteogenesis imperfecta and therapeutics, Matrix Biol. 71-72 (2018) 294-312. https://doi.org/10.1016/j.matbio.2018.03.010.

[18] J. Harrington, E. Sochett, M.B. Chb, A. Howard, Update on the Evaluation and Treatment of Osteogenesis Imperfecta, (n.d.). https://doi.org/10.1016/j.pcl.2014.08.010.

[19] K. Dwan, C.A. Phillipi, R.D. Steiner, D. Basel, Bisphosphonate therapy for osteogenesis imperfecta, Cochrane Database Syst. Rev. 2016 (2016). https://doi.org/10.1002/14651858.CD005088.pub4.

[20] S. Ma, E.L. Goh, A. Jin, R. Bhattacharya, O.R. Boughton, B. Patel, A. Karunaratne, N.T. Vo, R. Atwood, J.P. Cobb, U. Hansen, R.L. Abel, Long-term effects of bisphosphonate therapy: Perforations, microcracks and mechanical properties, Sci. Rep. 7 (2017) 1-10. https://doi.org/10.1038/srep43399.

[21] C.A. Migliorati, M.A. Siegel, L.S. Elting, Bisphosphonate-associated 
osteonecrosis: a long-term complication of bisphosphonate treatment, Lancet Oncol. 7 (2006) 508-514. https://doi.org/10.1016/S1470-2045(06)70726-4.

[22] J.T. Tauer, S. Abdullah, F. Rauch, Effect of Anti-TGF- $\beta$ Treatment in a Mouse Model of Severe Osteogenesis Imperfecta, J. Bone Miner. Res. 34 (2019) $207-$ 214. https://doi.org/10.1002/jbmr.3617.

[23] D. Gatti, M. Rossini, O. Viapiana, M.R. Povino, S. Liuzza, E. Fracassi, L. Idolazzi, S. Adami, Teriparatide treatment in adult patients with osteogenesis imperfecta type i, Calcif. Tissue Int. 93 (2013) 448-452. https://doi.org/10.1007/s00223-0139770-2.

[24] M. Cardinal, A. Dessain, T. Roels, S. Lafont, M.S. Ominsky, J.P. Devogelaer, D. Chappard, G. Mabilleau, P. Ammann, C. Nyssen-Behets, D.H. Manicourt, Sclerostin-Antibody Treatment Decreases Fracture Rates in Axial Skeleton and Improves the Skeletal Phenotype in Growing oim/oim Mice, Calcif. Tissue Int. 106 (2020) 494-508. https://doi.org/10.1007/s00223-019-00655-5.

[25] S. Ricard-Blum, The Collagen Family, Cold Spring Harb. Perspect. Biol. 3 (2011) 1-19. https://doi.org/10.1101/cshperspect.a004978.

[26] S. Viguet-Carrin, P. Garnero, P.D. Delmas, The role of collagen in bone strength, Osteoporos. Int. 17 (2006) 319-336. https://doi.org/10.1007/s00198-005-2035-9.

[27] B. Brodsky, N.K. Shah, Protein motifs. 8. The triple-helix motif in proteins., FASEB J. 9 (1995) 1537-1546. https://doi.org/10.1096/fasebj.9.15.8529832.

[28] A.L. Fidler, S.P. Boudko, A. Rokas, B.G. Hudson, The triple helix of collagens-an 
ancient protein structure that enabled animal multicellularity and tissue evolution, (2018). https://doi.org/10.1242/jcs.203950.

[29] A. Sorushanova, L.M. Delgado, Z. Wu, N. Shologu, A. Kshirsagar, R. Raghunath, A.M. Mullen, Y. Bayon, A. Pandit, M. Raghunath, D.I. Zeugolis, The Collagen Suprafamily: From Biosynthesis to Advanced Biomaterial Development, Adv. Mater. 31 (2019). https://doi.org/10.1002/adma.201801651.

[30] M. Wu, J.S. Crane, Biochemistry, Collagen Synthesis, StatPearls Publishing, 2018. http://www.ncbi.nlm.nih.gov/pubmed/29939531 (accessed October 8, 2020).

[31] H. Lodish, A. Berk, S.L. Zipursky, P. Matsudaira, D. Baltimore, J. Darnell, Collagen: The Fibrous Proteins of the Matrix, (2000). https://www.ncbi.nlm.nih.gov/books/NBK21582/ (accessed October 8, 2020).

[32] E.G. Canty, K.E. Kadler, Procollagen trafficking, processing and fibrillogenesis, J. Cell Sci. 118 (2005) 1341-1353. https://doi.org/10.1242/jcs.01731.

[33] E. Makareeva, N.A. Aviles, S. Leikin, Chaperoning osteogenesis: New proteinfolding disease paradigms, Trends Cell Biol. 21 (2011) 168-176. https://doi.org/10.1016/j.tcb.2010.11.007.

[34] H. Lodish, A. Berk, S.L. Zipursky, P. Matsudaira, D. Baltimore, J. Darnell, Muscle: A Specialized Contractile Machine, (2000). https://www.ncbi.nlm.nih.gov/books/NBK21670/ (accessed September 3, 2020).

[35] X. Wang, R.A. Bank, J.M. Tekoppele, C.M. Agrawal, The role of collagen in determining bone mechanical properties, J. Orthop. Res. 19 (2001) 1021-1026. 
https://doi.org/10.1016/S0736-0266(01)00047-X.

[36] B. Grabner, W.J. Landis, P. Roschger, S. Rinnerthaler, H. Peterlik, K. Klaushofer, P. Fratzl, Age- and genotype-dependence of bone material properties in the osteogenesis imperfecta murine model (oim), Bone. 29 (2001) 453-457. https://doi.org/10.1016/S8756-3282(01)00594-4.

[37] R.F. Pereira, E.L. Hume, K.W. Halford, D.J. Prockop, Bone fragility in transgenic mice expressing a mutated gene for type I procollagen (COL1A1) parallels the age-dependent phenotype of human osteogenesis imperfecta, J. Bone Miner. Res. 10 (2009) 1837-1843. https://doi.org/10.1002/jbmr.5650101202.

[38] H. Leng, X.N. Dong, X. Wang, Progressive post-yield behavior of human cortical bone in compression for middle-aged and elderly groups, J. Biomech. 42 (2009) 491-497. https://doi.org/10.1016/j.jbiomech.2008.11.016.

[39] S.D. Chipman, H.O. Sweet, D.J. McBride, M.T. Davisson, S.C. Marks, A.R. Shuldiner, R.J. Wenstrup, D.W. Rowe, J.R. Shapiro, Defective pro alpha 2(I) collagen synthesis in a recessive mutation in mice: a model of human osteogenesis imperfecta, Proc. Natl. Acad. Sci. U. S. A. 90 (1993) 1701-1705. http://www.ncbi.nlm.nih.gov/pmc/articles/PMC45947/.

[40] F.M. Pope, A.C. Nicholls, J. McPheat, P. Talmud, R. Owen, Collagen genes and proteins in osteogenesis imperfecta, J. Med. Genet. 22 (1985) 466-478. https://doi.org/10.1136/jmg.22.6.466.

[41] T. Pihlajaniemi, L.A. Dickson, F.M. Pope, V.R. Korhonen, A. Nicholls, D.J. 
Prockop, J.C. Myers, Osteogenesis imperfecta: Cloning of a pro- $\alpha 2$ (I) collagen gene with a frameshift mutation, J. Biol. Chem. 259 (1984) 12941-12944.

[42] K. Misof, $\div$ W J Landis, K. Klaushofer, P. Fratzl, Collagen from the Osteogenesis Imperfecta Mouse Model (oim) Shows Reduced Resistance Against Tensile Stress, 1997.

[43] P. Fratzl, $\ddagger$ O Paris, K. Klaushofer, W.J. Landis, Bone Mineralization in an Osteogenesis Imperfecta Mouse Model Studied by Small-Angle X-ray Scattering, 1996.

[44] E. Miller, D. Delos, T. Baldini, T.M. Wright, N. Pleshko Camacho, Abnormal mineral-matrix interactions are a significant contributor to fragility in oim/oim bone, Calcif. Tissue Int. 81 (2007) 206-214. https://doi.org/10.1007/s00223-0079045-x.

[45] N.P. Camacho, L. Hou, T.R. Toledano, W. Alex Ilg, C.F. Brayton, C.L. Raggio, L. Root, A.L. Boskey, The material basis for reduced mechanical properties in oim mice bones, J. Bone Miner. Res. 14 (1999) 264-272. https://doi.org/10.1359/jbmr.1999.14.2.264.

[46] T. Takken, H.C. Terlingen, P.J. Helders, H. Pruijs, C.K. Van der Ent, R.H. Engelbert, Cardiopulmonary fitness and muscle strength in patients with osteogenesis imperfecta type I, J Pediatr. 145 (2004) 813-818. https://doi.org/10.1016/j.jpeds.2004.08.003.

[47] A. Pouliot-Laforte, L.N. Veilleux, F. Rauch, M. Lemay, Physical activity in youth 
with osteogenesis imperfecta type I, J. Musculoskelet. Neuronal Interact. 15 (2015) 171-176. /pmc/articles/PMC5133720/?report=abstract (accessed October 9, 2020).

[48] L.N. Veilleux, P. Trejo, F. Rauch, Muscle abnormalities in osteogenesis imperfecta, J. Musculoskelet. Neuronal Interact. 17 (2017) 1-7. http://www.ncbi.nlm.nih.gov/pmc/articles/PMC5492314/.

[49] Y. Jeong, S.M. Carleton, B.A. Gentry, X. Yao, J.A. Ferreira, D.J. Salamango, M. Weis, A.K. Oestreich, A.M. Williams, M.G. McCray, D.R. Eyre, M. Brown, Y. Wang, C.L. Phillips, Hindlimb Skeletal Muscle Function and Skeletal Quality and Strength in +/G610C Mice With and Without Weight-Bearing Exercise, J Bone Min. Res. 30 (2015) 1874-1886. https://doi.org/10.1002/jbmr.2518.

[50] E. Daley, E.A. Streeten, J.D. Sorkin, N. Kuznetsova, S.A. Shapses, S.M. Carleton, A.R. Shuldiner, J.C. Marini, C.L. Phillips, S.A. Goldstein, S. Leikin, D.J. McBride Jr., Variable bone fragility associated with an Amish COL1A2 variant and a knock-in mouse model, J. Bone Miner. Res. 25 (2010) 247-261. https://doi.org/10.1359/jbmr.090720.

[51] F. Chen, R. Guo, S. Itoh, L. Moreno, E. Rosenthal, T. Zappitelli, R.A. Zirngibl, A. Flenniken, W. Cole, M. Grynpas, L.R. Osborne, W. Vogel, L. Adamson, J. Rossant, J.E. Aubin, First Mouse Model for Combined Osteogenesis Imperfecta and Ehlers-Danlos Syndrome, J. Bone Miner. Res. 29 (2014) 1412-1423. https://doi.org/10.1002/jbmr.2177.

[52] D.M. Abdelaziz, S. Abdullah, C. Magnussen, A. Ribeiro-da-Silva, S. V Komarova, F. Rauch, L.S. Stone, Behavioral signs of pain and functional impairment in a 
mouse model of osteogenesis imperfecta, Bone. 81 (2015) 400-406.

https://doi.org/https://doi.org/10.1016/j.bone.2015.08.001.

[53] J.M. Bowen, G.J. Sobey, N.P. Burrows, M. Colombi, M.E. Lavallee, F. Malfait, C.A. Francomano, Ehlers-Danlos syndrome, classical type, Am. J. Med. Genet. Part C Semin. Med. Genet. 175 (2017) 27-39. https://doi.org/10.1002/ajmg.c.31548.

[54] T. Miklovic, V.C. Sieg, Ehlers Danlos Syndrome, StatPearls Publishing, 2020. http://www.ncbi.nlm.nih.gov/pubmed/31747221 (accessed November 30, 2020).

[55] F. Zurlo, K. Larson, C. Bogardus, E. Ravussin, Skeletal muscle metabolism is a major determinant of resting energy expenditure, J. Clin. Invest. 86 (1990) $1423-$ 1427. https://doi.org/10.1172/JCI114857.

[56] S.B. Heymsfield, D.M. Thomas, A. Bosy-Westphal, M.J. Müller, The anatomy of resting energy expenditure: body composition mechanisms, Eur. J. Clin. Nutr. 73 (2019) 166-171. https://doi.org/10.1038/s41430-018-0319-3.

[57] H.M. Frost, Bone's mechanostat: A 2003 update, Anat. Rec. Part A Discov. Mol. Cell. Evol. Biol. 275A (2003) 1081-1101. https://doi.org/10.1002/ar.a.10119.

[58] R.L. Duncan, C.H. Turner, Mechanotransduction and the functional response of bone to mechanical strain, Calcif. Tissue Int. 57 (1995) 344-358. https://doi.org/10.1007/BF00302070.

[59] J. Isaacson, M. Brotto, Physiology of Mechanotransduction: How Do Muscle and Bone "Talk" to One Another?, Clin. Rev. Bone Miner. Metab. 12 (2014) 77-85. 
https://doi.org/10.1007/s12018-013-9152-3.

[60] M. Brotto, M.L. Johnson, Endocrine crosstalk between muscle and bone, Curr. Osteoporos. Rep. 12 (2014) 135-141. https://doi.org/10.1007/s11914-014-0209-0.

[61] M. Brotto, L. Bonewald, Bone and muscle: Interactions beyond mechanical, Bone. 80 (2015) 109-114. https://doi.org/10.1016/j.bone.2015.02.010.

[62] M.F. Young, Bone matrix proteins: their function, regulation, and relationship to osteoporosis., Osteoporos. Int. 14 Suppl 3 (2003). https://doi.org/10.1007/s00198002-1342-7.

[63] R. Florencio-Silva, G.R.D.S. Sasso, E. Sasso-Cerri, M.J. Simões, P.S. Cerri, Biology of Bone Tissue: Structure, Function, and Factors That Influence Bone Cells, Biomed Res. Int. 2015 (2015). https://doi.org/10.1155/2015/421746.

[64] H.K. Datta, W.F. Ng, J.A. Walker, S.P. Tuck, S.S. Varanasi, The cell biology of bone metabolism, J. Clin. Pathol. 61 (2008) 577-587. https://doi.org/10.1136/jcp.2007.048868.

[65] W.J. Boyle, W.S. Simonet, D.L. Lacey, Osteoclast differentiation and activation, Nature. 423 (2003) 337-342. https://doi.org/10.1038/nature01658.

[66] S.L. Teitelbaum, Bone resorption by osteoclasts, Science (80-. ). 289 (2000) 15041508. https://doi.org/10.1126/science.289.5484.1504.

[67] N.A. Sims, J.H. Gooi, Bone remodeling: Multiple cellular interactions required for coupling of bone formation and resorption, Semin. Cell Dev. Biol. 19 (2008) 444451. https://doi.org/10.1016/j.semcdb.2008.07.016. 
[68] P. Katsimbri, The biology of normal bone remodelling, Eur. J. Cancer Care (Engl). 26 (2017) e12740. https://doi.org/10.1111/ecc.12740.

[69] L.F. Bonewald, The amazing osteocyte, J. Bone Miner. Res. 26 (2011) 229-238. https://doi.org/10.1002/jbmr.320.

[70] J.L. Pathak, N. Bravenboer, J. Klein-Nulend, The Osteocyte as the New Discovery of Therapeutic Options in Rare Bone Diseases, Front. Endocrinol. (Lausanne). 11 (2020) 405. https://doi.org/10.3389/fendo.2020.00405.

[71] D.A. Rivas, R.A. Fielding, Skeletal Muscle, in: Encycl. Hum. Nutr., Elsevier Inc., 2012: pp. 193-199. https://doi.org/10.1016/B978-0-12-375083-9.00188-4.

[72] B.K. Pedersen, Muscle as a Secretory Organ, in: Compr. Physiol., John Wiley \& Sons, Inc., Hoboken, NJ, USA, 2013: pp. 1337-1362. https://doi.org/10.1002/cphy.c120033.

[73] W.R. Frontera, J. Ochala, Skeletal Muscle: A Brief Review of Structure and Function, Behav. Genet. 45 (2015) 183-195. https://doi.org/10.1007/s00223-0149915-y.

[74] J. Chal, O. Pourquié, Making muscle: Skeletal myogenesis in vivo and in vitro, Dev. 144 (2017) 2104-2122. https://doi.org/10.1242/dev.151035.

[75] M. Buckingham, L. Bajard, T. Chang, P. Daubas, J. Hadchouel, S. Meilhac, D. Montarras, D. Rocancourt, F. Relaix, The formation of skeletal muscle: From somite to limb, J. Anat. 202 (2003) 59-68. https://doi.org/10.1046/j.14697580.2003.00139.x. 
[76] P.H. Francis-West, L. Antoni, K. Anakwe, Regulation of myogenic differentiation in the developing limb bud, J. Anat. 202 (2003) 69-81. https://doi.org/10.1046/j.1469-7580.2003.00136.x.

[77] M. Buckingham, L. Bajard, T. Chang, P. Daubas, J. Hadchouel, S. Meilhac, D. Montarras, D. Rocancourt, F. Relaix, The formation of skeletal muscle: From somite to limb, J. Anat. 202 (2003) 59-68. https://doi.org/10.1046/j.14697580.2003.00139.x.

[78] S. Schiaffino, C. Reggiani, Fiber Types in Mammalian Skeletal Muscles, Physiol. Rev. 91 (2011) 1447-1531. https://doi.org/10.1152/physrev.00031.2010.

[79] J. Talbot, L. Maves, Skeletal muscle fiber type: using insights from muscle developmental biology to dissect targets for susceptibility and resistance to muscle disease, Wiley Interdiscip. Rev. Dev. Biol. 5 (2016) 518-534. https://doi.org/10.1002/wdev.230.

[80] M. Picard, R.T. Hepple, Y. Burelle, Mitochondrial functional specialization in glycolytic and oxidative muscle fibers: tailoring the organelle for optimal function, Am. J. Physiol. Physiol. 302 (2011) C629-C641. https://doi.org/10.1152/ajpcell.00368.2011.

[81] H. Yin, F. Price, M.A. Rudnicki, Satellite cells and the muscle stem cell niche, Physiol. Rev. 93 (2013) 23-67. https://doi.org/10.1152/physrev.00043.2011.

[82] J.E. Morgan, T.A. Partridge, Muscle satellite cells, Int. J. Biochem. Cell Biol. 35 (2003) 1151-1156. https://doi.org/10.1016/S1357-2725(03)00042-6. 
[83] A. MAURO, Satellite cell of skeletal muscle fibers., J. Biophys. Biochem. Cytol. 9 (1961) 493-495. https://doi.org/10.1083/jcb.9.2.493.

[84] P.S. Zammit, Function of the myogenic regulatory factors Myf5, MyoD, Myogenin and MRF4 in skeletal muscle, satellite cells and regenerative myogenesis, Semin. Cell Dev. Biol. 72 (2017) 19-32. https://doi.org/10.1016/j.semcdb.2017.11.011.

[85] C.A. Collins, I. Olsen, P.S. Zammit, L. Heslop, A. Petrie, T.A. Partridge, J.E. Morgan, Stem cell function, self-renewal, and behavioral heterogeneity of cells from the adult muscle satellite cell niche, Cell. 122 (2005) 289-301. https://doi.org/10.1016/j.cell.2005.05.010.

[86] BISCHOFF, R., The satellite cell and muscle regeneration, Myology. (1994). https://ci.nii.ac.jp/naid/10018625843 (accessed October 27, 2020).

[87] H.L. Sweeney, D.W. Hammers, Muscle contraction, Cold Spring Harb. Perspect. Biol. 10 (2018). https://doi.org/10.1101/cshperspect.a023200.

[88] B.D. Hugh Huxley, D. Jean Hanson, Changes in the Cross-Striations of Muscle during Contraction and Stretch and their Structural Interpretation, 1880.

[89] S. Lange, N. Pinotsis, I. Agarkova, E. Ehler, The M-band: The underestimated part of the sarcomere, Biochim. Biophys. Acta - Mol. Cell Res. 1867 (2020) 118440. https://doi.org/10.1016/j.bbamcr.2019.02.003.

[90] P.K. Luther, J.M. Squire, The intriguing dual lattices of the myosin filaments in vertebrate striated muscles: Evolution and advantage, Biology (Basel). 3 (2014) 
846-865. https://doi.org/10.3390/biology3040846.

[91] C.A. Henderson, C.G. Gomez, S.M. Novak, L. Mi-Mi, C.C. Gregorio, Overview of the Muscle Cytoskeleton, in: Compr. Physiol., John Wiley \& Sons, Inc., Hoboken, NJ, USA, 2017: pp. 891-944. https://doi.org/10.1002/cphy.c160033.

[92] A.R. Gillies, R.L. Lieber, Structure and function of the skeletal muscle extracellular matrix, Muscle Nerve. 44 (2011) 318-331[1] A.R. Gillies, R.L. Lieber, Structure an. https://doi.org/10.1002/mus.22094.

[93] C. McCuller, A.L. Callahan, Physiology, Skeletal Muscle, StatPearls Publishing, 2019. http://www.ncbi.nlm.nih.gov/pubmed/30725824 (accessed September 2, 2020).

[94] T.J. Patel, R.L. Lieber, 12 force transmission in skeletal muscle, Exerc. Sport Sci. Rev. 25 (1997) 321-364. https://doi.org/10.1249/00003677-199700250-00014.

[95] J.R. Bendall, The elastin content of various muscles of beef animals, J. Sci. Food Agric. 18 (1967) 553-558. https://doi.org/10.1002/jsfa.2740181201.

[96] E. Dransfield, Intramuscular composition and texture of beef muscles, J. Sci. Food Agric. 28 (1977) 833-842. https://doi.org/10.1002/jsfa.2740280910.

[97] L.M. Biga, S. Dawson, A. Harwell, R. Hopkins, J. Kaufmann, M. LeMaster, P. Matern, K. Morrison-Graham, D. Quick, J. Runyeon, 10.2 Skeletal Muscle, (n.d.).

[98] A.F. Huxley, D.R. Niedergerke, STRUCTURAL CHANGES IN MUSCLE DURING CONTRACTION Interference Microscopy of Living Muscle Fibres, 1954. 
[99] R.H. Fitts, HIGHLIGHTED TOPIC Fatigue Mechanisms Determining Exercise Performance The cross-bridge cycle and skeletal muscle fatigue, J Appl Physiol. 104 (2008) 551-558. https://doi.org/10.1152/japplphysiol.01200.2007.-The.

[100] M. Irving, Regulation of Contraction by the Thick Filaments in Skeletal Muscle, Biophys. J. 113 (2017) 2579-2594. https://doi.org/10.1016/j.bpj.2017.09.037.

[101] J. Walklate, Z. Ujfalusi, M.A. Geeves, Myosin isoforms and the mechanochemical cross-bridge cycle, J. Exp. Biol. 219 (2016) 168-174. https://doi.org/10.1242/jeb.124594.

[102] J. Squire, Special issue: The actin-myosin interaction in muscle: Background and overview, Int. J. Mol. Sci. 20 (2019). https://doi.org/10.3390/ijms20225715.

[103] L. Bonewald, Use it or lose it to age: A review of bone and muscle communication, Bone. 120 (2019) 212-218. https://doi.org/10.1016/j.bone.2018.11.002.

[104] F. Doyle, J. Brown, C. Lachance, RELATION BETWEEN BONE MASS AND MUSCLE WEIGHT, Lancet. 295 (1970) 391-393. https://doi.org/10.1016/S01406736(70)91520-5.

[105] H.M. Frost, Wolff's Law and bone's structural adaptations to mechanical usage: an overview for clinicians., Angle Orthod. 64 (1994) 175-188. https://doi.org/10.1043/0003-3219(1994)064<0175:WLABSA>2.0.CO;2.

[106] F. HM, A 2003 update of bone physiology and Wolff's Law for clinicians, Angle Orthod. 74 (2004). https://doi.org/10.1043/0003- 
3219(2004)074<0003:AUOBPA>2.0.CO;2.

[107] H.M. Frost, Perspectives: A proposed general model of the "Mechanostat" (Suggestions from a new skeletal-biologic paradigm), Anat. Rec. 244 (1996) 139147. https://doi.org/10.1002/(SICI)1097-0185(199602)244:2<139::AIDAR1>3.0.CO;2-X.

[108] J. Zhou, J. Yi, L. Bonewald, Muscle-Bone Crosstalk in Amyotrophic Lateral Sclerosis, Curr. Osteoporos. Rep. 13 (2015) 274-279. https://doi.org/10.1007/s11914-015-0281-0.

[109] A. Boulanger Piette, D. Hamoudi, L. Marcadet, F. Morin, A. Argaw, L. Ward, J. Frenette, Targeting the Muscle-Bone Unit: Filling Two Needs with One Deed in the Treatment of Duchenne Muscular Dystrophy, Curr. Osteoporos. Rep. 16 (2018) 541-553. https://doi.org/10.1007/s11914-018-0468-2.

[110] D. Grimm, J. Grosse, M. Wehland, V. Mann, J.E. Reseland, A. Sundaresan, T.J. Corydon, The impact of microgravity on bone in humans, Bone. 87 (2016) 44-56. https://doi.org/10.1016/j.bone.2015.12.057.

[111] T. Bettis, B.J. Kim, M.W. Hamrick, Impact of muscle atrophy on bone metabolism and bone strength: implications for muscle-bone crosstalk with aging and disuse, Osteoporos. Int. 29 (2018) 1713-1720. https://doi.org/10.1007/s00198-018-45701.

[112] A.R. Hargens, L. Vico, Long-duration bed rest as an analog to microgravity, J. Appl. Physiol. 120 (2016) 891-903. 
https://doi.org/10.1152/japplphysiol.00935.2015.

[113] E.K. Paluch, C.M. Nelson, N. Biais, B. Fabry, J. Moeller, B.L. Pruitt, C. Wollnik, G. Kudryasheva, F. Rehfeldt, W. Federle, Mechanotransduction: Use the force(s), BMC Biol. 13 (2015). https://doi.org/10.1186/s12915-015-0150-4.

[114] S.L. Dallas, M. Prideaux, L.F. Bonewald, The osteocyte: An endocrine cell . . . and more, Endocr. Rev. 34 (2013) 658-690. https://doi.org/10.1210/er.2012-1026.

[115] M. Van Brussel, T. Takken, C.S. Uiterwaal, H.J. Pruijs, J. Van der Net, P.J. Helders, R.H. Engelbert, Physical training in children with osteogenesis imperfecta, J Pediatr. 152 (2008) 111-6, 116 e1. https://doi.org/10.1016/j.jpeds.2007.06.029.

[116] F.G.F. Tresguerres, J. Torres, J. López-Quiles, G. Hernández, J.A. Vega, I.F. Tresguerres, The osteocyte: A multifunctional cell within the bone, Ann. Anat. 227 (2020) 151422. https://doi.org/10.1016/j.aanat.2019.151422.

[117] K. Scheld, A. Zittermann, M. Heer, B. Herzog, C. Mika, C. Drummer, P. Stehle, Nitrogen metabolism and bone metabolism markers in healthy adults during 16 weeks of bed rest, Clin. Chem. 47 (2001) 1688-1695. https://doi.org/10.1093/clinchem/47.9.1688.

[118] T. Lang, A. LeBlanc, H. Evans, Y. Lu, H. Genant, A. Yu, Cortical and trabecular bone mineral loss from the spine and hip in long-duration spaceflight, J. Bone Miner. Res. 19 (2004) 1006-1012. https://doi.org/10.1359/JBMR.040307.

[119] S.A. Novotny, G.L. Warren, A.S. Lin, R.E. Guldberg, K.A. Baltgalvis, D.A. Lowe, 
Bone is functionally impaired in dystrophic mice but less so than skeletal muscle, Neuromuscul. Disord. 21 (2011) 183-193. https://doi.org/10.1016/j.nmd.2010.12.002.

[120] C. Tagliaferri, Y. Wittrant, M.J. Davicco, S. Walrand, V. Coxam, Muscle and bone, two interconnected tissues, Ageing Res. Rev. 21 (2015) 55-70. https://doi.org/10.1016/j.arr.2015.03.002.

[121] Y. Yuan, X. Chen, L. Zhang, J. Wu, J. Guo, D. Zou, B. Chen, Z. Sun, C. Shen, J. Zou, The roles of exercise in bone remodeling and in prevention and treatment of osteoporosis, Prog. Biophys. Mol. Biol. 122 (2016) 122-130. https://doi.org/10.1016/j.pbiomolbio.2015.11.005.

[122] X. Tu, J. Delgado-Calle, K.W. Condon, M. Maycas, H. Zhang, N. Carlesso, M.M. Taketo, D.B. Burr, L.I. Plotkin, T. Bellido, Osteocytes mediate the anabolic actions of canonical Wnt//-catenin signaling in bone, Proc. Natl. Acad. Sci. U. S. A. 112 (2015) E478-E486. https://doi.org/10.1073/pnas.1409857112.

[123] D.A. Glass, G. Karsenty, Molecular Bases of the Regulation of Bone Remodeling by the Canonical Wnt Signaling Pathway, Curr. Top. Dev. Biol. 73 (2006) 43-84. https://doi.org/10.1016/S0070-2153(05)73002-7.

[124] P. Duan, L.F. Bonewald, The role of the wnt/ $\beta$-catenin signaling pathway in formation and maintenance of bone and teeth, Int. J. Biochem. Cell Biol. 77 (2016) 23-29. https://doi.org/10.1016/j.biocel.2016.05.015.

[125] K.L. Troy, M.E. Mancuso, T.A. Butler, J.E. Johnson, Exercise early and often: 
Effects of physical activity and exercise on women's bone health, Int. J. Environ. Res. Public Health. 15 (2018). https://doi.org/10.3390/ijerph15050878.

[126] C.M. Weaver, C.M. Gordon, K.F. Janz, H.J. Kalkwarf, J.M. Lappe, R. Lewis, M. O'Karma, T.C. Wallace, B.S. Zemel, The National Osteoporosis Foundation's position statement on peak bone mass development and lifestyle factors: a systematic review and implementation recommendations, Osteoporos. Int. 27 (2016) 1281-1386. https://doi.org/10.1007/s00198-015-3440-3.

[127] T. Takken, H.C. Terlingen, P.J.M. Helders, H. Pruijs, C.K. Van Der Ent, R.H.H. Engelbert, CARDIOPULMONARY FITNESS AND MUSCLE STRENGTH IN PATIENTS WITH OSTEOGENESIS IMPERFECTA TYPE I, (n.d.). https://doi.org/10.1016/j.jpeds.2004.08.003.

[128] R.H. Engelbert, C.S. Uiterwaal, W.J. Gerver, J.J. Van Der Net, H.E. Pruijs, P.J. Helders, Osteogenesis imperfecta in childhood: Impairment and disability. A prospective study with 4-year follow-up, Arch. Phys. Med. Rehabil. 85 (2004) 772-778. https://doi.org/10.1016/j.apmr.2003.08.085.

[129] H. Hoyer-Kuhn, O. Semler, C. Stark, N. Struebing, O. Goebel, E. Schoenau, A specialized rehabilitation approach improves mobility in children with osteogenesis imperfecta, J. Musculoskelet. Neuronal Interact. 14 (2014) 445-453. http://europepmc.org/abstract/MED/25524970.

[130] M. Vanleene, S.J. Shefelbine, Therapeutic impact of low amplitude high frequency whole body vibrations on the osteogenesis imperfecta mouse bone, Bone. 53 (2013) 507-514. https://doi.org/10.1016/j.bone.2013.01.023. 
[131] O. Semler, O. Fricke, K. Vezyroglou, C. Stark, A. Stabrey, E. Schoenau, Results of a prospective pilot trial on mobility after whole body vibration in children and adolescents with osteogenesis imperfecta, Clin. Rehabil. 22 (2008) 387-394. https://doi.org/10.1177/0269215507080763.

[132] W. Högler, J. Scott, N. Bishop, P. Arundel, P. Nightingale, M.Z. Mughal, R. Padidela, N. Shaw, N. Crabtree, The Effect of Whole Body Vibration Training on Bone and Muscle Function in Children With Osteogenesis Imperfecta, J. Clin. Endocrinol. Metab. 102 (2017) 2734-2743. https://doi.org/10.1210/jc.2017-00275.

[133] B. Kirk, J. Feehan, G. Lombardi, G. Duque, Muscle, Bone, and Fat Crosstalk: the Biological Role of Myokines, Osteokines, and Adipokines, Curr. Osteoporos. Rep. 18 (2020) 388-400. https://doi.org/10.1007/s11914-020-00599-y.

[134] R.R. Richards, J.L. Mahoney, T. Minas, Influence of soft tissue coverage on the healing of cortical defects in canine diaphyseal bone, Ann. Plast. Surg. 16 (1986) 296-304. https://doi.org/10.1097/00000637-198604000-00005.

[135] L.E. Harry, A. Sandison, E.M. Paleolog, U. Hansen, M.F. Pearse, J. Nanchahal, Comparison of the healing of open tibial fractures covered with either muscle or fasciocutaneous tissue in a murine model, J. Orthop. Res. 26 (2008) 1238-1244. https://doi.org/10.1002/jor.20649.

[136] N. Lara-Castillo, M.L. Johnson, Bone-Muscle Mutual Interactions, Curr. Osteoporos. Rep. 18 (2020) 408-421. https://doi.org/10.1007/s11914-020-006026. 
[137] B.K. Pedersen, A. Steensberg, C. Fischer, C. Keller, P. Keller, P. Plomgaard, M. Febbraio, B. Saltin, Searching for the exercise factor: Is IL-6 a candidate?, in: J. Muscle Res. Cell Motil., Springer, 2003: pp. 113-119. https://doi.org/10.1023/A:1026070911202.

[138] A.C. McPherron, A.M. Lawler, S.-J. Lee, Regulation of skeletal muscle mass in mice by a new TGF-p superfamily member, Nature. 387 (1997) 83. https://doi.org/10.1038/387083a0.

[139] M. Whitham, M.A. Febbraio, The ever-expanding myokinome: Discovery challenges and therapeutic implications, Nat. Rev. Drug Discov. 15 (2016) 719 729. https://doi.org/10.1038/nrd.2016.153.

[140] R. Piccirillo, Exercise-induced myokines with therapeutic potential for muscle wasting, Front. Physiol. 10 (2019) 287. https://doi.org/10.3389/fphys.2019.00287.

[141] Q. Wang, A.C. McPherron, Myostatin inhibition induces muscle fibre hypertrophy prior to satellite cell activation, J. Physiol. 590 (2012) 2151-2165. https://doi.org/10.1113/jphysiol.2011.226001.

[142] Y. Qin, Y. Peng, W. Zhao, J. Pan, H. Ksiezak-Reding, C. Cardozo, Y. Wu, P.D. Pajevic, L.F. Bonewald, W.A. Bauman, W. Qin, Myostatin inhibits osteoblastic differentiation by suppressing osteocyte-derived exosomal microRNA-218: A novel mechanism in muscle-bone communication, J. Biol. Chem. 292 (2017) 11021-11033. https://doi.org/10.1074/jbc.M116.770941.

[143] B. Dankbar, M. Fennen, D. Brunert, S. Hayer, S. Frank, C. Wehmeyer, D. 
Beckmann, P. Paruzel, J. Bertrand, K. Redlich, C. Koers-Wunrau, A. Stratis, A. Korb-Pap, T. Pap, Myostatin is a direct regulator of osteoclast differentiation and its inhibition reduces inflammatory joint destruction in mice, Nat. Med. 21 (2015) 1085.

https://doi.org/10.1038/nm.3917https://www.nature.com/articles/nm.3917\#supple mentary-information.

[144] N. Hiscock, M.H.S. Chan, T. Bisucci, I.A. Darby, M.A. Febbraio, Skeletal myocytes are a source of interleukin- 6 mRNA expression and protein release during contraction: evidence of fiber type specificity, FASEB J. 18 (2004) 992994. https://doi.org/10.1096/fj.03-1259fje.

[145] P. Juffer, R.T. Jaspers, J. Klein-Nulend, A.D. Bakker, Mechanically loaded myotubes affect osteoclast formation, Calcif. Tissue Int. 94 (2014) 319-326. https://doi.org/10.1007/s00223-013-9813-8.

[146] T. Klangjareonchai, H. Nimitphong, S. Saetung, N. Bhirommuang, R. Samittarucksa, S. Chanprasertyothin, R. Sudatip, B. Ongphiphadhanakul, Circulating Sclerostin and Irisin Are Related and Interact with Gender to Influence Adiposity in Adults with Prediabetes, Int. J. Endocrinol. 2014 (2014). https://doi.org/10.1155/2014/261545.

[147] V. Singhal, E.A. Lawson, K.E. Ackerman, P.K. Fazeli, H. Clarke, H. Lee, K. Eddy, D.A. Marengi, N.P. Derrico, M.L. Bouxsein, M. Misra, Irisin levels are lower in young amenorrheic athletes compared with eumenorrheic athletes and non-athletes and are associated with bone density and strength estimates, PLoS 
One. 9 (2014). https://doi.org/10.1371/journal.pone.0100218.

[148] G. Colaianni, C. Cuscito, T. Mongelli, P. Pignataro, C. Buccoliero, P. Liu, P. Lu, L. Sartini, M.D. Comite, G. Mori, A.D. Benedetto, G. Brunetti, T. Yuen, L. Sun, J.E. Reseland, S. Colucci, M.I. New, M. Zaidi, S. Cinti, M. Grano, The myokine irisin increases cortical bone mass, Proc. Natl. Acad. Sci. U. S. A. 112 (2015) 12157-12162. https://doi.org/10.1073/pnas.1516622112.

[149] Y. Kitase, J.A. Vallejo, W. Gutheil, H. Vemula, K. Jähn, J. Yi, J. Zhou, M. Brotto, L.F. Bonewald, $\beta$-aminoisobutyric Acid, 1-BAIBA, Is a Muscle-Derived Osteocyte Survival Factor, Cell Rep. 22 (2018) 1531-1544. https://doi.org/10.1016/j.celrep.2018.01.041.

[150] L.D. Roberts, P. Boström, J.F. O’Sullivan, R.T. Schinzel, G.D. Lewis, A. Dejam, Y.K. Lee, M.J. Palma, S. Calhoun, A. Georgiadi, M.H. Chen, V.S. Ramachandran, M.G. Larson, C. Bouchard, T. Rankinen, A.L. Souza, C.B. Clish, T.J. Wang, J.L. Estall, A.A. Soukas, C.A. Cowan, B.M. Spiegelman, R.E. Gerszten, $\beta$ Aminoisobutyric acid induces browning of white fat and hepatic $\beta$-oxidation and is inversely correlated with cardiometabolic risk factors, Cell Metab. 19 (2014) 96108. https://doi.org/10.1016/j.cmet.2013.12.003.

[151] P. Ducy, C. Desbois, B. Boyce, G. Pinero, B. Story, C. Dunstan, E. Smith, J. Bonadio, S. Goldstein, C. Gundberg, A. Bradley, G. Karsenty, Increased bone formation in osteocalcin-deficient mice, Nature. 382 (1996) 448-452. https://doi.org/10.1038/382448a0.

[152] G. Karsenty, P. Mera, Molecular bases of the crosstalk between bone and muscle, 
Bone. 115 (2018) 43-49. https://doi.org/10.1016/j.bone.2017.04.006.

[153] P. Mera, K. Laue, M. Ferron, C. Confavreux, J. Wei, M. Galán-Díez, A. Lacampagne, S.J. Mitchell, J.A. Mattison, Y. Chen, J. Bacchetta, P. Szulc, R.N. Kitsis, R. De Cabo, R.A. Friedman, C. Torsitano, T.E. McGraw, M. Puchowicz, I. Kurland, G. Karsenty, Osteocalcin Signaling in Myofibers Is Necessary and Sufficient for Optimum Adaptation to Exercise, Cell Metab. 23 (2016) 1078-1092. https://doi.org/10.1016/j.cmet.2016.05.004.

[154] J. Huang, S. Romero-Suarez, N. Lara, C. Mo, S. Kaja, L. Brotto, S.L. Dallas, M.L. Johnson, K. Jähn, L.F. Bonewald, M. Brotto, Crosstalk Between MLO-Y4 Osteocytes and C2C12 Muscle Cells Is Mediated by the Wnt/ $\beta$-Catenin Pathway, JBMR Plus. 1 (2017) 86-100. https://doi.org/10.1002/jbm4.10015.

[155] L.F. Bonewald, M.L. Johnson, Osteocytes, mechanosensing and Wnt signaling, Bone. 42 (2008) 606-615. https://doi.org/10.1016/j.bone.2007.12.224.

[156] J.N. Regan, T. Trivedi, T.A. Guise, D.L. Waning, The Role of TGF $\beta$ in BoneMuscle Crosstalk, Curr. Osteoporos. Rep. 15 (2017) 18-23. https://doi.org/10.1007/s11914-017-0344-5.

[157] D.L. Waning, K.S. Mohammad, S. Reiken, W. Xie, D.C. Andersson, S. John, A. Chiechi, L.E. Wright, A. Umanskaya, M. Niewolna, T. Trivedi, S. Charkhzarrin, P. Khatiwada, A. Wronska, A. Haynes, M.S. Benassi, F.A. Witzmann, G. Zhen, X. Wang, X. Cao, G.D. Roodman, A.R. Marks, T.A. Guise, Excess TGF- $\beta$ mediates muscle weakness associated with bone metastases in mice, Nat. Med. 21 (2015) 1262-1271. https://doi.org/10.1038/nm.3961. 
[158] G. Bin Li, L. Zhang, D.E. Wang, L. AIQudsy, J.X. Jiang, H.Y. Xu, P. Shang, Muscle-bone crosstalk and potential therapies for sarco-osteoporosis, J. Cell. Biochem. 120 (2019) 14262-14273. https://doi.org/10.1002/jcb.28946.

[159] D.B. Maurel, K. Jähn, N. Lara-Castillo, biomedicines Muscle-Bone Crosstalk: Emerging Opportunities for Novel Therapeutic Approaches to Treat Musculoskeletal Pathologies, (n.d.). https://doi.org/10.3390/biomedicines5040062.

[160] J. Rodriguez, B. Vernus, I. Chelh, I. Cassar-Malek, J.C. Gabillard, A. Hadj Sassi, I. Seiliez, B. Picard, A. Bonnieu, Myostatin and the skeletal muscle atrophy and hypertrophy signaling pathways, Cell. Mol. Life Sci. 71 (2014) 4361-4371. https://doi.org/10.1007/s00018-014-1689-x.

[161] A.K. Oestreich, S.M. Carleton, X. Yao, B.A. Gentry, C.E. Raw, M. Brown, F.M. Pfeiffer, Y. Wang, C.L. Phillips, Myostatin deficiency partially rescues the bone phenotype of osteogenesis imperfecta model mice, Osteoporos. Int. 27 (2016) 161-170. https://doi.org/10.1007/s00198-015-3226-7.

[162] Y. Jeong, S.A. Daghlas, Y. Xie, M.A. Hulbert, F.M. Pfeiffer, M.R. Dallas, C.L. Omosule, R.S. Pearsall, S.L. Dallas, C.L. Phillips, Skeletal Response to Soluble Activin Receptor Type IIB in Mouse Models of Osteogenesis Imperfecta, J. Bone Miner. Res. 0 (2018). https://doi.org/10.1002/jbmr.3473.

[163] Y. Jeong, S.A. Daghlas, A.S. Kahveci, D. Salamango, B.A. Gentry, M. Brown, R.S. Rector, R.S. Pearsall, C.L. Phillips, Soluble activin receptor type IIB decoy receptor differentially impacts murine osteogenesis imperfecta muscle function, Muscle Nerve. 57 (2017) 294-304. https://doi.org/10.1002/mus.25706. 
[164] T. Cundy, A. Horne, M. Bolland, G. Gamble, J. Davidson, Bone formation markers in adults with mild osteogenesis imperfecta, Clin. Chem. 53 (2007) 11091114. https://doi.org/10.1373/clinchem.2006.083055.

[165] P. Garnero, A.M. Schott, D. Prockop, G. Chevrel, Bone turnover and type I collagen C-telopeptide isomerization in adult osteogenesis imperfecta: Associations with collagen gene mutations, Bone. 44 (2009) 461-466. https://doi.org/10.1016/j.bone.2008.11.006.

[166] I. Grafe, T. Yang, S. Alexander, E.P. Homan, C. Lietman, M.M. Jiang, T. Bertin, E. Munivez, Y. Chen, B. Dawson, Y. Ishikawa, M.A. Weis, T.K. Sampath, C. Ambrose, D. Eyre, H.P. Bächinger, B. Lee, Excessive transforming growth factor$\beta 2$ signaling is a common mechanism in osteogenesis imperfecta, Nat. Med. 20 (2014) 670-675. https://doi.org/10.1038/nm.3544.

[167] B.P. Sinder, L.E. White, J.D. Salemi, M.S. Ominsky, M.S. Caird, J.C. Marini, K.M. Kozloff, Adult Brtl/+ mouse model of osteogenesis imperfecta demonstrates anabolic response to sclerostin antibody treatment with increased bone mass and strength, Osteoporos. Int. 25 (2014) 2097-2107. https://doi.org/10.1007/s00198014-2737-y.

[168] A. Roschger, P. Roschger, P. Keplingter, K. Klaushofer, S. Abdullah, M. Kneissel, F. Rauch, Effect of sclerostin antibody treatment in a mouse model of severe osteogenesis imperfecta, Bone. 66 (2014) 182-188. https://doi.org/10.1016/j.bone.2014.06.015.

[169] I. Grafe, S. Alexander, T. Yang, C. Lietman, E.P. Homan, E. Munivez, Y. Chen, 
M.M. Jiang, T. Bertin, B. Dawson, F. Asuncion, H.Z. Ke, M.S. Ominsky, B. Lee, Sclerostin Antibody Treatment Improves the Bone Phenotype of Crtap ${ }^{-1}$ Mice, a Model of Recessive Osteogenesis Imperfecta, J. Bone Miner. Res. 31 (2016) 1030-1040. https://doi.org/10.1002/jbmr.2776.

[170] L. Nicol, Y. Wang, R. Smith, J. Sloan, S.C. Nagamani, J. Shapiro, B. Lee, E. Orwoll, Serum Sclerostin Levels in Adults With Osteogenesis Imperfecta: Comparison With Normal Individuals and Response to Teriparatide Therapy, J. Bone Miner. Res. 33 (2018) 307-315. https://doi.org/10.1002/jbmr.3312.

[171] F.H. Glorieux, J.-P. Devogelaer, M. Durigova, S. Goemaere, S. Hemsley, F. Jakob, U. Junker, J. Ruckle, L. Seefried, P.J. Winkle, BPS804 Anti-Sclerostin Antibody in Adults With Moderate Osteogenesis Imperfecta: Results of a Randomized Phase 2a Trial, J. Bone Miner. Res. 32 (2017) 1496-1504. https://doi.org/10.1002/jbmr.3143.

[172] R.J.S. Dhillon, S. Hasni, Pathogenesis and Management of Sarcopenia, Clin. Geriatr. Med. 33 (2017) 17-26. https://doi.org/10.1016/j.cger.2016.08.002.

[173] P. Mary, L. Servais, R. Vialle, Neuromuscular diseases: Diagnosis and management, Orthop. Traumatol. Surg. Res. 104 (2018) S89-S95. https://doi.org/10.1016/j.otsr.2017.04.019.

[174] D.P. Milgrom, N.L. Lad, L.G. Koniaris, T.A. Zimmers, Bone Pain and Muscle Weakness in Cancer Patients, Curr. Osteoporos. Rep. 15 (2017) 76-87. https://doi.org/10.1007/s11914-017-0354-3. 
[175] M. Tabebordbar, E.T. Wang, A.J. Wagers, Skeletal muscle degenerative diseases and strategies for therapeutic muscle repair, Annu. Rev. Pathol. Mech. Dis. 8 (2013) 441-475. https://doi.org/10.1146/annurev-pathol-011811-132450.

[176] A. Heydemann, Skeletal muscle metabolism in duchenne and becker muscular dystrophy—implications for therapies, Nutrients. 10 (2018). https://doi.org/10.3390/nu10060796.

[177] N. Deconinck, B. Dan, Pathophysiology of Duchenne Muscular Dystrophy: Current Hypotheses, Pediatr. Neurol. 36 (2007) 1-7. https://doi.org/10.1016/j.pediatrneurol.2006.09.016.

[178] E.M. Yiu, A.J. Kornberg, Duchenne muscular dystrophy, J. Paediatr. Child Health. 51 (2015) 759-764. https://doi.org/10.1111/jpc.12868.

[179] T. Kawakita, M. Fries, J. Singh, H.B. Al-Kouatly, Pregnancies complicated by maternal osteogenesis imperfecta type III: a case report and review of literature, Clin. Case Reports. 6 (2018) 1252-1257. https://doi.org/10.1002/ccr3.1549.

[180] C.G. Bönnemann, The collagen VI-related myopathies: Ullrich congenital muscular dystrophy and Bethlem myopathy, (n.d.). https://doi.org/10.1016/B9780-08-045031-5.00005-0.

[181] W.A. Irwin, N. Bergamin, P. Sabatelli, C. Reggiani, A. Megighian, L. Merlini, P. Braghetta, M. Columbaro, D. Volpin, G.M. Bressan, P. Bernardi, P. Bonaldo, Mitochondrial dysfunction and apoptosis in myopathic mice with collagen VI deficiency, Nat. Genet. 35 (2003) 367. 
https://doi.org/10.1038/ng1270https://www.nature.com/articles/ng1270\#suppleme ntary-information.

[182] M.T. Lin, M.F. Beal, Mitochondrial dysfunction and oxidative stress in neurodegenerative diseases, Nat. 2006 4437113. 443 (2006) 787-795. https://doi.org/10.1038/nature05292.

[183] E. Marzetti, R. Calvani, M. Cesari, T.W. Buford, M. Lorenzi, B.J. Behnke, C. Leeuwenburgh, Mitochondrial dysfunction and sarcopenia of aging: From signaling pathways to clinical trials, Int. J. Biochem. Cell Biol. 45 (2013) 22882301. https://doi.org/10.1016/j.biocel.2013.06.024.

[184] L. Tilokani, S. Nagashima, V. Paupe, J. Prudent, Mitochondrial dynamics: Overview of molecular mechanisms, Essays Biochem. 62 (2018) 341-360. https://doi.org/10.1042/EBC20170104.

[185] A.M. Van Der Bliek, M.M. Sedensky, P.G. Morgan, Cell biology of the mitochondrion, Genetics. 207 (2017) 843-871. https://doi.org/10.1534/genetics.117.300262.

[186] D.A. Hood, J.M. Memme, A.N. Oliveira, M. Triolo, Maintenance of Skeletal Muscle Mitochondria in Health, Exercise, and Aging, Annu. Rev. Physiol. 81 (2019) 19-41. https://doi.org/10.1146/annurev-physiol-020518-114310.

[187] D.A. Hood, L.D. Tryon, H.N. Carter, Y. Kim, C.C.W. Chen, Unravelling the mechanisms regulating muscle mitochondrial biogenesis, Biochem. J. 473 (2016) 2295. http://www.biochemj.org/content/473/15/2295.abstract. 
[188] T. Wai, T. Langer, Mitochondrial Dynamics and Metabolic Regulation, Trends Endocrinol. Metab. 27 (2016) 105-117. https://doi.org/10.1016/j.tem.2015.12.001.

[189] A.E. Vincent, K. White, T. Davey, J. Philips, R.T. Ogden, C. Lawess, C. Warren, M.G. Hall, Y.S. Ng, G. Falkous, T. Holden, D. Deehan, R.W. Taylor, D.M. Turnbull, M. Picard, Quantitative 3D Mapping of the Human Skeletal Muscle Mitochondrial Network, Cell Rep. 26 (2019) 996-1009.e4. https://doi.org/10.1016/j.celrep.2019.01.010.

[190] F.R. Jornayvaz, G.I. Shulman, Regulation of mitochondrial biogenesis, Essays Biochem. 47 (2010) 69-84. https://doi.org/10.1042/BSE0470069.

[191] L. Wang, G. Lu, H.M. Shen, The Long and the Short of PTEN in the Regulation of Mitophagy, Front. Cell Dev. Biol. 8 (2020) 299. https://doi.org/10.3389/fcell.2020.00299.

[192] A.M. Van Der Bliek, M.M. Sedensky, P.G. Morgan, Cell biology of the mitochondrion, Genetics. 207 (2017) 843-871. https://doi.org/10.1534/genetics.117.300262.

[193] T. Pathak, M. Trebak, Mitochondrial Ca2+ signaling, Pharmacol. Ther. 192 (2018) 112-123. https://doi.org/10.1016/j.pharmthera.2018.07.001.

[194] A. Díaz-Vegas, V. Eisner, E. Jaimovich, Skeletal muscle excitation-metabolism coupling, Arch. Biochem. Biophys. 664 (2019) 89-94. https://doi.org/https://doi.org/10.1016/j.abb.2019.01.037.

[195] S.R. Lamandé, J.F. Bateman, Collagen VI disorders: Insights on form and function 
in the extracellular matrix and beyond, Matrix Biol. 71-72 (2018) 348-367. https://doi.org/https://doi.org/10.1016/j.matbio.2017.12.008.

[196] P. Bernardi, F. Di Lisa, The mitochondrial permeability transition pore: Molecular nature and role as a target in cardioprotection, J. Mol. Cell. Cardiol. 78 (2015) 100-106. https://doi.org/10.1016/j.yjmcc.2014.09.023.

[197] C.P. Baines, M. Gutiérrez-Aguilar, The still uncertain identity of the channelforming unit(s) of the mitochondrial permeability transition pore, Cell Calcium. 73 (2018) 121-130. https://doi.org/https://doi.org/10.1016/j.ceca.2018.05.003.

[198] A.P. Halestrap, What is the mitochondrial permeability transition pore?, J. Mol. Cell. Cardiol. 46 (2009) 821-831. https://doi.org/10.1016/j.yjmcc.2009.02.021.

[199] L. Merlini, A. Angelin, T. Tiepolo, P. Braghetta, P. Sabatelli, A. Zamparelli, A. Ferlini, N.M. Maraldi, P. Bonaldo, P. Bernardi, Cyclosporin A corrects mitochondrial dysfunction and muscle apoptosis in patients with collagen VI myopathies, Proc. Natl. Acad. Sci. U. S. A. 105 (2008) 5225-5229. https://doi.org/10.1073/pnas.0800962105.

[200] L. Merlini, P. Sabatelli, A. Armaroli, S. Gnudi, A. Angelin, P. Grumati, M.E. Michelini, A. Franchella, F. Gualandi, E. Bertini, N.M. Maraldi, A. Ferlini, P. Bonaldo, P. Bernardi, Cyclosporine a in Ullrich congenital muscular dystrophy: Long-term results, Oxid. Med. Cell. Longev. (2011). https://doi.org/10.1155/2011/139194.

[201] D. Hicks, A.K. Lampe, S.H. Laval, V. Allamand, C. Jimenez-Mallebrera, M.C. 
Walter, F. Muntoni, S. Quijano-Roy, P. Richard, V. Straub, H. Lochmüller, K.M.D. Bushby, Cyclosporine A treatment for Ullrich congenital muscular dystrophy: A cellular study of mitochondrial dysfunction and its rescue, Brain. 132 (2009) 147-155. https://doi.org/10.1093/brain/awn289.

[202] L. Biasutto, M. Azzolini, I. Szabò, M. Zoratti, The mitochondrial permeability transition pore in AD 2016: An update, Biochim. Biophys. Acta - Mol. Cell Res. 1863 (2016) 2515-2530. https://doi.org/10.1016/j.bbamcr.2016.02.012.

[203] P. Bonaldo, V. Russo, F. Bucciotti, R. Doliana, A. Colombatti, Structural and functional features of the .alpha.3 chain indicate a bridging role for chicken collagen VI in connective tissues, Biochemistry. 29 (1990) 1245-1254. https://doi.org/10.1021/bi00457a021.

[204] R. Csapo, M. Gumpenberger, B. Wessner, Skeletal Muscle Extracellular Matrix What Do We Know About Its Composition, Regulation, and Physiological Roles? A Narrative Review, Front. Physiol. 11 (2020) 253. https://doi.org/10.3389/fphys.2020.00253.

[205] E. Passerieux, R. Rossignol, A. Chopard, A. Carnino, J.F. Marini, T. Letellier, J.P. Delage, Structural organization of the perimysium in bovine skeletal muscle: Junctional plates and associated intracellular subdomains, J. Struct. Biol. 154 (2006) 206-216. https://doi.org/https://doi.org/10.1016/j.jsb.2006.01.002.

[206] L. Nicol, P. Srikanth, K. Henriksen, S. Sun, R. Smith, M.A. Karsdal, S.C.S. Nagamani, J. Shapiro, B. Lee, B.Z. Leder, E. Orwoll, Widespread disturbance in extracellular matrix collagen biomarker responses to teriparatide therapy in 
osteogenesis imperfecta, Bone. (2020) 115703.

https://doi.org/10.1016/j.bone.2020.115703.

[207] R.H. Engelbert, C.S. Uiterwaal, W.-J. Gerver, J.-J. van der Net, H.E. Pruijs, P.J. Helders, Osteogenesis imperfecta in childhood: Impairment and disability. a prospective study with 4-year follow-up11No commercial party having a direct financial interest in the results of the research supporting this article has or will confer a benefit on the a, Arch. Phys. Med. Rehabil. 85 (2004) 772-778. https://doi.org/https://doi.org/10.1016/j.apmr.2003.08.085.

[208] O. BAR-OR, Pathophysiological factors which limit the exercise capacity of the sick child, Med. Sci. Sport. Exerc. 18 (1986) 276-282. https://journals.lww.com/acsmmsse/Fulltext/1986/06000/Pathophysiological_factors_which_limit_the.4.aspx.

[209] S. Khosla, B.L. Riggs, Pathophysiology of Age-Related Bone Loss and Osteoporosis, Endocrinol. Metab. Clin. North Am. 34 (2005) 1015-1030. https://doi.org/https://doi.org/10.1016/j.ecl.2005.07.009.

[210] A. Biggin, C.F. Munns, Osteogenesis imperfecta: diagnosis and treatment, Curr Osteoporos Rep. 12 (2014) 279-288. https://doi.org/10.1007/s11914-014-0225-0.

[211] D.A. Bailey, H.A. McKay, R.L. Mirwald, P.R.E. Crocker, R.A. Faulkner, A SixYear Longitudinal Study of the Relationship of Physical Activity to Bone Mineral Accrual in Growing Children: The University of Saskatchewan Bone Mineral Accrual Study, J. Bone Miner. Res. 14 (2009) 1672-1679. https://doi.org/10.1359/jbmr.1999.14.10.1672. 
[212] F. Detter, B.E. Rosengren, M. Dencker, M. Lorentzon, J.-Å. Nilsson, M.K. Karlsson, A 6-Year Exercise Program Improves Skeletal Traits Without Affecting Fracture Risk: A Prospective Controlled Study in 2621 Children, J. Bone Miner. Res. 29 (2014) 1325-1336. https://doi.org/10.1002/jbmr.2168.

[213] Y. Hagihara, A. Nakajima, S. Fukuda, S. Goto, H. Iida, M. Yamazaki, Running exercise for short duration increases bone mineral density of loaded long bones in young growing rats, Tohoku J. Exp. Med. 219 (2009) 139-143. https://doi.org/10.1620/tjem.219.139.

[214] H. Isaksson, V. Tolvanen, M.A.J. Finnilä, J. Iivarinen, J. Tuukkanen, K. Seppänen, J.P.A. Arokoski, P.A. Brama, J.S. Jurvelin, H.J. Helminen, Physical Exercise Improves Properties of Bone and Its Collagen Network in Growing and Maturing Mice, Calcif. Tissue Int. 85 (2009) 247-256. https://doi.org/10.1007/s00223-0099273-3.

[215] J. Iwamoto, J.K. Yeh, J.F. Aloia, Differential effect of treadmill exercise on three cancellous bone sites in the young growing rat, Bone. 24 (1999) 163-169. https://doi.org/https://doi.org/10.1016/S8756-3282(98)00189-6.

[216] J. Iwamoto, T. Takeda, S. Ichimura, Effects of Exercise on Bone Mineral Density in Mature Osteopenic Rats, J. Bone Miner. Res. 13 (2009) 1308-1317. https://doi.org/10.1359/jbmr.1998.13.8.1308.

[217] J. Iwamoto, T. Takeda, S. Ichimura, Effect of exercise on tibial and lumbar vertebral bone mass in mature osteopenic rats: bone histomorphometry study, J. Orthop. Sci. 3 (1998) 257-263. https://doi.org/10.1007/s007760050051. 
[218] T.H. Huang, S.C. Lin, F.L. Chang, S.S. Hsieh, S.H. Liu, R.S. Yang, Effects of different exercise modes on mineralization, structure, and biomechanical properties of growing bone, J. Appl. Physiol. 95 (2003) 300-307. https://doi.org/10.1152/japplphysiol.01076.2002.

[219] M.J. Falcai, A. Zamarioli, G.B. Leoni, M.D. de Sousa Neto, J.B. Volpon, Swimming Activity Prevents the Unloading Induced Loss of Bone Mass, Architecture, and Strength in Rats, Biomed Res. Int. 2015 (2015) 507848. https://doi.org/10.1155/2015/507848.

[220] Y.-I. Ju, T. Sone, K. Ohnaru, K. Tanaka, M. Fukunaga, Effect of swimming exercise on three-dimensional trabecular bone microarchitecture in ovariectomized rats, J. Appl. Physiol. 119 (2015) 990-997. https://doi.org/10.1152/japplphysiol.00147.2015.

[221] J.B. Volpon, A.V. Silva, M.J. Falcai, M.J.Q. Louzada, A. Zamarioli, B.G.D.S. Kotake, J.P.M. Issa, The osteogenic effects of swimming on bone mass, strength, and microarchitecture in rats with unloading-induced bone loss, Microsc. Res. Tech. 78 (2015) 784-791. https://doi.org/10.1002/jemt.22541.

[222] W.H. Hoshi A Chiba M, Inaba Y, Bone density and mechanical properties in femoral bone of swim loaded aged mice, Biomed Enviorn Sci. 11 (1998) 243-250.

[223] F.T.T. Frajacomo, M.J. Falcai, C.R. Fernandes, A.C. Shimano, S.B. Garcia, Biomechanical adaptations of mice cortical bone submitted to three different exercise modalities, Acta Ortop. Bras. 21 (2013) 328-332. https://doi.org/10.1590/S1413-78522013000600006. 
[224] S.M. Carleton, D.J. McBride, W.L. Carson, C.E. Huntington, K.L. Twenter, K.M. Rolwes, C.T. Winkelmann, J.S. Morris, J.F. Taylor, C.L. Phillips, Role of Genetic Background in Determining Phenotypic Severity Throughout Postnatal Development and at Peak Bone Mass in Col1a2 Deficient Mice (oim), Bone. 42 (2008) 681-694. https://doi.org/10.1016/j.bone.2007.12.215.

[225] M. Brown, J. Ning, J.A. Ferreira, J.L. Bogener, D.B. Lubahn, Estrogen receptor- $\alpha$ and $-\beta$ and aromatase knockout effects on lower limb muscle mass and contractile function in female mice, Am. J. Physiol. Metab. 296 (2009) E854-E861. https://doi.org/10.1152/ajpendo.90696.2008.

[226] R.S. Rector, J.P. Thyfault, R.T. Morris, M.J. Laye, S.J. Borengasser, F.W. Booth, J.A. Ibdah, Daily exercise increases hepatic fatty acid oxidation and prevents steatosis in Otsuka Long-Evans Tokushima Fatty rats, Am. J. Physiol. Gastrointest. Liver Physiol. 294 (2008). https://doi.org/10.1152/ajpgi.00428.2007.

[227] J.A. Fletcher, G.M. Meers, M.A. Linden, M.L. Kearney, E.M. Morris, J.P. Thyfault, R.S. Rector, Impact of Various Exercise Modalities on Hepatic Mitochondrial Function, Med. Sci. Sports Exerc. 46 (2014) 1089-1097. https://doi.org/10.1249/MSS.0000000000000223.

[228] E. Matthew Morris, G.M.E. Meers, F.W. Booth, K.L. Fritsche, C.D. Hardin, J.P. Thyfault, J.A. Ibdah, Pgc-1 $\alpha$ overexpression results in increased hepatic fatty acid oxidation with reduced triacylglycerol accumulation and secretion, Am. J. Physiol. - Gastrointest. Liver Physiol. 303 (2012). https://doi.org/10.1152/ajpgi.00169.2012. 
[229] A. Ruiz-Ramírez, M. Chávez-Salgado, J.A. Peñeda-Flores, E. Zapata, F. Masso, M. El-Hafidi, High-sucrose diet increases ROS generation, FFA accumulation, UCP2 level, and proton leak in liver mitochondria, Am. J. Physiol. Metab. 301 (2011) E1198-E1207. https://doi.org/10.1152/ajpendo.00631.2010.

[230] H.J.G. Gundersen, T.F. Bendtsen, L. Korbo, N. Marcussen, A. MØLler, K. Nielsen, J.R. Nyengaard, B. Pakkenberg, F.B. SØRensen, A. Vesterby, M.J. West, Some new, simple and efficient stereological methods and their use in pathological research and diagnosis, APMIS. 96 (1988) 379-394. https://doi.org/10.1111/j.1699-0463.1988.tb05320.x.

[231] F.G.S. Toledo, S. Watkins, D.E. Kelley, Changes Induced by Physical Activity and Weight Loss in the Morphology of Intermyofibrillar Mitochondria in Obese Men and Women, J. Clin. Endocrinol. Metab. 91 (2006) 3224-3227. https://doi.org/10.1210/jc.2006-0002.

[232] A.N. Malik, A. Czajka, P. Cunningham, Accurate quantification of mouse mitochondrial DNA without co-amplification of nuclear mitochondrial insertion sequences, Mitochondrion. 29 (2016) 59-64. https://doi.org/https://doi.org/10.1016/j.mito.2016.05.003.

[233] P.A. Srere, [1] Citrate synthase: [EC 4.1.3.7. Citrate oxaloacetate-lyase (CoAacetylating)], in: Methods Enzymol., Academic Press, 1969: pp. 3-11. https://doi.org/https://doi.org/10.1016/0076-6879(69)13005-0.

[234] W.G. Aschenbach, M.F. Hirshman, N. Fujii, K. Sakamoto, K.F. Howlett, L.J. Goodyear, Effect of AICAR Treatment on Glycogen Metabolism in Skeletal 
Muscle, Diabetes. 51 (2002) 567.

http://diabetes.diabetesjournals.org/content/51/3/567.abstract.

[235] S.G. Carmer, M.R. Swanson, An Evaluation of Ten Pairwise Multiple Comparison Procedures by Monte Carlo Methods, J. Am. Stat. Assoc. 68 (1973) 66-74. https://doi.org/10.1080/01621459.1973.10481335.

[236] D.J. Saville, Multiple Comparison Procedures: The Practical Solution, Am. Stat. 44 (1990) 174-180. https://doi.org/10.1080/00031305.1990.10475712.

[237] W.J. Conover, R.L. Iman, Analysis of Covariance Using the Rank Transformation, Biometrics. 38 (1982) 715-724. https://doi.org/10.2307/2530051.

[238] I.R. Lanza, K.S. Nair, Functional Assessment of Isolated Mitochondria In Vitro, Methods Enzymol. 457 (2009) 349-372. https://doi.org/10.1016/S00766879(09)05020-4.

[239] S. Larsen, J. Nielsen, C.N. Hansen, L.B. Nielsen, F. Wibrand, N. Stride, H.D. Schroder, R. Boushel, J.W. Helge, F. Dela, M. Hey-Mogensen, Biomarkers of mitochondrial content in skeletal muscle of healthy young human subjects, J. Physiol. 590 (2012) 3349-3360. https://doi.org/10.1113/jphysiol.2012.230185.

[240] A. Vigels $\varnothing$, N.B. Andersen, F. Dela, The relationship between skeletal muscle mitochondrial citrate synthase activity and whole body oxygen uptake adaptations in response to exercise training, Int. J. Physiol. Pathophysiol. Pharmacol. 6 (2014) 84-101. http://www.ncbi.nlm.nih.gov/pmc/articles/PMC4106645/.

[241] H.N. Carter, Y. Kim, A.T. Erlich, D. Zarrin-khat, D.A. Hood, Autophagy and 
mitophagy flux in young and aged skeletal muscle following chronic contractile activity, J. Physiol. 596 (2018) 3567-3584. https://doi.org/10.1113/JP275998.

[242] E. Tasdemir, L. Galluzzi, M.C. Maiuri, A. Criollo, I. Vitale, E. Hangen, N. Modjtahedi, G. Kroemer, Methods for Assessing Autophagy and Autophagic Cell Death, in: V. Deretic (Ed.), Autophagosome and Phagosome, Humana Press, Totowa, NJ, 2008: pp. 29-76. https://doi.org/10.1007/978-1-59745-157-4_3.

[243] K.C. Kregel, D.L. Allen, F.W. Booth, M.R. Fleshner, E.J. Henriksen, T.I. Musch, D.S. O’Leary, C.M. Parks, D.C. Poole, A.W. Ra'anan, D.D. Sheriff, M.S. Sturek, L.A. Toth, Resource Book for the Design of Animal Excercise Protocols, American Physiological Society , 2006. http://www.theaps.org/mm/SciencePolicy/AnimalResearch/Publications/Animal-ExerciseProtocols/book14824.pdf.

[244] I.H. Thomas, L.A. DiMeglio, Advances in the Classification and Treatment of Osteogenesis Imperfecta, Curr. Osteoporos. Rep. 14 (2016) 1-9. https://doi.org/10.1007/s11914-016-0299-y.

[245] X. Yao, S.M. Carleton, A.D. Kettle, J. Melander, C.L. Phillips, Y. Wang, Genderdependence of bone structure and properties in adult osteogenesis imperfecta murine model, Ann. Biomed. Eng. 41 (2013) 1139-1149. https://doi.org/10.1007/s10439-013-0793-7.

[246] C.H. Turner, A.G. Robling, Designing Exercise Regimens to Increase Bone Strength, Exerc. Sport Sci. Rev. 31 (2003) 45-50. https://journals.lww.com/acsmessr/Fulltext/2003/01000/Designing_Exercise_Regimens_to_Increase_Bone.9.asp 
$\mathrm{X}$.

[247] N.H. Hart, S. Nimphius, T. Rantalainen, A. Ireland, A. Siafarikas, R.U. Newton, Mechanical basis of bone strength: influence of bone material, bone structure and muscle action, J. Musculoskelet. Neuronal Interact. 17 (2017) 114-139. https://www.ncbi.nlm.nih.gov/pubmed/28860414.

[248] K. Khan, H.A. McKay, H. Haapasalo, K.L. Bennell, M.R. Forwood, P. Kannus, J.D. Wark, Does childhood and adolescence provide a unique opportunity for exercise to strengthen the skeleton?, J. Sci. Med. Sport. 3 (2000) 150-164. https://doi.org/https://doi.org/10.1016/S1440-2440(00)80077-8.

[249] A.G. Robling, F.M. Hinant, D.B. Burr, C.H. Turner, Improved Bone Structure and Strength After Long-Term Mechanical Loading Is Greatest if Loading Is Separated Into Short Bouts, J. Bone Miner. Res. 17 (2009) 1545-1554. https://doi.org/10.1359/jbmr.2002.17.8.1545.

[250] W. Kühlbrandt, Structure and function of mitochondrial membrane protein complexes, BMC Biol. 13 (2015) 89. https://doi.org/10.1186/s12915-015-0201-x.

[251] C. Porter, B.T. Wall, Skeletal muscle mitochondrial function: is it quality or quantity that makes the difference in insulin resistance?, J. Physiol. 590 (2012) 5935-5936. https://doi.org/10.1113/jphysiol.2012.241083.

[252] H. Michel, The mechanism of proton pumping by cytochrome c oxidase, Proc. Natl. Acad. Sci. U. S. A. 95 (1998) 12819-12824. http://www.ncbi.nlm.nih.gov/pmc/articles/PMC23610/. 
[253] A. Turrina, M.A. Martínez-González, C. Stecco, The muscular force transmission system: Role of the intramuscular connective tissue, J. Bodyw. Mov. Ther. 17 (2013) 95-102. https://doi.org/https://doi.org/10.1016/j.jbmt.2012.06.001.

[254] E. Passerieux, R. Rossignol, T. Letellier, J.P. Delage, Physical continuity of the perimysium from myofibers to tendons: Involvement in lateral force transmission in skeletal muscle, J. Struct. Biol. 159 (2007) 19-28. https://doi.org/https://doi.org/10.1016/j.jsb.2007.01.022.

[255] E. Sorato, S. Menazza, A. Zulian, P. Sabatelli, F. Gualandi, L. Merlini, P. Bonaldo, M. Canton, P. Bernardi, F. Di Lisa, Monoamine oxidase inhibition prevents mitochondrial dysfunction and apoptosis in myoblasts from patients with collagen VI myopathies, Free Radic. Biol. Med. 75 (2014) 40-47. https://doi.org/https://doi.org/10.1016/j.freeradbiomed.2014.07.006.

[256] B. Mueller, R. Engelbert, F. Baratta-Ziska, B. Bartels, N. Blanc, E. Brizola, P. Fraschini, C. Hill, C. Marr, L. Mills, K. Montpetit, V. Pacey, M.R. Molina, M. Schuuring, C. Verhille, O. de Vries, E.H.K. Yeung, O. Semler, Consensus statement on physical rehabilitation in children and adolescents with osteogenesis imperfecta, Orphanet J. Rare Dis. 13 (2018) 158. https://doi.org/10.1186/s13023018-0905-4.

[257] O. Fricke, E. Schoenau, The 'Functional Muscle-Bone Unit': Probing the relevance of mechanical signals for bone development in children and adolescents, Growth Horm. IGF Res. 17 (2007) 1-9. https://doi.org/https://doi.org/10.1016/j.ghir.2006.10.004. 
[258] V.L. Gremminger, Y. Jeong, R.P. Cunningham, G.M. Meers, R.S. Rector, C.L. Phillips, Compromised Exercise Capacity and Mitochondrial Dysfunction in the Osteogenesis Imperfecta Murine (oim) Mouse Model, J. Bone Miner. Res. 34 (2019). https://doi.org/10.1002/jbmr.3732.

[259] P. Porsborg, G. Astrup, D. Bendixen, A.M. Lund, H. Ørding, Osteogenesis imperfecta and malignant hyperthermia, Anaesthesia. 51 (1996) 863-865. https://doi.org/10.1111/j.1365-2044.1996.tb12619.x.

[260] E.M. Morris, G.M.E. Meers, F.W. Booth, K.L. Fritsche, C.D. Hardin, J.P. Thyfault, J.A. Ibdah, PGC-1 $\alpha$ overexpression results in increased hepatic fatty acid oxidation with reduced triacylglycerol accumulation and secretion, Am. J. Physiol. - Gastrointest. Liver Physiol. 303 (2012) G979-G992. https://doi.org/10.1152/ajpgi.00169.2012.

[261] T.D. Olver, J.C. Edwards, T.J. Jurrissen, A.B. Veteto, J.L. Jones, C. Gao, C. Rau, C.M. Warren, P.J. Klutho, L. Alex, S.C. Ferreira-Nichols, J.R. Ivey, P.K. Thorne, K.S. McDonald, M. Krenz, C.P. Baines, R.J. Solaro, Y. Wang, D.A. Ford, T.L. Domeier, J. Padilla, R.S. Rector, C.A. Emter, Western Diet-Fed, Aortic-Banded Ossabaw Swine: A Preclinical Model of Cardio-Metabolic Heart Failure, JACC Basic to Transl. Sci. 4 (2019) 404-421. https://doi.org/10.1016/j.jacbts.2019.02.004.

[262] R.S. Rector, J.P. Thyfault, R.T. Morris, M.J. Laye, S.J. Borengasser, F.W. Booth, J.A. Ibdah, Daily exercise increases hepatic fatty acid oxidation and prevents steatosis in Otsuka Long-Evans Tokushima Fatty rats, Am. J. Physiol. Liver 
Physiol. 294 (2008) G619-G626. https://doi.org/10.1152/ajpgi.00428.2007.

[263] J.B.D.B. Weir, New methods for calculating metabolic rate with special reference to protein metabolism, J. Physiol. 109 (1949) 1-9. https://www.ncbi.nlm.nih.gov/pubmed/15394301.

[264] E. Jequier, K. Acheson, Y. Schutz, Assessment of Energy Expenditure and Fuel Utilization in Man, Annu. Rev. Nutr. (1987). https://doi.org/10.1146/annurev.nu.07.070187.001155.

[265] A. Mann, A. Thompson, N. Robbins, A.L. Blomkalns, Localization, identification, and excision of murine adipose depots, J. Vis. Exp. (2014). https://doi.org/10.3791/52174.

[266] M. Kammoun, I. Cassar-Malek, B. Meunier, B. Picard, A simplified immunohistochemical classification of skeletal muscle fibres in mouse, Eur. J. Histochem. 58 (2014) 2254. https://doi.org/10.4081/ejh.2014.2254.

[267] V. Augusto, C.R. Padovani, G.E. Rocha Campos, Skeletal muscle fiber types in C57B16J mice, 2004.

[268] T.B. Willingham, K.K. McCully, In vivo assessment of mitochondrial dysfunction in clinical populations using near-infrared spectroscopy, Front. Physiol. 8 (2017) 689. https://doi.org/10.3389/fphys.2017.00689.

[269] K.E. Conley, S.A. Jubrias, P.C. Esselman, Oxidative capacity and ageing in human muscle, J. Physiol. 526 (2000) 203-210. https://doi.org/10.1111/j.14697793.2000.t01-1-00203.x. 
[270] M. Periasamy, J.L. Herrera, F.C.G. Reis, Skeletal muscle thermogenesis and its role in whole body energy metabolism, Diabetes Metab. J. 41 (2017) 327-336. https://doi.org/10.4093/dmj.2017.41.5.327.

[271] B.B. Rasmussen, S.M. Phillips, Contractile and nutritional regulation of human muscle growth, Exerc. Sport Sci. Rev. 31 (2003) 127-131. https://doi.org/10.1097/00003677-200307000-00005.

[272] B.D. Ross, R. Hems, H.A. Krebs, The Rate of Gluconeogenesis from Various Precursors in the Perfused Rat Liver, 1967.

[273] F. Rotondo, A.C. Ho-Palma, X. Remesar, J.A. Fernández-López, M.D.M. Romero, M. Alemany, Glycerol is synthesized and secreted by adipocytes to dispose of excess glucose, via glycerogenesis and increased acyl-glycerol turnover, Sci. Rep. 7 (2017) 1-14. https://doi.org/10.1038/s41598-017-09450-4.

[274] Y. Mahendran, H. Cederberg, J. Vangipurapu, A.J. Kangas, P. Soininen, J. Kuusisto, M. Uusitupa, M. Ala-Korpela, M. Laakso, Glycerol and fatty acids in serum predict the development of hyperglycemia and type 2 diabetes in Finnish men, Diabetes Care. 36 (2013) 3732-3738. https://doi.org/10.2337/dc13-0800.

[275] G. Benard, B. Faustin, E. Passerieux, A. Galinier, C. Rocher, N. Bellance, J.P. Delage, L. Casteilla, T. Letellier, R. Rossignolinserm, Physiological diversity of mitochondrial oxidative phosphorylation, Am. J. Physiol. - Cell Physiol. 291 (2006) 1172-1182. https://doi.org/10.1152/ajpcell.00195.2006.

[276] E. Fernández-Vizarra, J.A. Enríquez, A. Pérez-Martos, J. Montoya, P. Fernández- 
Silva, Tissue-specific differences in mitochondrial activity and biogenesis, Mitochondrion. 11 (2011) 207-213. https://doi.org/10.1016/j.mito.2010.09.011.

[277] D.T. Johnson, R.A. Harris, S. French, P. V. Blair, J. You, K.G. Bemis, M. Wang, R.S. Balaban, Tissue heterogeneity of the mammalian mitochondrial proteome, Am. J. Physiol. - Cell Physiol. 292 (2007) 689-697. https://doi.org/10.1152/ajpcell.00108.2006.

[278] S. Schiaffino, C. Reggiani, Fiber types in Mammalian skeletal muscles, Physiol. Rev. 91 (2011) 1447-1531. https://doi.org/10.1152/physrev.00031.2010.

[279] G.C. Bogdanis, Effects of physical activity and inactivity on muscle fatigue, Front. Physiol. 3 MAY (2012). https://doi.org/10.3389/fphys.2012.00142.

[280] E. V Menshikova, V.B. Ritov, L. Fairfull, R.E. Ferrell, D.E. Kelley, B.H. Goodpaster, Effects of Exercise on Mitochondrial Content and Function in Aging Human Skeletal Muscle, n.d.

[281] W.H. Kunau, V. Dommes, H. Schulz, $\beta$-Oxidation of fatty acids in mitochondria, peroxisomes, and bacteria: A century of continued progress, Prog. Lipid Res. 34 (1995) 267-342. https://doi.org/10.1016/0163-7827(95)00011-9.

[282] L. Folkestad, J.D. Hald, A.K. Ersbøll, J. Gram, A.P. Hermann, B. Langdahl, B. Abrahamsen, K. Brixen, Fracture Rates and Fracture Sites in Patients With Osteogenesis Imperfecta: A Nationwide Register-Based Cohort Study, J. Bone Miner. Res. 32 (2017) 125-134. https://doi.org/10.1002/jbmr.2920.

[283] P. Bonaldo, V. Russo, F. Bucciotti, R. Doliana, A. Colombatti, Structural and 
Functional Features of the $\alpha 3$ Chain Indicate a Bridging Role for Chicken Collagen VI in Connective Tissues, Biochemistry. 29 (1990) 1245-1254. https://doi.org/10.1021/bi00457a021.

[284] W. Chen, M.R. Nyasha, M. Koide, M. Tsuchiya, N. Suzuki, Y. Hagiwara, M. Aoki, M. Kanzaki, In vitro exercise model using contractile human and mouse hybrid myotubes, Sci. Rep. 9 (2019) 1-9. https://doi.org/10.1038/s41598-01948316-9.

[285] N. Biesemann, J.S. Ried, D. Ding-Pfennigdorff, A. Dietrich, C. Rudolph, S. Hahn, W. Hennerici, C. Asbrand, T. Leeuw, C. Strübing, High throughput screening of mitochondrial bioenergetics in human differentiated myotubes identifies novel enhancers of muscle performance in aged mice, Sci. Rep. 8 (2018) 1-12. https://doi.org/10.1038/s41598-018-27614-8.

[286] R. Morello, T.K. Bertin, Y. Chen, J. Hicks, L. Tonachini, M. Monticone, P. Castagnola, F. Rauch, F.H. Glorieux, J. Vranka, H.P. Bächinger, J.M. Pace, U. Schwarze, P.H. Byers, M. Weis, R.J. Fernandes, D.R. Eyre, Z. Yao, B.F. Boyce, B. Lee, CRTAP Is Required for Prolyl 3- Hydroxylation and Mutations Cause Recessive Osteogenesis Imperfecta, Cell. 127 (2006) 291-304. https://doi.org/https://doi.org/10.1016/j.cell.2006.08.039. 


\section{VITA}

Victoria Lynn Gremminger was born January $4^{\text {th }}, 1994$ in St. Louis, MO and is the daughter of Patty Brooks and Brett Grill, step-daughter of Pam Grill and William Brooks, sister of Samantha and Alexandria Grill, wife of Thomas Gremminger and mother of Augustus Gremminger. Victoria first discovered her passion for science while taking biology and chemistry courses at Windsor High School. She decided to attend Missouri University of Science and Technology where she received a Bachelor of Science degree in Biology with a minor in Chemistry. Following her undergraduate studies, Victoria was accepted to the Biochemistry Graduate Program at the University of Missouri to further develop her interests and understanding of Biochemistry. Here, she was fortunate to join the laboratory of Dr. Charlotte L Phillips where she was able to study skeletal muscle weakness and mitochondrial dysfunction in mouse models of osteogenesis imperfecta. Under Dr. Phillips' guidance, Victoria was able to blossom into an independent researcher while further developing her understanding of osteogenesis imperfecta and technical skills. Victoria intends to pursue a career in industry where she can put her knowledge of bone-muscle biology and crosstalk to use developing novel therapies. 